\title{
Experimental Investigation of Visible Diffraction in Tilted Fibre Bragg Gratings
}

\author{
Milad Abou Dakka \\ B.Eng., M.Eng.
}

\begin{abstract}
A thesis submitted to the Faculty of Graduate and Post-Doctoral Affairs in partial fulfillment of the requirements for the degree of Master of Applied Science in Electrical and Computer Engineering
\end{abstract}

\author{
Department of Electronics \\ Carleton University
}

Ottawa-Carleton Institute for Electrical and Computer Engineering

Ottawa, Ontario, Canada

May 2014

(C) Milad Abou Dakka 


\begin{abstract}
We present an analysis on the visible diffraction patterns of Tilted Fiber Bragg Gratings (TFBG), with applications to blue-laser fiber sensors. On the basis of our current understanding of a visible diffraction phenomenon called sidetapping, or outtapping, we compare theoretical predictions with experimental results.

In order to compare theory to experiment we obtain empirical observations of diffraction angles. A $1550 \mathrm{~nm}-\mathrm{TFBG}$ is connected to a Coherent Spectrum-70C laser source and measurements are taken for several wavelengths including red $(647.1 \mathrm{~nm})$, yellow $(568.2 \mathrm{~nm})$, green $(514.5 \mathrm{~nm})$, blue $(488.0 \mathrm{~nm})$ and violet $(457.9 \mathrm{~nm})$ to show that our results are in definite agreement with theory. There are other variables that affect and govern this diffraction behaviour so a comprehensive parameter study is used to relate the input variables to output variables. Output variables are power and longitudinal angles of each radiating diffraction order. Input variables would include the type of fiber used, grating pitch, grating tilt, and wavelength. With information gathered from the input-output analysis, we continue by studying blue light diffraction. Different devices are compared to gain insight into the optimal conditions for a blue light TFBG sensor. The diffraction of blue light in particular is exploited to gain insight on specialized fiber nano-coatings used in biochemical sensors.

To start we review the existing methods of TFBG diffraction in the visible spectrum, including Coupled Mode analysis and a brief overview of alternate methods. Generally, depending on the wavelength and other factors there may or may not be coupling to radiation modes. The specific angles of these radiation modes with respect to the fiber axis, called longitudinal angles, are determined by theoretical phase-matching conditions related to the incoming beam wavelength.
\end{abstract}




\section{Acknowledgements}

My immediate thanks go to Dr. Jacques Albert and Dr. Christopher Smelser, my thesis supervisors, for their constant support, encouragement, and professional demeanour throughout my thesis research. You were both always ready to provide invaluable suggestions and to conduct productive discussions. Our lab technician, Albane Laronche was integral to the realization of this project. She was always available for troubleshooting and to help with laboratory equipment and procedures. Ultimately, your professionalism and insights were a major factor in the success of this research and your contribution is duly appreciated.

A special thanks goes to Dr. Anatoli lanoul, Daniel Prezgot and Adam Bottomley of Carleton University's Department of Chemistry for providing the workspace required for the completion of this project and helping me in ways I can only describe as selfless and adept.

Similarly, I am infinitely grateful to Penka Matanska and Mike Antunes of the Physics Department for lending a Goniometer, which turned out to be the key to the success of this research.

Finally, none of this work was possible without the Department of Electronics, from its Faculty to its office administrators. Namely, Blazenka Power, Anna Lee and Sylvie Beekmans together form a real dream-team and their readiness to help with matters great and small, as well as the friendly atmosphere they radiate, will be sorely missed.

I will thank my dear lab mates Zhang Yang, Sandrine Lepinay, Alex Beliaev, Wenjun Zhou, Katherine Bock, Changyu Shen, Ruichao Li and Lingyun Xiong. It was certainly a great experience working alongside all of them.

I would also like to thank my friends and colleagues. I should especially thank those who helped organize my material and provided precious feedback.

Last but certainly not least, I thank my wonderful family, for their unconditional love.

Ottawa, 2014 Milad Dakka 
To Marina

"Into her sea, in warmth and solace,

I am reborn." 


\section{Table of Contents}

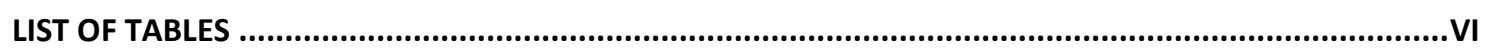

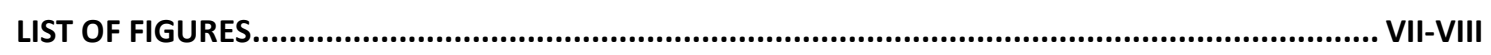

LIST OF NOTATIONS AND ABBREVIATIONS .............................................................................................

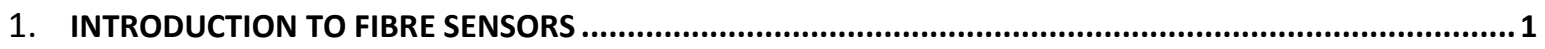

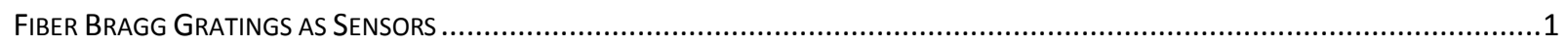

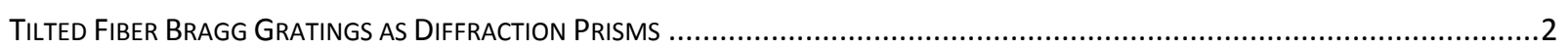

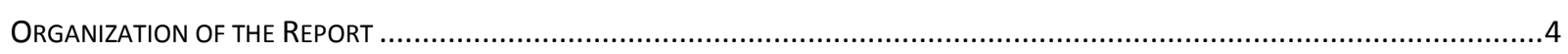

2. TILTED FIBRE BRAGG GRATINGS IN THE VISIBLE SPECTRUM ...................................................... 6

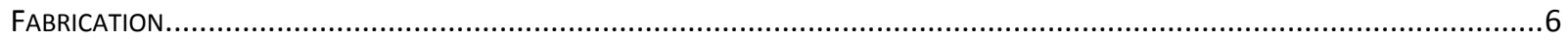

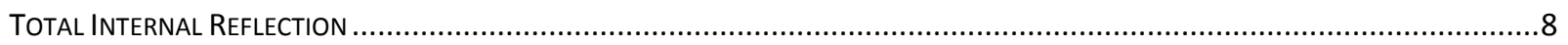

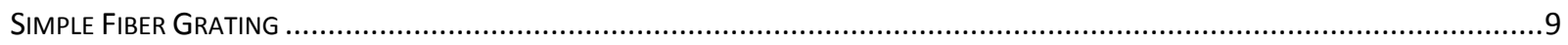

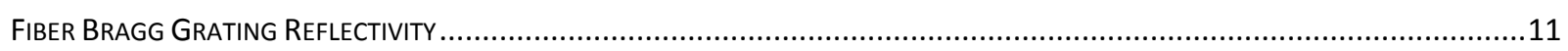

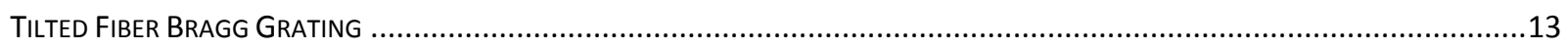

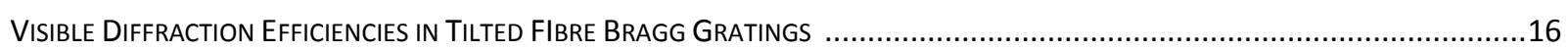

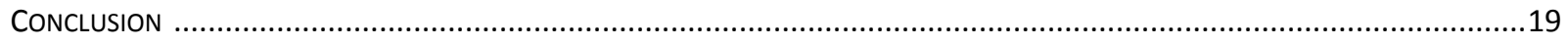

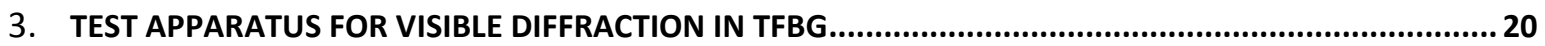

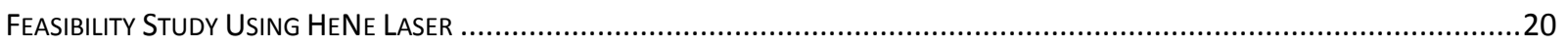

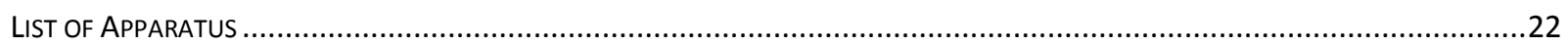

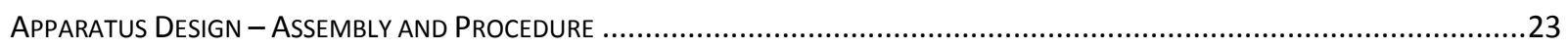

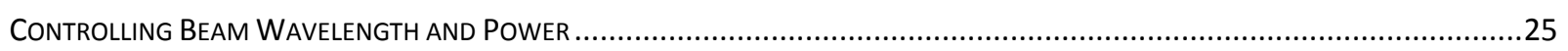

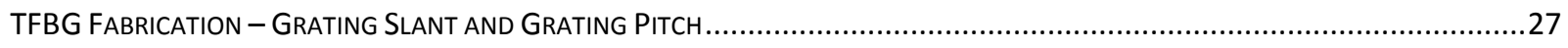

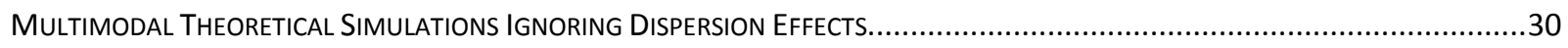

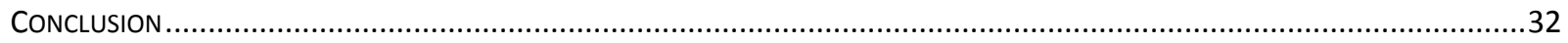

4. RESULTS OF VISIBLE DIFFRACTION EXPERIMENTS: ANGULAR AND POWER DISTRIBUTION................... 34

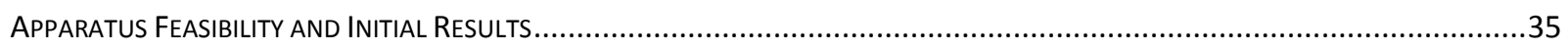

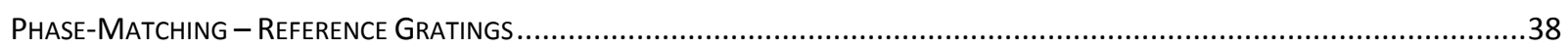

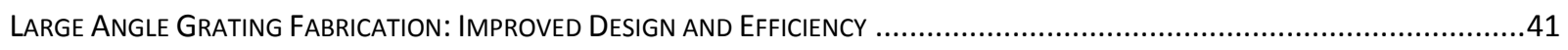

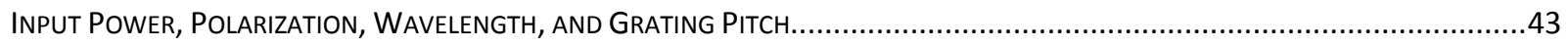

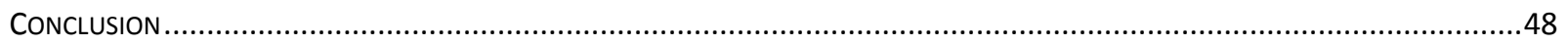

5. ANALYSIS AND SIGNIFICANCE OF ANGULAR AND POWER DISTRIBUTION RESULTS ...........................49

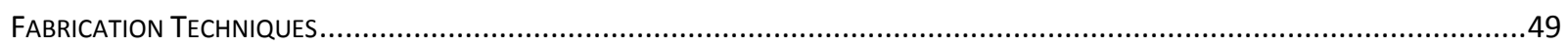

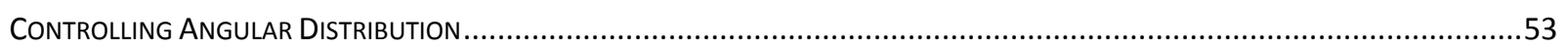

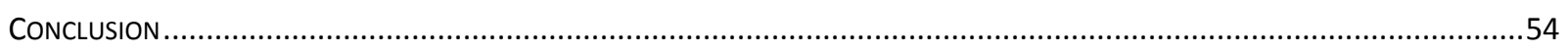

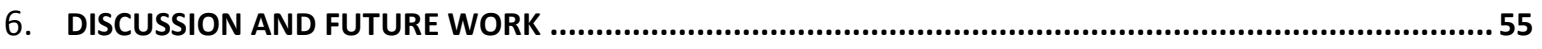

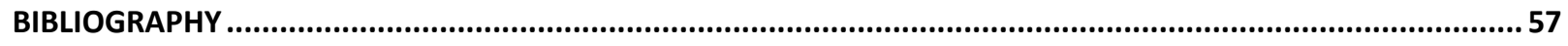

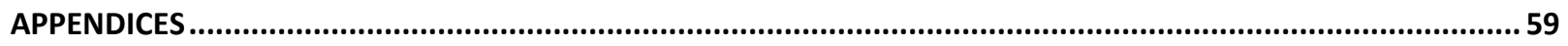




\section{List of Tables}

4.1.1 Types of gratings tested in this research, fabricated out-of-lab and in-lab.

4.3.1 The angles of radiation for four modes, and their respective efficiencies, are given for three gratings.

4.4.1 The angles of radiation for four modes, and their respective efficiencies for sample grating $\left(37.5^{\circ}\right)$.

4.4.2 The angles of radiation, experimental and theoretical, for the backwardsidetapped mode for various wavelengths.

5.2.1 Theoretical exit angles for blue light radiation for $1610 \mathrm{~nm}, 976 \mathrm{~nm}$ and $663 \mathrm{~nm}$ TFBGs. 


\section{List of Figures}

1.2.1 A white-light source hitting a theoretical 1550nm FBG tilted by $37.5^{\circ}$.

2.2.1 Vector diagram highlights the difference between refraction and TIR.

2.3.1 A simple grating. Incident, reflected and grating wavevectors are shown. $\quad$..............10

2.4.1 Sample reflection spectrum for FBG versus detuning from center wavelength. $\quad$...............12

2.5.1 The effective grating periodicity remains unchanged along the fiber axis.

2.5.2 A typical TFBG transmission spectrum showing higher order mode losses.

2.5.3 Phase matching analysis of incoming $\boldsymbol{k}_{i}$, grating $\boldsymbol{k}_{G}$ and radiation $\boldsymbol{k}_{R}$ wavevectors. ..............14

2.6.1 Scattering from the fibre core with the FBG. Light scatters in cone shape. $\quad$...............17

3.1.1 Testing the feasibility of visible light diffraction in standard SMF-28 fibre. $\quad$..............21

3.1.2 A sample of a radiation maximum observed at a particular angle $\theta_{\mathrm{m}} . \quad \ldots \ldots \ldots \ldots . .21$

3.2.1 Schematic of apparatus setup. $\quad$...............22

3.3.1 Apparatus design and breakdown. $\quad$...............23

3.3.2 Apparatus operation. $\quad$...............24

3.4.1 Both power and wavelength can be finely tuned. $\quad$................25

3.4.2 The coupling apparatus is shown here.

3.4.3 The input wavelength can simply be altered by using a digital control.

3.5.1 Tilted Fiber Bragg Grating fabrication setup. $\quad$ _..............27

3.5.2 This setup can be used to produce gratings with tilts up to $30^{\circ}$.

3.5.3 UVS-652 Specialty Optical Fiber Product. Manufactured by CorActive ${ }^{\circledR} . \quad$................29 


\section{List of Figures (Continued)}

3.6.1 Fundamental and first-order harmonic modes, for $632 \mathrm{~nm}$ light.

3.6.2 A radiation profile showing spectral width of one diffracted mode for $488.0 \mathrm{~nm}$.

4.0.1 Intense blue light outcoupling observed for $30^{\circ} \mathrm{TFBG}$.

4.1.1 Efficiency and azimuthal angles of scattered modes for sample $10^{\circ}$ grating.

4.1.2 Sample green $(514.5 \mathrm{~nm})$ and blue $(488.0 \mathrm{~nm})$ radiation patterns are shown.

4.2.1 The agreement is clear between predicted and observed angular distribution.

4.2.2 Exponential relationship between outcoupled power and grating angle.

4.3.1 Corrections to and observations of fabrication process.

4.3.2 Strong visible outcoupling of UVS-652 $1 \mathrm{~cm} 30^{\circ} 1610 \mathrm{~nm}$ TFBG.

4.4.1 Polarization rotator was incrementally adjusted to test outcoupled radiation.

4.4.2 The angles of outcoupled radiation are shown and collected into similar modes.

5.1.1 The setup holding the phase mask was rotated to fabricate $30^{\circ}$ gratings. 


\section{List of Notations and Abbreviations}

\begin{tabular}{ll} 
CMT & Coupled Mode Theory \\
DM & Degenerated Mode \\
DMG & Degenerated Mode Group \\
EMI & Electromagnetic Interference \\
FBG & Fiber Bragg Grating \\
IR & Infrared \\
OSA & Optical Spectrum Analyzer \\
RI & Refractive Index \\
SRI & Surrounding Refractive Index \\
TFBG & Tilted Fiber Bragg Grating \\
TIR & Total Internal Reflection \\
UV & Ultraviolet \\
VCM & Volume Current Method \\
$\boldsymbol{\lambda}_{\mathbf{B}}$ & Bragg Wavelength \\
$\boldsymbol{n}_{\text {eff }}$ & Effective Refractive Index \\
$\boldsymbol{\Lambda}$ & Refractive Index Modulation Periodicity \\
$\boldsymbol{\theta}$ & Tilt Angle \\
\hline
\end{tabular}




\section{Chapter 1}

\section{Introduction}

\subsection{Fiber Bragg Gratings as Sensors}

It is not surprising that the need for sensors is a natural one. We all depend on our senses to perceive and interconnect with our surroundings and sensors are technological extensions of our natural senses. But they also allow us to "datify" the physical world and interact with it in new ways. With the development of new areas of technology comes the continuous demand to monitor, or sense, physical quantities such as temperature, strain, pressure, load, and a multitude of other parameters in order to maintain and operate our gadgets, machines and appliances. That is why, with the growing importance that sensors have in the world we live in, research and development in sensing technology is a high priority for the scientific community.

In sensing research, Fibre Bragg Gratings (FBGs) present important characteristics that set them apart including high sensitivity and multiplexing capacity as well as low fabrication cost. Fiber Optic sensors in general provide advantages over electrical sensors in that they are electrically passive and immune to electromagnetic interference (EMI).

In FBG, the periodicity of the grating is controlled using Ultraviolet (UV) inscription technology. This periodic modulation of the refractive index (RI) determines the wavelength of the light meant to be reflected back; the Bragg wavelength, $\lambda_{B}$. Reflectivity of $100 \%$ is possible as a result of phase matching between the in-coupled light and grating periodicity, and the shifts in this reflected Bragg wavelength can be used to measure an array of parameters 
including temperature and strain. The most appealing trait of these measurements is that FBG have a built-in referencing mechanism because the position of the Bragg wavelength is independent of the attenuation of signals in the system, therefore FBG sensors provide absolute quantities for the measured parameters. Notwithstanding the significant growth of FBG sensors in the last 35 years a new world of possibilities emerges when introducing a tilt to the grating planes of the FBG. This type of FBG is called a Tilted Fiber Bragg Grating (TFBG) and has very promising sensing capabilities.

For instance, in their planar form FBG cannot be used to measure Surrounding-material Refractive Index (SRI) since the reflected light is confined to the core mode. However, because the introduction of the tilt allows for backward-coupled cladding modes, these higher-order modes show up in transmission spectra as transmission loss peaks below the Bragg wavelength. In short, the TFBG is a device that senses its external environment while enjoying the same advantages as the untilted FBG.

\subsection{Tilted Fiber Bragg Gratings as Diffraction Prisms}

If a broadband visible light source is connected to a TFBG a phenomenon called diffraction would separate the incoming beam into different colours in a process that may be compared to the effect a prism on white light, although theoretically much different. Furthermore, when the tilt of the grating is large enough (above $30^{\circ}$ with respect to the fiber axis) there is significant light coupled into the higher-order diffraction modes, so there would be several orders of each colour coming out of the side of the fiber, such as in Figure 1.2.1 (see Appendix E for individual colour diagrams). 

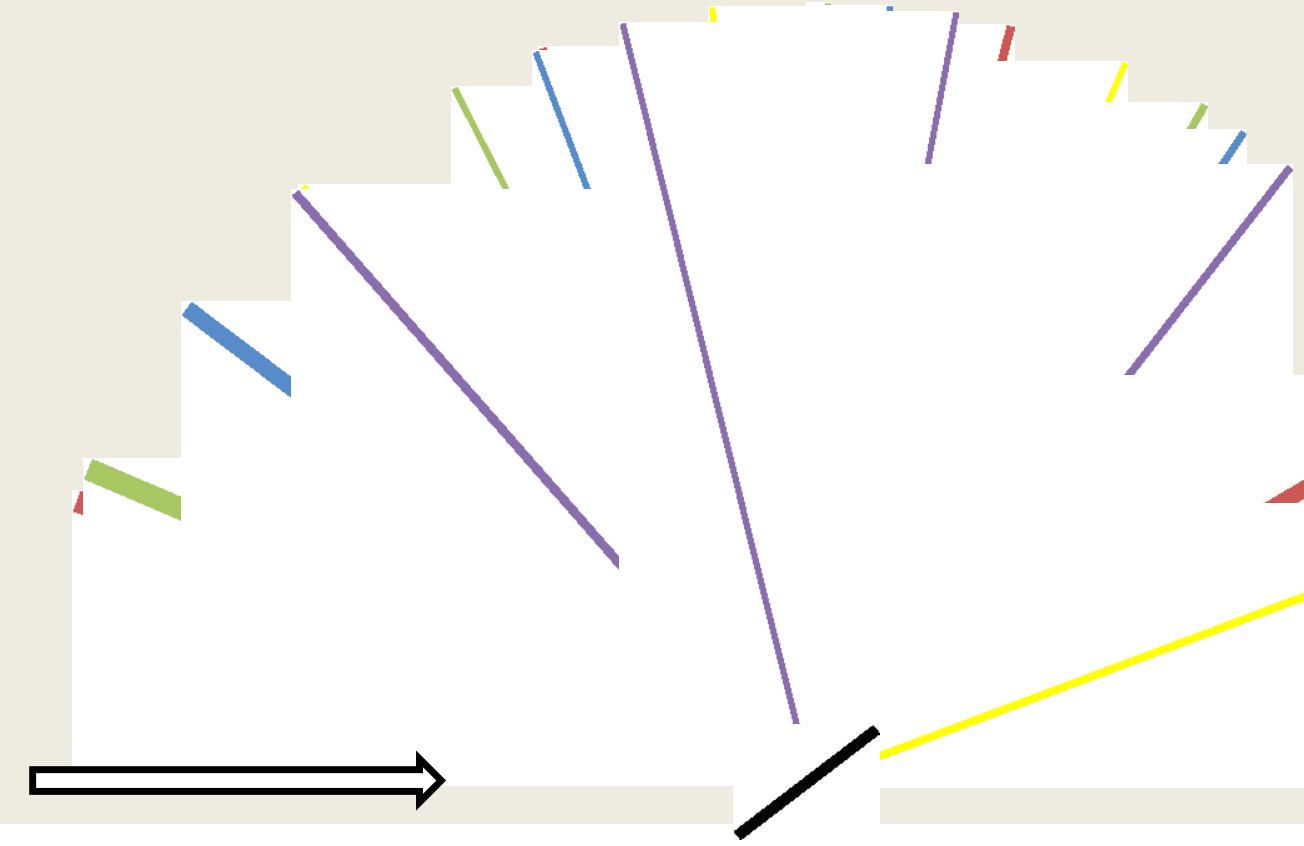

Figure 1.2.1 - A white-light source hitting a theoretical 1550nm FBG tilted by $37.5^{\circ}$.

In the scope of this thesis we investigate the sensitivity of such a TFBG sensor operating in the visible electromagnetic spectrum by studying the phenomenon of visible diffraction in TFBGs. The goal is to map output back to its input, namely the power and angular distribution of high-order diffraction modes (outputs) in relation to design characteristics such as the type of fiber used, the fabrication technique used to inscribe the TFBG into the fibre, and the pitch and tilt of the grating itself (inputs). The aim of such a study is to gain enough insight as to be able to reliably produce TFBGs with optimal visible light outcoupling. Though these results can be generalized to understand the behaviour of any colour in the visible spectrum, blue light will be emphasized for its particular relevance to ongoing research in molecular sensing ${ }^{1,2}$ as well as its reported enhancement of $\mathrm{Ag}$ nanocoated sensors ${ }^{3,4}$. As far as its applications, the variation in the outcoupling behaviour due to external changes in SIR could allow for novel visible light sensors never before created. This research serves as the underpinning for such an endeavour. 


\subsection{Organization of the Report}

In this project I investigate the interaction between the visible laser spectrum and a TFBG. I isolate for the effects of grating pitch, grating tilt, and cut-off wavelength on the azimuthal and longitudinal radiation profiles of outcoupled light. This comprehensive map is used to provide a broad understanding of the TFBG in the visible spectrum and potentially to help improve TFBG sensitivity in the visible range.

This report conveys the results obtained during the course of the Master's thesis at the Department of Electronics, Carleton University, Canada.

Chapter 2 contains background principles relating to FBG technology and serves as a summary of the fundamental methods and concepts behind TFBGs. The phase-matching criteria for sidetapping phenomena are reviewed along with the main methods used to understand grating diffraction. This chapter will serve as the elementary backdrop for understanding the latter results in the report, as well as the necessary explanation of the parameters to be tested.

In Chapter 3, I present an overview of the experiment, complete with a list of all the sources and components used in the apparatus, many of which are shown in diagrams. Reading this chapter is useful for anyone repeating the experiments described in this thesis.

Chapter 4 presents the azimuthal and longitudinal power distribution profiles versus wavelength for our TFBG. Results show that phase-matching conditions predict well the angular distribution, but not the power distribution of radiation modes for wavelengths from $457.9 \mathrm{~nm}$ to $647.1 \mathrm{~nm}$. Results confirm that a change in the grating pitch is strongly correlated 
to changes in the angular distribution of the modes as well as the out-coupled power. Since blue light is of particular importance, I also explore the effect of grating tilt on radiation distribution of blue light. I show that although the grating tilt does not significantly affect the angular distribution profiles of the radiated modes, it does have a strong impact on the power coupled into a specific mode.

In the course of this chapter, I use gratings inscribed in cladding suppressed fiber produced out-of-lab as references with which to compare several kinds of large-angle TFBGs made in our own laboratory. This chapter's main purpose is to summarize in graph and tabulated form all empirical results undertaken during the course of this project and to present the clear links between input and output.

Chapter 5 presents analysis and a discussion of the results from Chapter 4. To investigate the potential benefits of optimization, the parametric behaviour of several TFBG devices are considered. The conclusions drawn are used to predict a grating design range that may exhibit improved outcoupling in the blue light range. The main purpose of this chapter is to highlight the kind of TFBG fabrication setup needed to optimize the application of blue-light sensors.

Chapter 6 summarizes the thesis work and presents an outline for future work as well as considerations on innovative and versatile applications of our method in the engineering world; with a particular focus on blue light sensing in biochemical and DNA-detection technologies. 


\section{Chapter 2}

\section{Tilted Fibre Gratings in the Visible Spectrum}

Herein the Tilted Fiber Bragg Grating (TFBG) will be introduced as a special form of the more conventional Fiber Bragg Grating (FBG). First, FBGs will be presented as the overarching family of short period modulated gratings and their unique properties will be examined through coupled mode theory. In the latter part of the chapter I will focus on the TFBG in particular. The properties that the tilt angle $\theta$ exhibits will be discussed including cladding-mode coupling and how this leads to further interesting characteristics such as coupling to cladding modes and for large tilts, high-order diffraction. To put the results of this thesis project into academic perspective, this chapter details the different methods used to quantify grating diffraction as well as the methods underlying much of TFBG sensing research in the visible spectrum. The fabrication process of TFBG is highlighted in order to introduce the key concepts of grating pitch and grating tilt. It is shown that a simple phase matching approach is theoretically sufficient to explain some of the empirically derived results of this research.

\subsection{Fabrication}

In 1978 photosensitivity was discovered by Hill et $a l^{5}$ at CRC Canada. The team 'accidentally' came across this photosensitivity in optical fibers when laser light at $488 \mathrm{~nm}$ and $514.5 \mathrm{~nm}$ was coupled into a Ge-doped silica fiber. The phenomenon causes permanent changes to the refractive index in the fiber, and can thus be used to photo-induce gratings into optical fiber. 
This type of inscription results in a standing wave pattern in the fiber is known as a selforganizing grating. The disadvantage with this basic method is that the application of such gratings is limited since the reflected wavelengths are determined by the wavelength of the radiation used to create the pattern in the first place.

Later developments in photo-induction include a transverse holographic technique, known as dual-beam holography ${ }^{6,7}$. By intersecting two beams of UV light one can control the period of the inscribed gratings. The advantage of this technique is that a wide range of grating periods can be obtained. This development in grating inscription technology facilitated their emergence and popularization into applications and markets including aerospace, medical and general sensing technology domains.

Modern commercial production of FBGs uses phase-mask techniques to inscribe gratings in optical fiber. A phase mask with the desired periodicity simply covers the optical fiber and UV light is applied to the fiber to write the grating (no need for a laser source with high spatial coherence). Phase mask techniques not only allow for mass production of identical gratings by applying an individual mask, but also allow for variability in the grating period itself; making chirped FBG fabrication possible. In this technique the quality of the inscribed gratings is fully dependent on the quality of the mask, so imperfections in the mask are reflected in the grating.

In this thesis project I compare the outcoupling behaviour for gratings made with different types of fiber. The difference in fabrication technique may have a very important impact on the visible radiation patterns. In this research, however I focus on the impact of the types of fiber used for inscription, which is explored in more detail in Chapters $\mathbf{4}$ and $\mathbf{5 .}$ 


\subsection{Total Internal Reflection}

In order appreciate the more complex theoretical basis of TFBG properties, it is important to grasp the fundamental physical phenomenon upon which Fiber Optic cables work in general. Total Internal Reflection (TIR) serves as the backdrop for most inherent properties of FBG and TFBG alike.

When an EM wave crosses a boundary whereupon the refractive index changes, the light partially refracts and partially reflects as illustrated in Figure 2.2.1a. When crossing the interface from higher RI to lower RI, light refracts away from the boundary. For refractive indices $n_{1}>n_{2}$, it can be seen that as the incoming angle, $\theta_{i}$, increases the system will reach a point where the transmitted light travels along the boundary at a transmission angle, $\theta_{t}$, of $90^{\circ}$. For any incident light at an angle greater than this particular angle (called the critical angle, $\theta_{c}$ ) the light is no longer transmitted at all and it is totally reflected, as shown in Figure 2.2.1b.

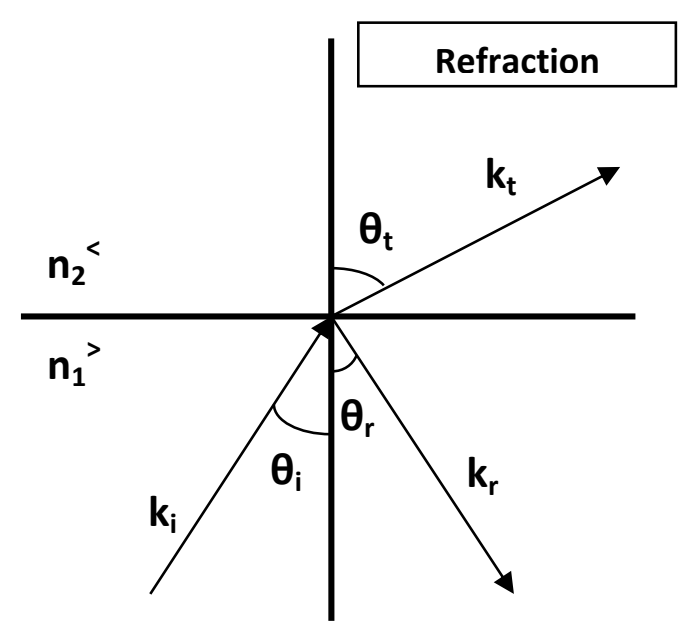

(a)

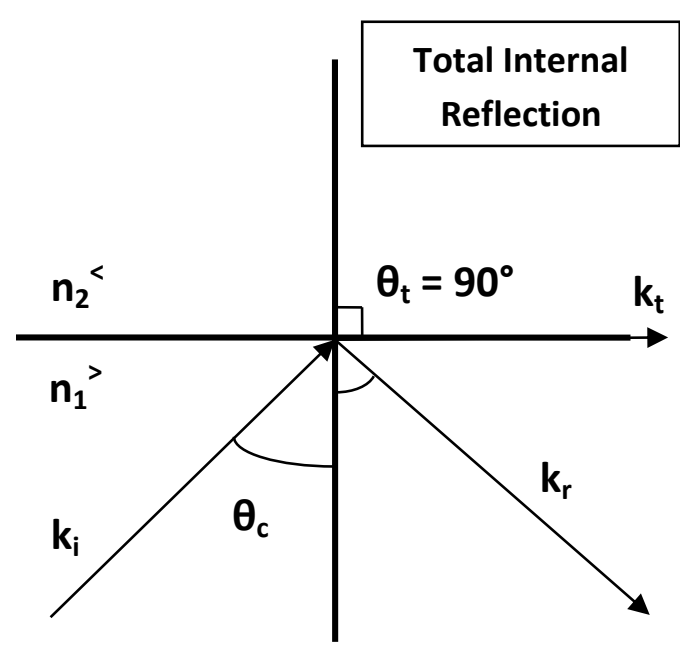

(b)

Figure 2.2.1 - Vector diagram highlights the difference between refraction and TIR. 
This phenomenon is known as Total Internal Reflection. Refraction at such a boundary is governed by Snell's Law:

$$
n_{i} \sin \left(\theta_{i}\right)=n_{t} \sin \left(\theta_{t}\right)
$$

When the incident beam reaches the critical angle the transmitted beam travels along the boundary perpendicular to the interface normal, as mentioned above. Substituting $90^{\circ}$ for the transmitted angle, I obtain a formula for the critical angle in terms of the internal and external refractive indices. At incident angles above this angle, TIR occurs.

$$
\theta_{c}=\sin ^{-1}\left(\frac{n_{t}}{n_{i}}\right)
$$

I will thus call upon the consequences of this phenomenon as they relate to TFBG sensing properties in subsequent chapters of the report.

\subsection{Simple Fiber Grating}

FBG is a periodic modulation of the refractive index in the core of an optical fiber where the planes of the inscribed gratings are perpendicular to the longitudinal axis of the fiber. The periodicity of this modulation is one half of the wavelength meant to be reflected; known as the Bragg wavelength.

$$
\lambda_{B}=2 n_{e f f} \Lambda
$$

where $\lambda_{B}$ is the Bragg wavelength, $n_{\text {eff }}$ is the effective refractive index of the grating in the core and $\Lambda$ is the grating period. This condition is known as the Bragg condition for the simplest case of a grating in single mode fiber. For this condition to be satisfied both energy and momentum 
must be conserved. For energy, the frequency of the incoming light must match that of the reflected light.

$$
h v_{i}=h v_{r}
$$

where $h$ is Planck's constant and $v$ represents frequency in units of $\mathrm{Hz}$. For momentum conservation the wavevectors involved must respect the following condition (see Figure 2.3.1):

$$
\boldsymbol{k}_{\boldsymbol{i}}+\boldsymbol{K}=\boldsymbol{k}_{\boldsymbol{r}}
$$

where $\boldsymbol{k}_{\boldsymbol{i}}$ and $\boldsymbol{k}_{\boldsymbol{r}}$ are the incident and reflected wavevectors respectively and $\boldsymbol{K}$ is the grating wavevector. The grating wavevector is perpendicular to the grating planes and has magnitude:

$$
K=2 \pi / \Lambda
$$

For pure reflection the reflected wave is exactly equal and opposite the incident wave. Since we know the wavevector $k_{i}$ will have magnitude $2 \pi n_{\text {eff }} / \lambda_{0}$, we rearrange (2.3.3) and substitute for magnitudes to find the resulting first order condition.

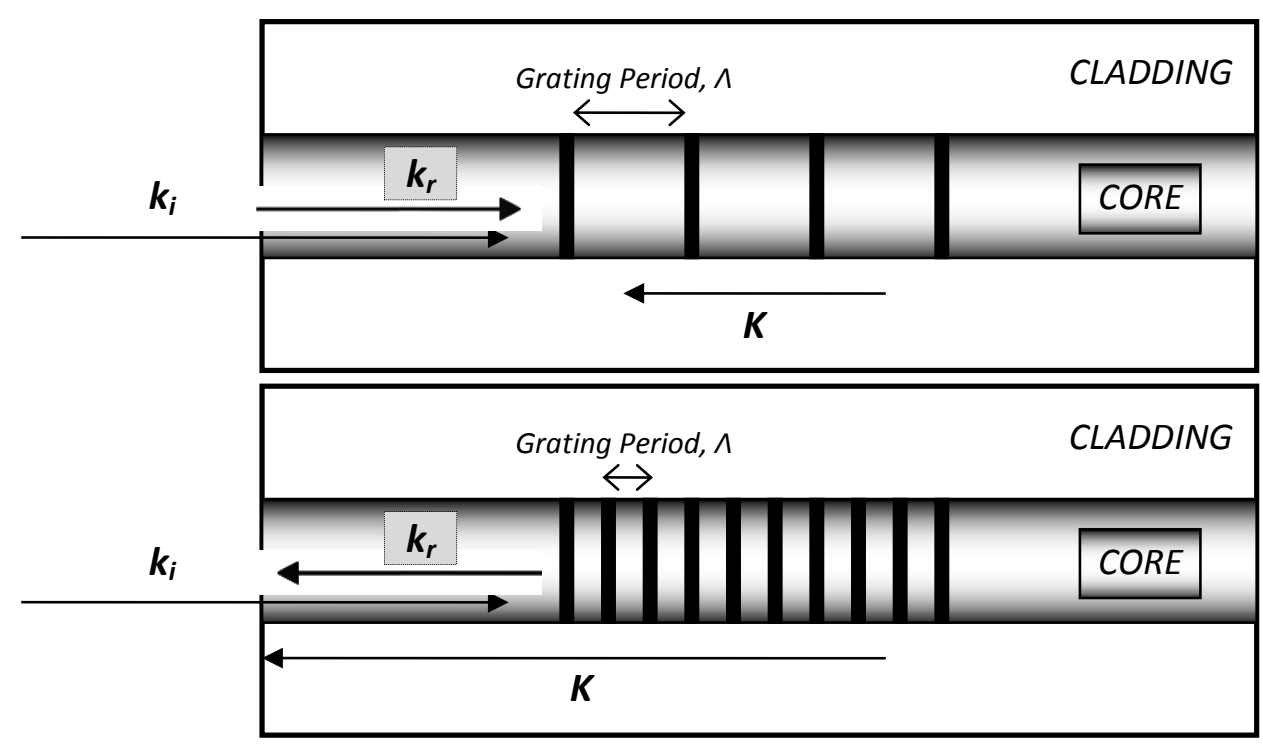

Figure 2.3.1 - Long (top) and short (bottom) period gratings. Incident, radiated and grating wavevectors shown. 


$$
\left(2 \pi n_{e f f} / \lambda_{0}\right)-\left(-2 \pi n_{e f f} / \lambda_{0}\right)=-(-2 \pi / \Lambda)
$$

The free-space wavelength that satisfies this phase matching condition is labeled the Bragg wavelength and from (2.3.5) the first-order Bragg condition in (2.3.1) is easily deduced.

\subsection{Fiber Bragg Grating Reflectivity}

Typically, the refractive index profile of a uniform grating in the core of a single mode fiber can be presented by the following equation ${ }^{5}$ :

$$
n(z)=n_{a}+\Delta n \cos \left(\frac{2 \pi z}{\Lambda}\right)
$$

where $n_{a}$ is the average refractive index of the core, $\Delta n$ is refractive index perturbation amplitude (typically in the range of $10^{-4}$ to $10^{-3}$ ), and $z$ is the distance along the fiber longitudinal axis. To find reflectivity, one must begin with the Helmholtz equation:

$$
\nabla^{2} \tilde{E}+\tilde{n} k_{0}^{2} \tilde{E}=0
$$

where $\tilde{E}$ is the Fourier Transform of the electric field distribution with respect to time.

Since $n(z)$ is periodic in the $z$ direction, reflectivity can be found by considering the Fourier Series expansion:

$$
n(z)=\sum_{m=-\infty}^{+\infty} n_{m} \exp [2 \pi i m(z / \Lambda)]
$$

By applying appropriate boundary conditions, reflectivity can be expressed as following (Figure

2.4.1) - see Appendix A for more detail: 


$$
R(l, \lambda)=\frac{\kappa^{2} \sinh ^{2}(s l)}{\delta k^{2} \sinh ^{2}(s l)+s^{2} \cosh ^{2}(s l)}
$$

where $I$ is the grating length, $k$ is the coupling efficiency, $\delta k=k-\pi / \lambda$ represents wavevector detuning (while the propagation constant $k=2 \pi n_{a} / \lambda$ ) and $s^{2}=k^{2}-\delta k^{2}$. For the center wavelength, there is no detuning; $\delta k=0$, so the maximum reflectivity is given by:

$$
R_{\max }(l, \lambda)=\tanh ^{2}(\kappa l)
$$

In practice, reflectivities of over $99 \%$ at the center wavelength are achievable and commonplace. The undesirable feature of FBG reflection spectrum is the presence of the sidelobes on either side of the stop band.

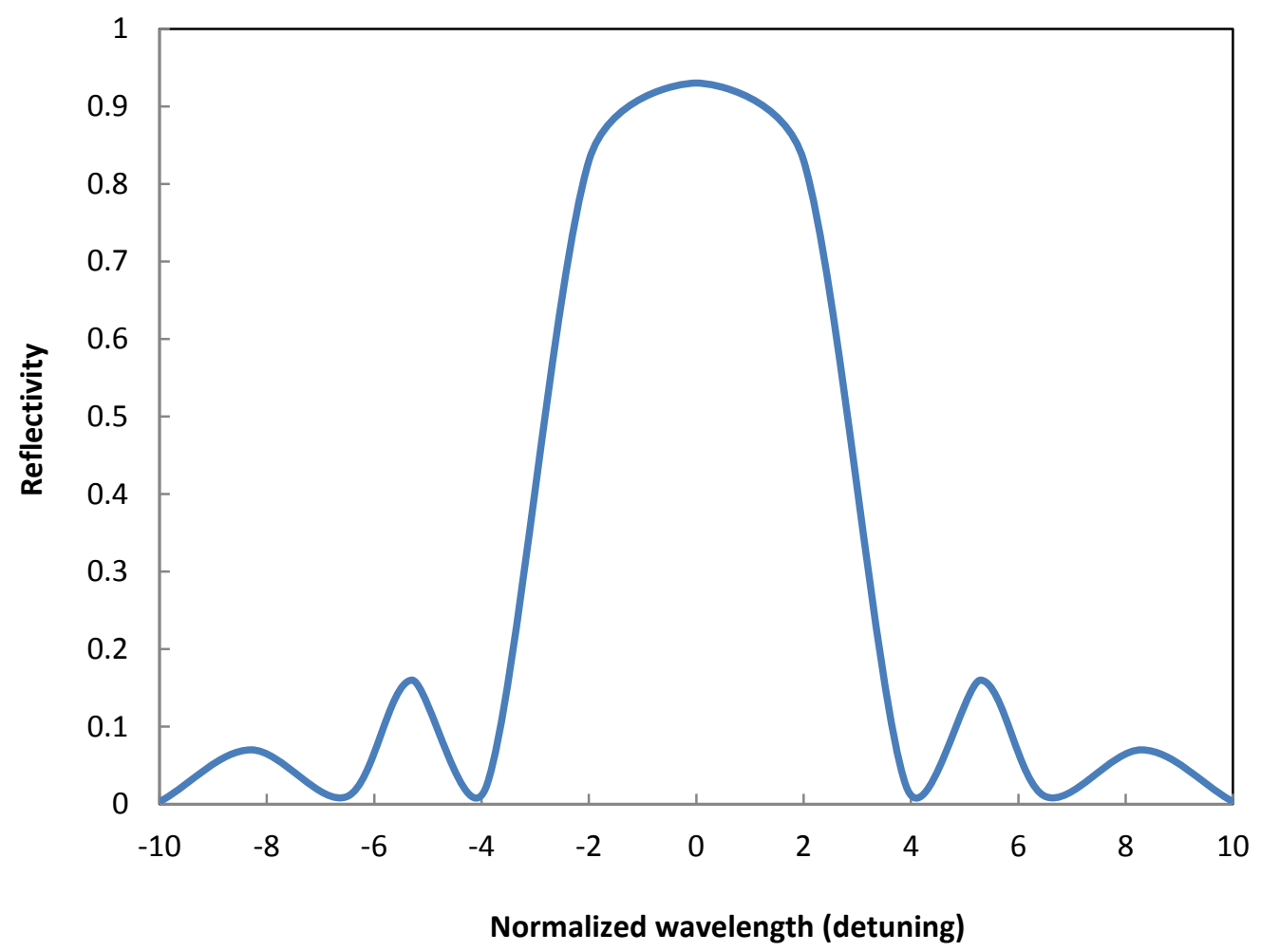

Figure 2.4.1: Sample reflection spectrum for FBG versus detuning from center wavelength. 


\subsection{Tilted Fiber Bragg Grating}

TFBGs are simply a special type of short-period FBG at visible wavelengths in which the grating planes are slanted with respect to the longitudinal fiber axis. Figure 2.5.1 shows a tilted grating with an effective grating pitch $(\Lambda)$ that remains the same along the fibre axis as compared to a flat grating.

\section{Flat Grating}

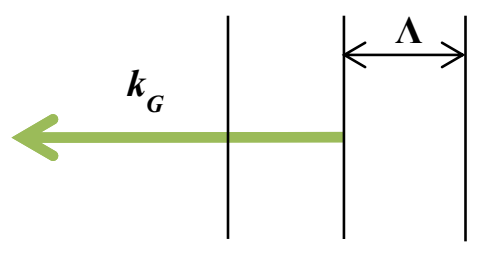

Tilted Grating

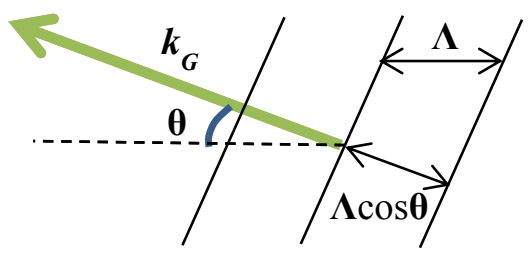

Figure 2.5.1 - The effective grating periodicity remains unchanged along the fiber axis.

As mentioned in Chapter 1, the tilt of the grating planes allows them to couple light from the forward propagating core mode to backward propagating core and cladding modes. This results in transmission loss peaks at discrete resonant wavelengths shorter than the Bragg wavelength as shown in Figure 2.5.2. The cladding modes travel near the cladding-external medium interface which renders them sensitive to SRI.

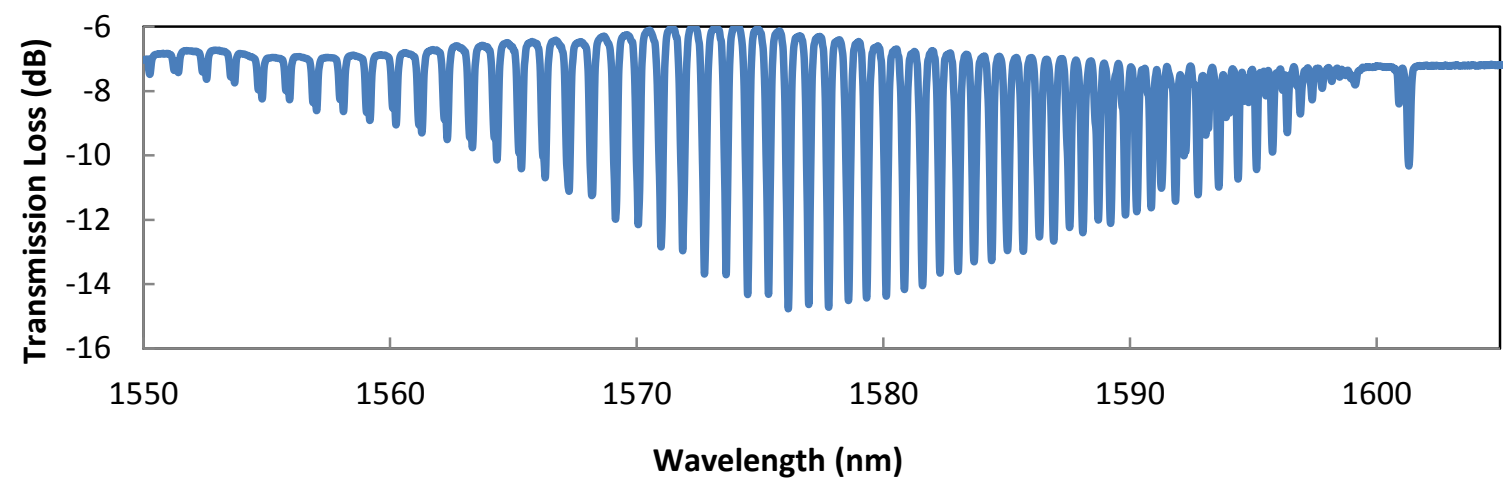

Figure 2.5.2 - A typical TFBG transmission spectrum showing higher order mode losses. 
When the grating plane is tilted with respect to the fiber axis as shown in Figure 2.5.3, the phase matching condition predicts tilt and wavelength dependent diffraction. The three wavevectors of interest are the incoming, outgoing and grating wavevectors, such that mode coupling between incident and radiated waves is related to the grating wavevector.

By establishing a coordinate system to separate the axial and normal components we are able to explicitly derive the phase matching conditions. Along the z-axis, we add the zcomponents as follows:

$$
\begin{gathered}
k_{I}-k_{G} \cdot \hat{z}=k_{R} \cdot \hat{z} \\
\frac{2 \pi n_{e f f}}{\lambda}-\frac{m \pi}{\Lambda \cos \theta} \cdot \cos \theta=\frac{2 \pi n_{\text {core }}}{\lambda} \cdot \cos \alpha
\end{gathered}
$$

Tilt angle of the grating is eliminated from the equation because as the tilt increases so does the size of the grating wavevector, effectively canceling this term as shown in (2.5.3).

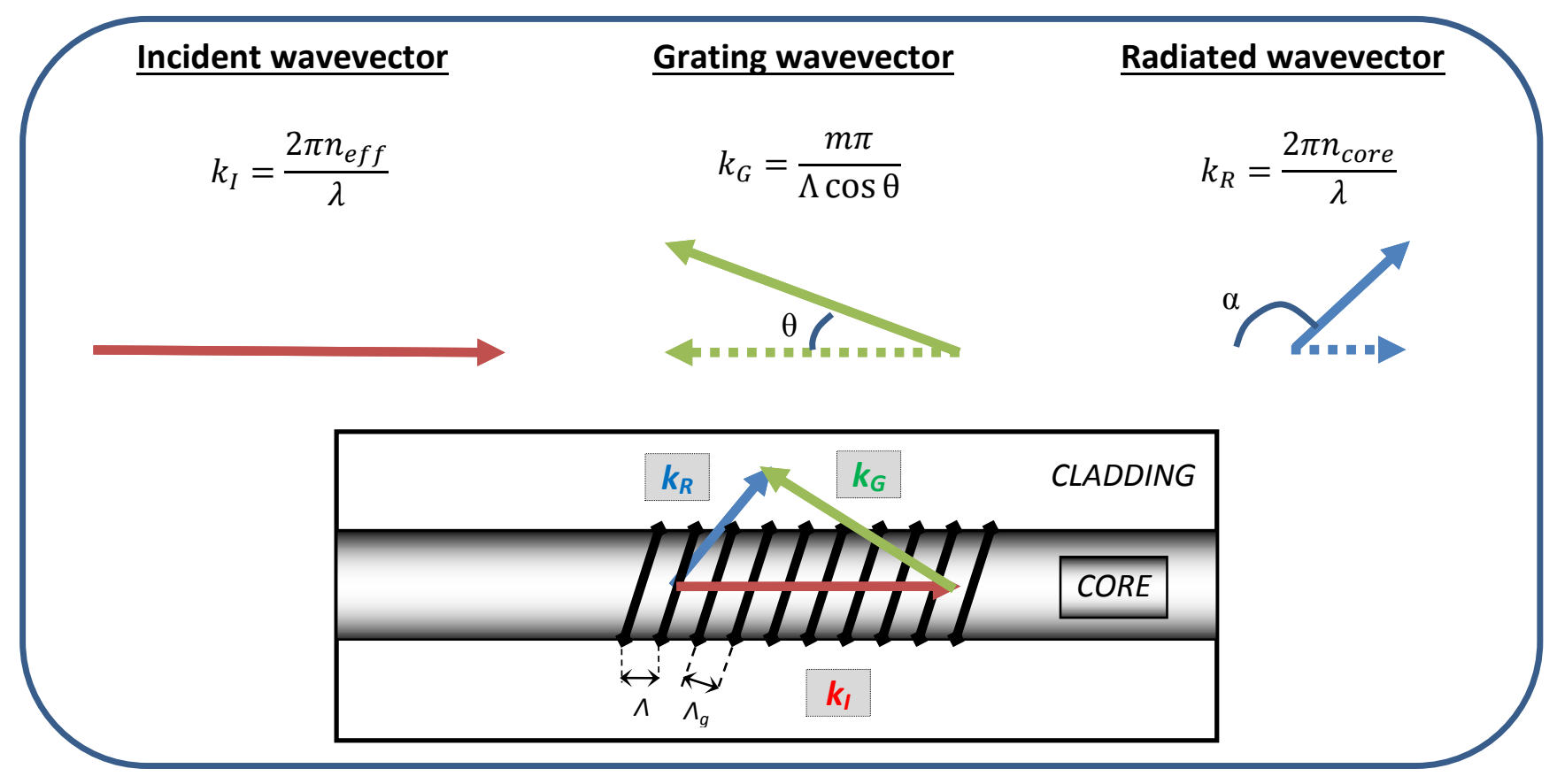

Figure 2.5.3 - Phase matching analysis of incoming $\boldsymbol{k}_{i}$, grating $\boldsymbol{k}_{G}$ and radiation $\boldsymbol{k}_{\boldsymbol{R}}$ wavevectors. This diagram applies only to short period gratings. Analyis for long period gratings differs. 
Again, we see that the tilt of the grating does not actually affect the z-component of the phase-matching condition. Isolating for the angle, $\alpha$, at which the diffracted wave exists the fiber as a function of incoming wavelength $(\lambda)$ and diffraction order $(m)$ we get:

$$
\alpha_{m}(\lambda)=\cos ^{-1}\left(\frac{\Lambda 2 \pi n_{e f f}-m \lambda}{\Lambda 2 \pi n_{\text {core }}}\right)
$$

This equation predicts the angle of the outcoupled radiation as a function of wavelength, mode number, and effective and core refractive indices. In Chapter 4, these predicted angles are compared with experimentally measured angles. Snell's law was taken into consideration as the outcoupled radiation crosses the fiber-air interface. We note importantly that only the transverse component of the phase-matching condition is affected by the tilt angle, alpha. This implies the longitudinal phase-matching condition is 'unaffected' by the grating tilt. This is supported since the gratings that were made used a fabrication configuration that maintains a constant Bragg wavelength ${ }^{8}$. In contrast with this coupled-wave approach, there is also the Volume Current Method approach adopted or explored by certain groups. ${ }^{9-13}$ The former coupled-wave approach is also adopted by other groups to explain optical fiber sidetap phenomena. ${ }^{13-21}$ In Kashyap's model ${ }^{17}$ (Section 2.7) the net filter response, or power, of the diffraction grating depends on the degree of both longitudinal and transverse phase matching. Also, in this very same model the conditions are shown for Bragg reflection, back reflection to cladding modes, forward coupling to cladding modes, coupling to radiation modes and the effect of higher order $(\mathrm{m})$ grating wavector. The effect of higher-order m number is equivalent to a longer period grating which results in forward coupling, hence explaining the multiple diffraction orders. A rigorous derivation of these fundamental cases is given in Tamir $(1975)^{25}$. 


\subsection{Visible Diffraction Efficiencies in Tilted Fibre Bragg Gratings}

Many researchers have been involved with the progress to date of our understanding of visible light diffraction behaviour in fibre optics. Within the theoretical sphere, several useful methods called Volume Current Method ${ }^{9-13}$, Coupled Mode Analysis ${ }^{9,14-15}$, and a Free Space Model ${ }^{16-17}$ are used interchangeably since either one has pros and cons. Volume Current Method (VCM) is an analytic approach to calculate the radiation pattern of tilted fiber gratings. Since the Coupled Mode Theory (CMT) inherently breaks down for radiation perpendicular to the fiber axis, and since this particular configuration is of great interest for grating outcoupling, the VCM as an approach is often better suited as a design tool for this purpose. However, this research is experimental in nature and not confined to perpendicular radiation so the theoretical derivations of the VCM are considered outside the scope of this research, though information on the VCM is readily accessible in the literature ${ }^{9-13}$.

A subset of antenna theory is used in Kashyap's ${ }^{17}$ model for power efficiency of visible diffraction in TFBGs and discuss the difficulties in implementing this theoretical framework.

Figure 2.6.1 presents a schematic of the problem under study. The scattered light creates a cone defined by the phase matched angle $q$ (labeled $\phi$ in the remainder of this thesis). By considering a fibre with an infinite cladding $\left(\mathrm{n}_{\mathrm{co}}, \mathrm{n}_{\mathrm{cl}}\right)$ with a UV written FBG with a period $\Lambda$ tilted under the angle $\theta_{\mathrm{g}}$ along the $\mathrm{y}$-axis, the scattered power from the grating is given by integrating all radiating dipoles through the spherical surface with radius $\mathrm{R}$ :

$$
P_{\text {scat }}(\lambda)=\int_{\phi=0}^{2 \pi} \int_{q=0}^{\pi} R^{2} S_{\text {scat }}(R, \phi, q, \lambda) \sin q d q d \phi
$$




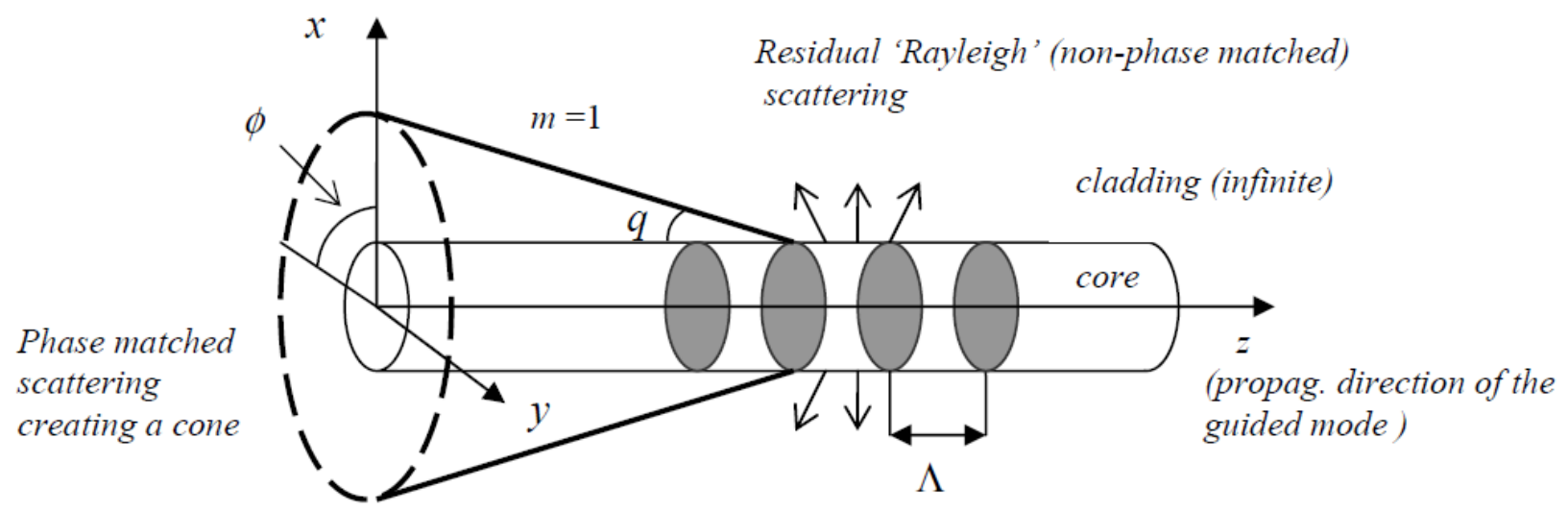

Figure 2.6.1 - Scattering from the fibre core with the FBG. Light scatters in cone shape. ${ }^{17}$

with $q$ being the angle between the fibre axis $(z)$ and the direction of the radiation, $\phi$ here being the azimuthal angle ( $x-y$ plane) and $\lambda$ being the wavelength of the interacting light. The Poynting vector is defined through:

$$
S_{\text {scat }}(R, \phi, q, \lambda)=\frac{n_{c l}}{2}\left(\frac{\varepsilon_{0}}{\mu_{0}}\right)^{1 / 2}\left|E_{\text {scat }}(R, \phi, q, \lambda)\right|^{2}
$$

The scattered field can be written as follows:

$$
E_{\text {scat }}(R, \phi, q, \lambda)=\frac{\Gamma}{R} \iint_{\text {core }} E_{0}(x, y) I_{L}(x, L) e^{-i k n_{c l}(x \cos \phi \sin q+y \sin \phi \sin q)} d x d y
$$

with $\Gamma=k^{2} n_{c l} \Delta n e^{i k n_{c l} R} / 2 \pi, k=2 \pi / \lambda$, and $\Delta n$ is the maximum refractive index change in the grating. Integration is over the fibre core area. Finally, the function depending on the total length of the grating, defined as

$$
I_{L}(x, L)=\frac{e^{i \gamma x}}{2} \frac{e^{i v L}-1}{i v}
$$

will lead to longitudinal phase matching (see previous derivation in Section 2.5) where $q_{L}$ is the angle at the longitudinal resonance. For the sake of simplicity, Kashyap considers $m=1$. 
Rewriting (2.6.3), the scattered field can be written as follows:

$$
E_{\text {scat }}(R, \phi, q, \lambda)=\frac{\Gamma}{2 R} \frac{e^{i v L}-1}{i v} I_{\text {core }}(\gamma, q, \phi)
$$

where the function $I_{\text {core }}$ is the non-trivial integration expression:

$$
I_{\text {core }}(\gamma, q, \phi)=\iint_{\text {core }} E_{0}(x, y) e^{\left\{i\left[x\left(\gamma-k n_{n c l} \cos \phi \sin q\right)-y k n_{c l} \sin \phi \sin q\right]\right\}} d S
$$

When (2.6.6) is converted into polar form and substituted into (2.6.5), the scattered power:

$$
P_{\text {scat }}(\lambda)=\frac{n_{c l}}{2}\left(\frac{\varepsilon_{0}}{\mu_{0}}\right)^{1 / 2} \Gamma^{*} \Gamma \int_{\phi=0}^{2 \pi} \int_{q=0}^{\pi}\left|I_{\text {core }}(\gamma, q, \phi)\right|^{2} \frac{\sin ^{2}(v L / 2)}{v^{2}} \sin q d q d \phi
$$

This model correctly predicts that the $m=1$ mode is dominant, and this final form of scattered power is very promising for future theoretical considerations, however the $I_{\text {core }}$ parameter is not trivial as it requires a complex analysis further explained in Kashyap's model. Furthermore, this derivation ignores the z-component of the electric field, which according to literature ${ }^{13}$ is not negligible for this phenomenon.

Finally, the experimental observations mentioned in this model did not clearly indicate whether these equations can be used to model all visible wavelengths as the reported validation is only at HeNe wavelength $(632 \mathrm{~nm})$.

For these reasons, this model is considered a good starting point for future work on theoretical comparisons of experimentally observed efficiency measurements reported in Chapter 4 of this thesis, though it was beyond the scope of this experimental thesis to use this model. 
From Kashyap ${ }^{17}$ the phase matching for FBG for non-zero tilt shows the phase matched angles contain the major part of the radiated power and are expressed as:

$$
q_{L}=\cos ^{-1}\left[\frac{1}{n_{c l}}\left(\frac{m \lambda}{\Lambda} \cos \theta_{g}-N\right)\right]
$$

This equation is analagous and identical to the simple phase-matching derived equation (2.5.3).

\subsection{Conclusion}

The Fiber Bragg Grating as a basic component in optical sensors was introduced. The tilting of the grating planes presents interesting properties in terms of sensing and flexibility. The coupling of the core mode into backward coupled cladding modes renders these latter modes 'leaky' to external media. This process is rendered more significant by large-tilt FBGs (greater than $30^{\circ}$ ) due to the high coupling efficiencies into these higher order modes. In summary, TFBGs present extraordinary possibilities due to their versatility with regards to sensing and their ability to control crosstalk between the confined core mode and the more externally interactive cladding modes. 


\section{Chapter 3}

\section{Test Apparatus for Visible Diffraction in TFBG}

This work began as part of the MOSAIC (Multimodal Optical Sensor Applications, Interfaces, and Controls) project, a federal-private-academic collaboration sponsored by NSERC. The focus area of this thesis in relation this wider project was the investigation of TFBG mode-coupling phenomena in the visible light range. Relative to common NIR bands used heavily in optical fibre communication, this particular band of light has not been deeply studied. Hence the need for an original and empirical probe into the effects of visible light radiation through, and outtapped from, a Tilted Fibre Bragg Grating. In this chapter I look at the original apparatus design, followed by an explanation of the fabrication techniques used to generate various kinds of test fibres. Finally, the data analysis techniques are presented to establish the validation framework to compare empirical findings with theory, and the apparatus is shown to be an effective and versatile measurement tool for TFBG Visible Light Radiation.

\subsection{Feasibility Study Using HeNe Laser}

Before the main apparatus was finally developed, the initial work went into testing for visible light outcoupling, as shown in Figure 3.1.1. A camera was used to observe the images shown in Figure 3.1.2., which proved that the setup design could work to observe diffraction maxima. The mounted camera was rotated by an angle $\theta$ around the $x$-axis on the $y$ - $z$ plane until maxima were observed. A rotator was used to rotate the fibre around the z-axis to account for the 
polar $(\psi)$ dependence of the radiation patterns, discussed in chapters 4 and 5 . This served as the necessary validation to test the concept because this meant I could now replace the camera with a power meter. By sweeping the camera in the place of the power meter, we were able to reduce all output parameters into one single multivariable output. The output power as a function of input power $\left(P_{\text {in }}\right)$, azimuthal angle $(\theta)$, polar angle $(\psi)$ and input wavelength $(\lambda)$, TFBG slant $(\phi)$ and TFBG pitch $(\Lambda)$ can be measured as long as any apparatus design is able to adequately control, isolate and vary for each of these 6 parameters. This output is described by the function $P_{\text {out }}\left(P_{\text {in }}, \theta, \psi, \lambda, \phi, \Lambda\right)$.

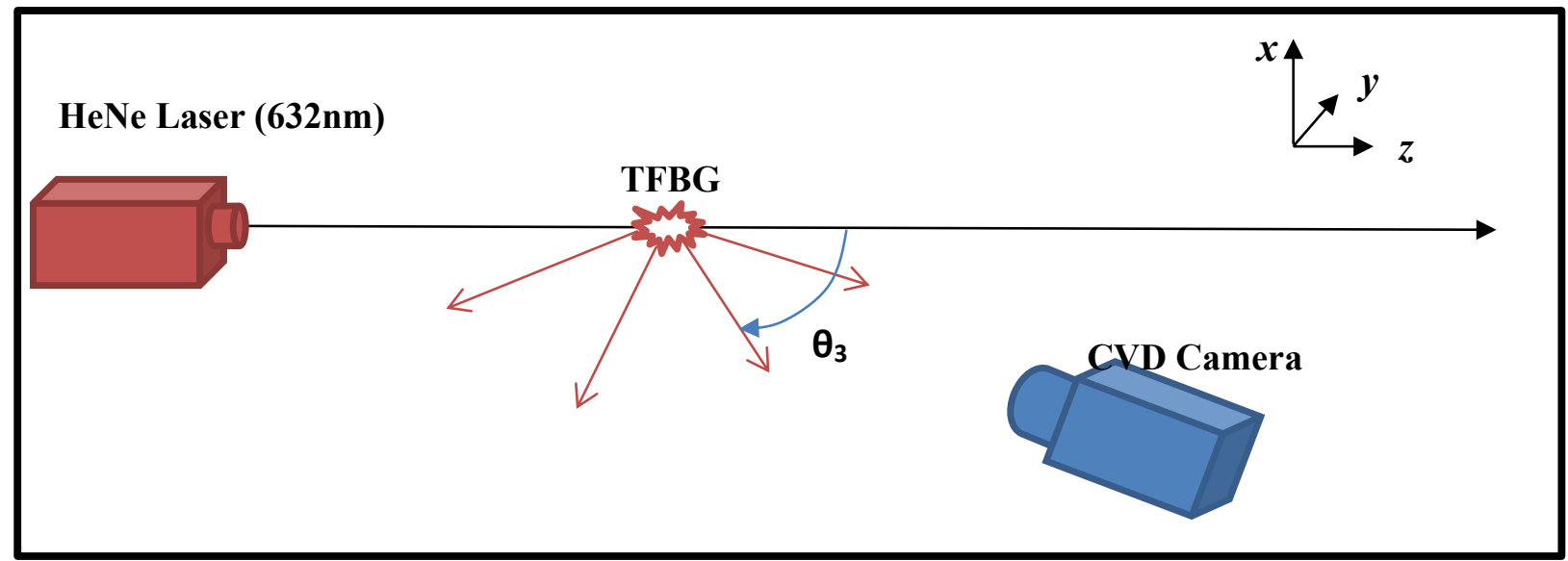

Figure 3.1.1 - The idea was to test the feasibility of visible light diffraction in standard SMF-28 fibre.

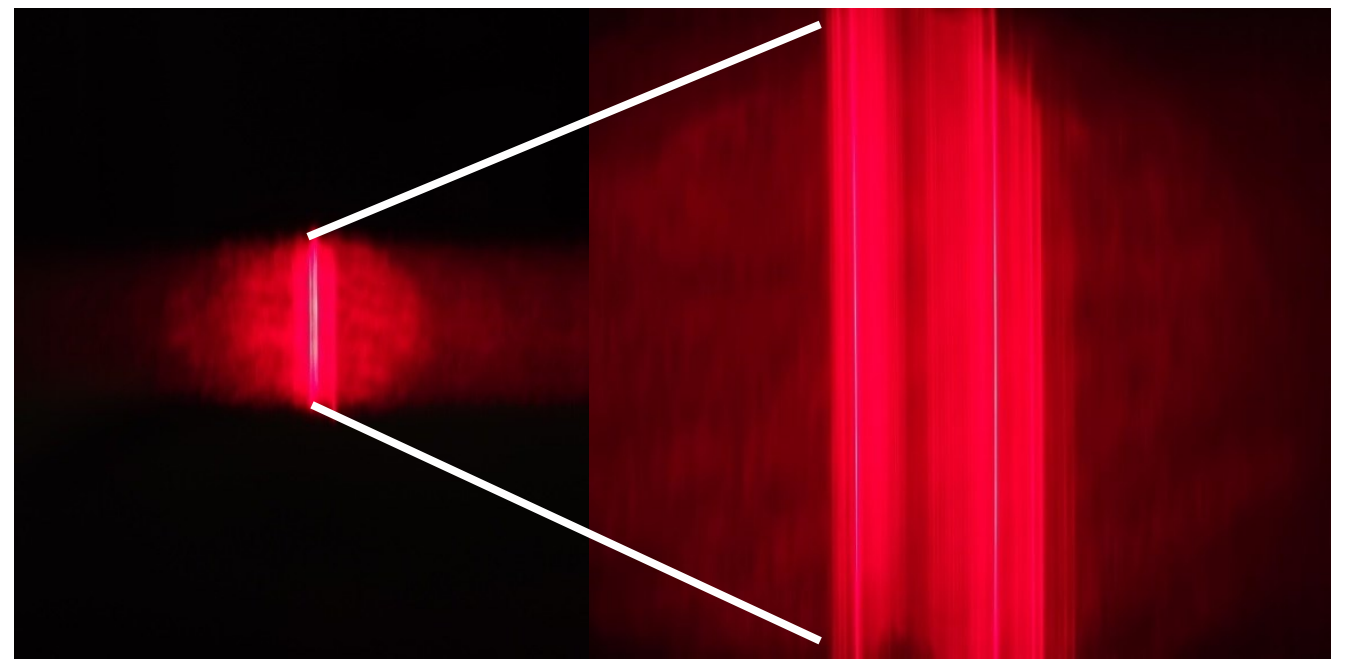

Figure 3.1.2 - A sample of a radiation maximum $(m=1)$ observed at a particular angle $\theta_{m}$. 


\subsection{List of Apparatus}

The list of apparatus used in the main experimental procedure is as follows (a schematic is shown below in Figure 3.2.1 indicating where the instruments are located relative to the fibre):

1. CVD Camera

- For initial verification of radiation peaks (Section 3.1)

2. Goniometer

- To measure diffracted angles

3. Circular Aperture Optical Power Meter - Attached to viewing end of Goniometer

- To measure power of diffracted modes

4. Fibre Holder with Rotator

- To hold fibre in place while allowing polar rotation

5. SMF-28 Fibre

- Standard single mode fiber

6. UVS-INT Fibre

- A type of cladding mode suppressed fibre

7. UVS-652 Fibre

- A type of cladding mode suppressed fibre

8. Coherent $^{\circledR}$ Innova $70 C$-Spectrum - Tunable laser (458 nm - 753 nm)

- To control input power and input wavelength

9. Polarizor

- To control input polarization

10. TFBG

- Produced with varying tilt angle and Bragg wavelength

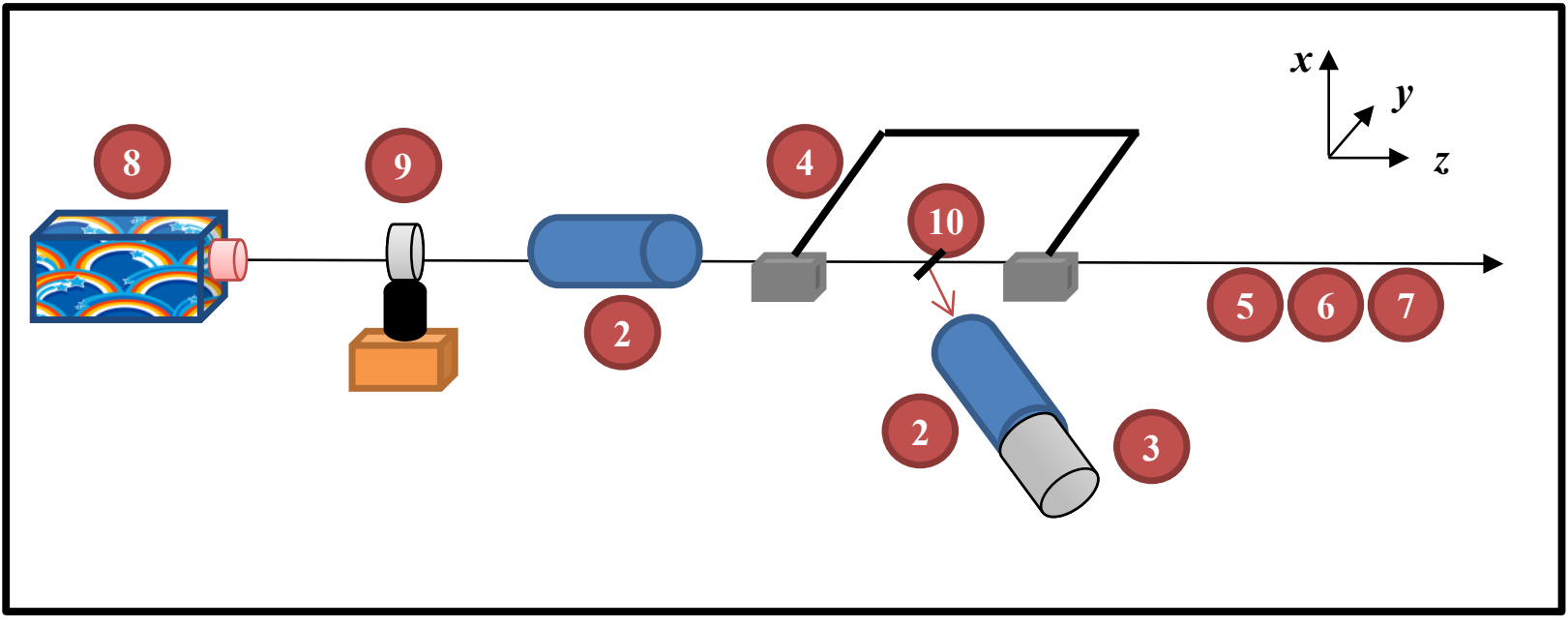

Figure 3.2.1 - Schematic of test apparatus with main test items identified. 


\subsection{Apparatus Design - Assembly and Procedure}

As explained in section 3.1, an original setup was required to empirically probe the parameters involved with diffraction of visible light radiation in a Tilted Fibre Bragg Grating. A Coherent Spectrum $70 \mathrm{C}$ laser was used to control both the power and the wavelength of the input light. A Goniometer and a fibre holder were assembled into the apparatus shown below. This $180^{\circ}$ Goniometer combined with a polar-rotating fibre stand allowed our setup the complete 3D freedom needed to measure the output radiated power in relation to all 6 input parameters; wavelength $(\lambda)$, power $\left(P_{\text {in }}\right)$, azimuthal angle $(\theta)$, polar angle $(\psi)$, grating tilt $(\phi)$ and pitch $(\Lambda)$.

In order to assemble the design shown in Figure 3.3.1, the TFBG was slotted through the fixed end of the Goniometer, at the centre of which a fibre holder was fixed in place used optical screws to ensure stable angle measurements. A small U-shaped metal rod was inserted into the fibre holder in order to rotate the fibre to measure polar, or azimuthal, dependence.

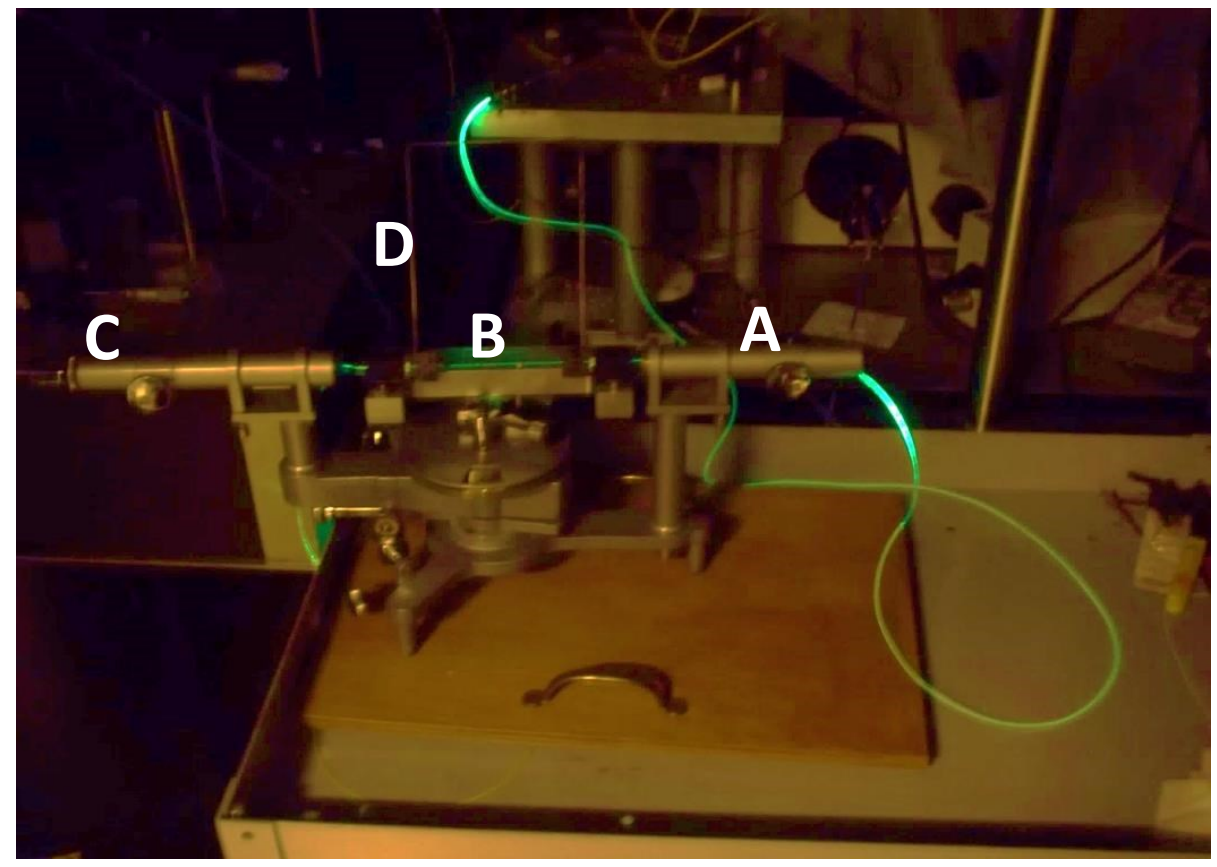

Figure 3.3.1 - TFBG is at the centre of the fibre holder (B). The left side of the Goniometer (C), replacing the CVD camera, was rotated around the TFBG. The rotator (D) was used to measure polar depedence. 


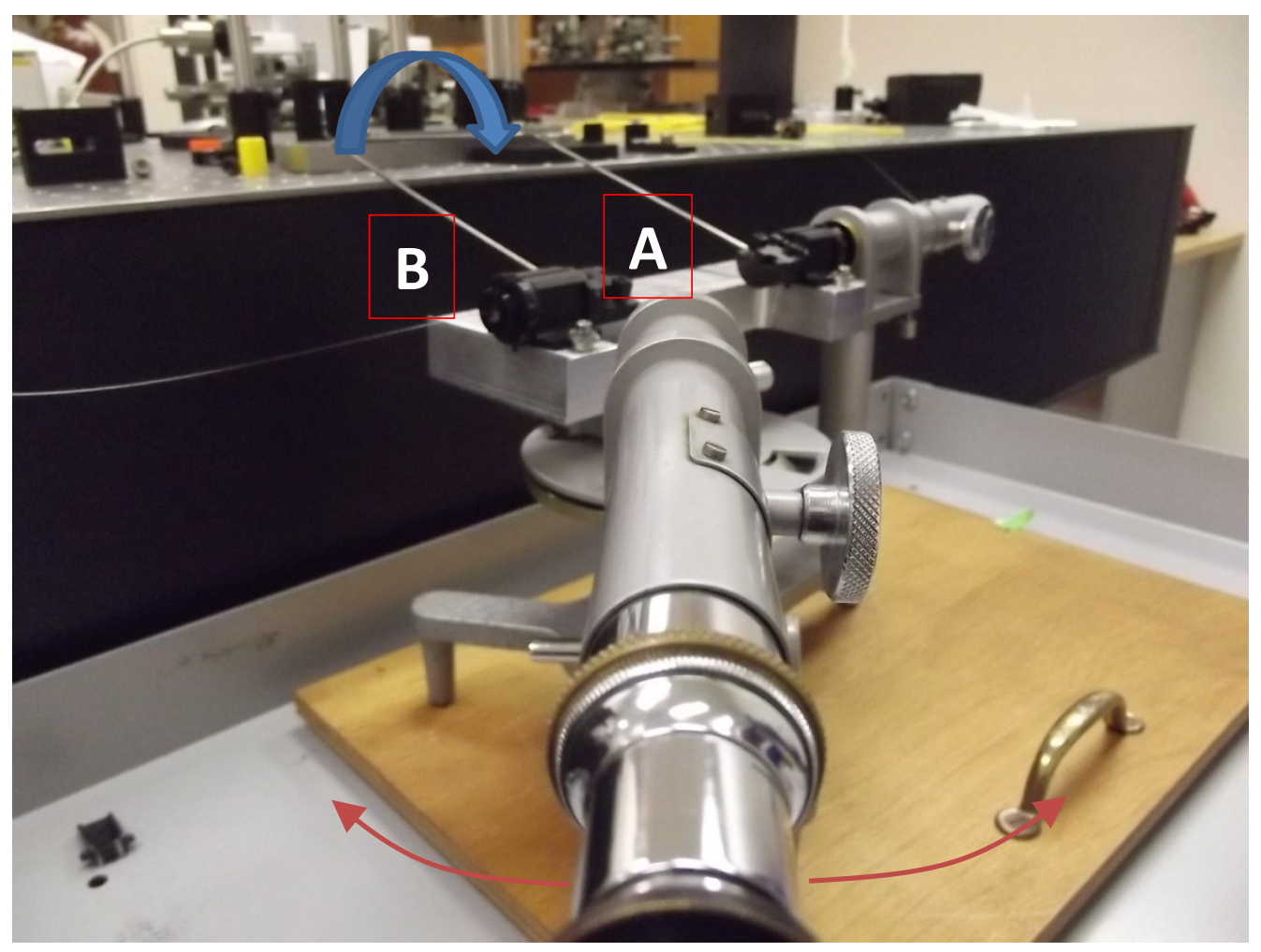

Figure 3.3.2 - This picture illustrates clearly how the observing end of the Goniometer was used to rotate (red arrows) around the TFBG (A) at the centre of the fibre holder. A power meter was used to replace the observing lens in order to 'scan' the entire $180^{\circ}$ range provided by the setup. The fibre holder was rotated using the rotator (B).

Once validated and tested, this apparatus proved to be the main data source for this thesis due to its versatility. Shown in Figure 3.3.2 are the two angular ranges that allow the TFBG to be seen "in 3D" by rotating the viewing end of the Goniometer as well as the fibre rotator shown as " $\mathrm{B}$ " in the above figure. It is important to stress that the fibre holder must be attached to the Goniometer rigidly in order to have reproducible test results. The novel data collected from these tests were analyzed and compared favourably to theoretical expectations. Due to time constraints, the relationship between input and output power losses could not be adequately tested within the length of this research. These tests are encouraged and any future work may benefit from the continuation of the original approach used in this project. 


\subsection{Controlling Beam Wavelength and Power}

Figure 3.4.1 shows the Coherent ${ }^{\circledR}$ Spectrum-70C Innova Laser used in this experiment. The freespace-to-fiber coupling setup is also shown in Figure 3.4.2. Finally, in Figure 3.4.3, the variation in input wavelength $\left(\lambda_{L}\right)$ is highlighted by showing the laser tuned to blue, green and red. For the purposes of this comparative research five colours were used (indigo $458 \mathrm{~nm}$, blue $488 \mathrm{~nm}$, green $515 \mathrm{~nm}$, yellow $568 \mathrm{~nm}$ and red $647 \mathrm{~nm}$ ). These were used to validate the observed diffraction behaviour versus that predicted by phase-matching conditions.

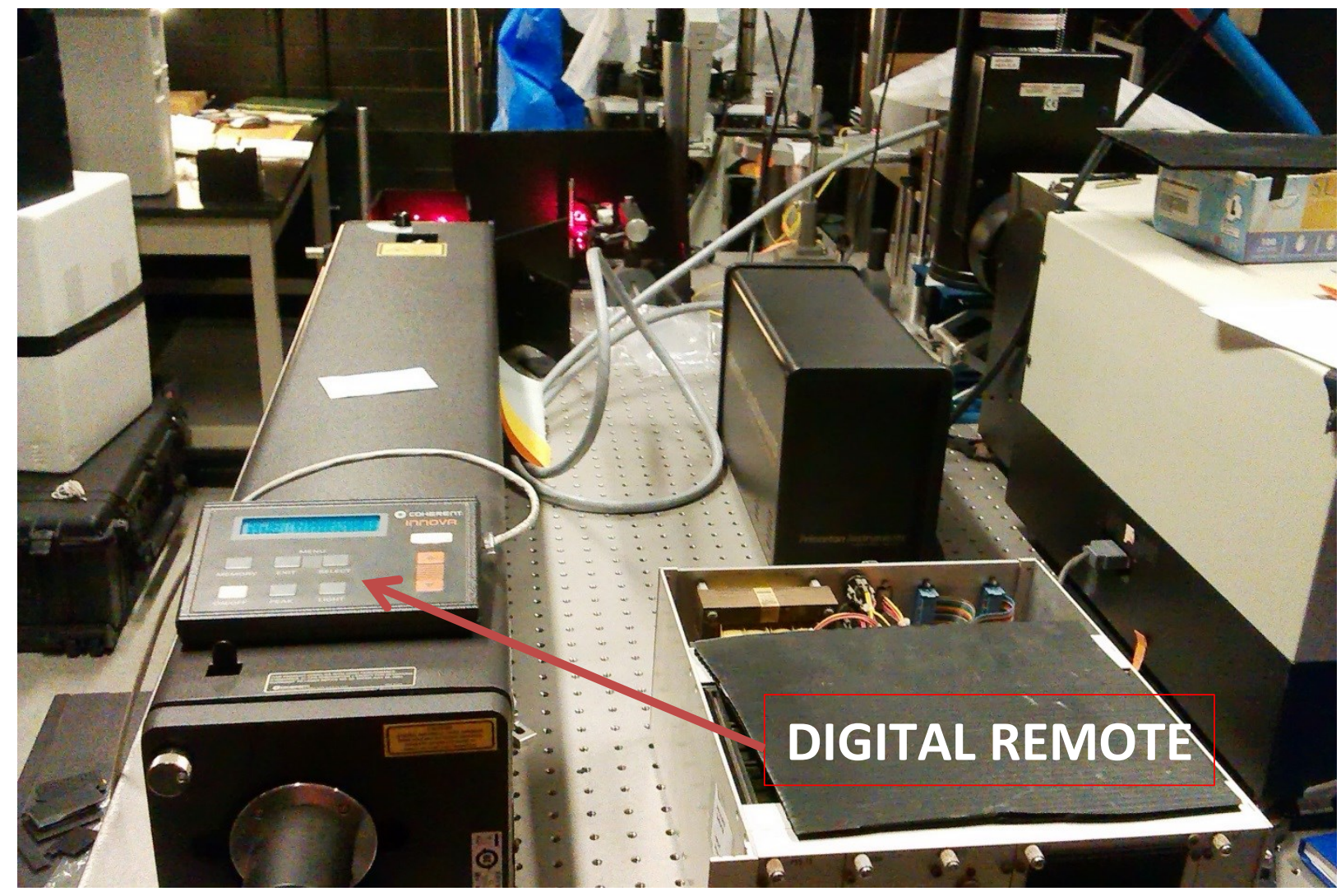

Figure 3.4.1 - The COHERENT SPECTRUM 70C laser, to the left, is adjusted using a digital interface above. Both power and wavelength can be finely tuned. The laser here is tuned to red - or $647 \mathrm{~nm}$. 


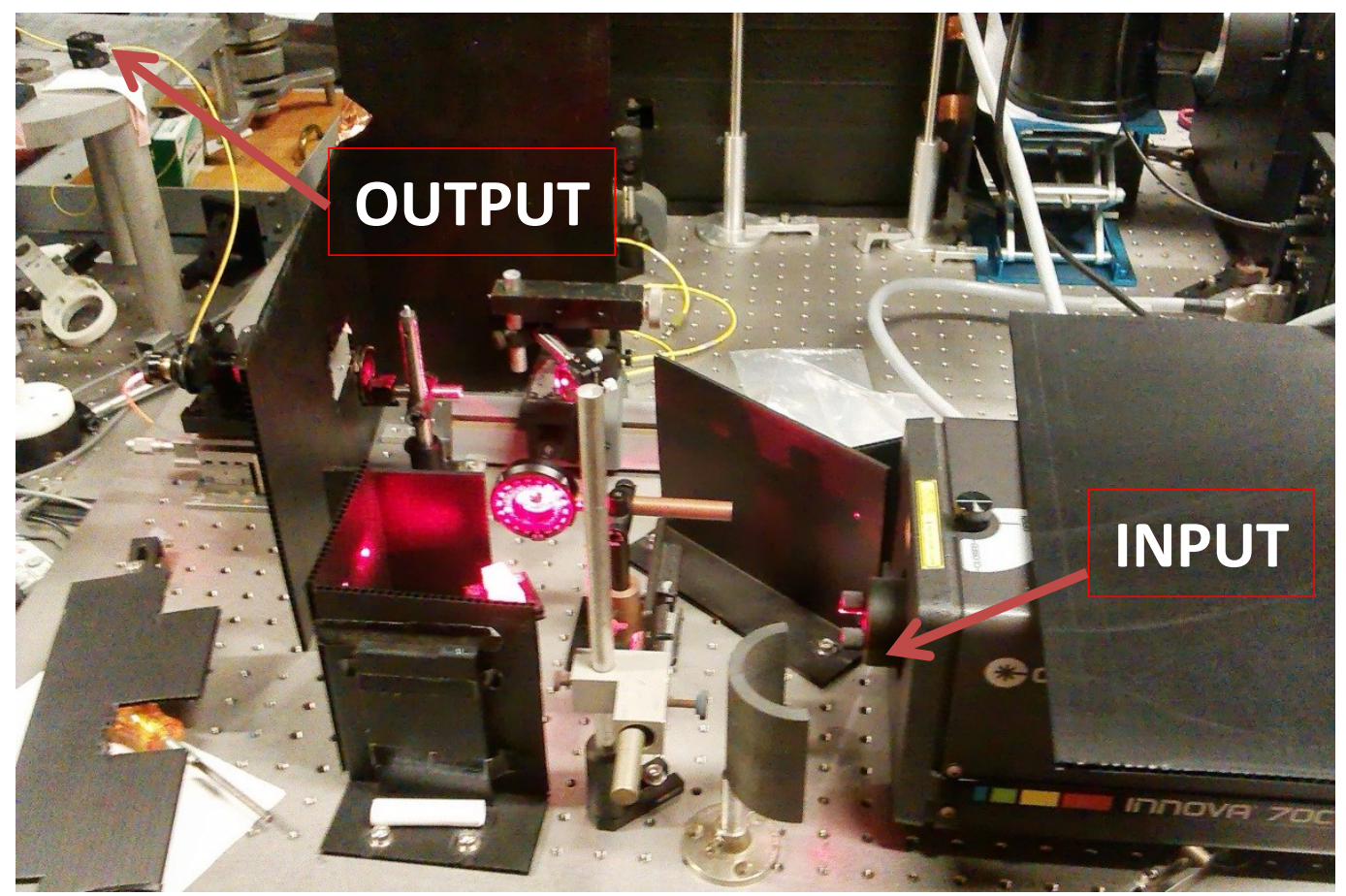

Figure 3.4.2 - The coupling apparatus is shown here. The input laser is safely directed into the fibre terminal. Upon careful adjustment, very efficient coupling is achieved, as seen in Figure 3.4.3.

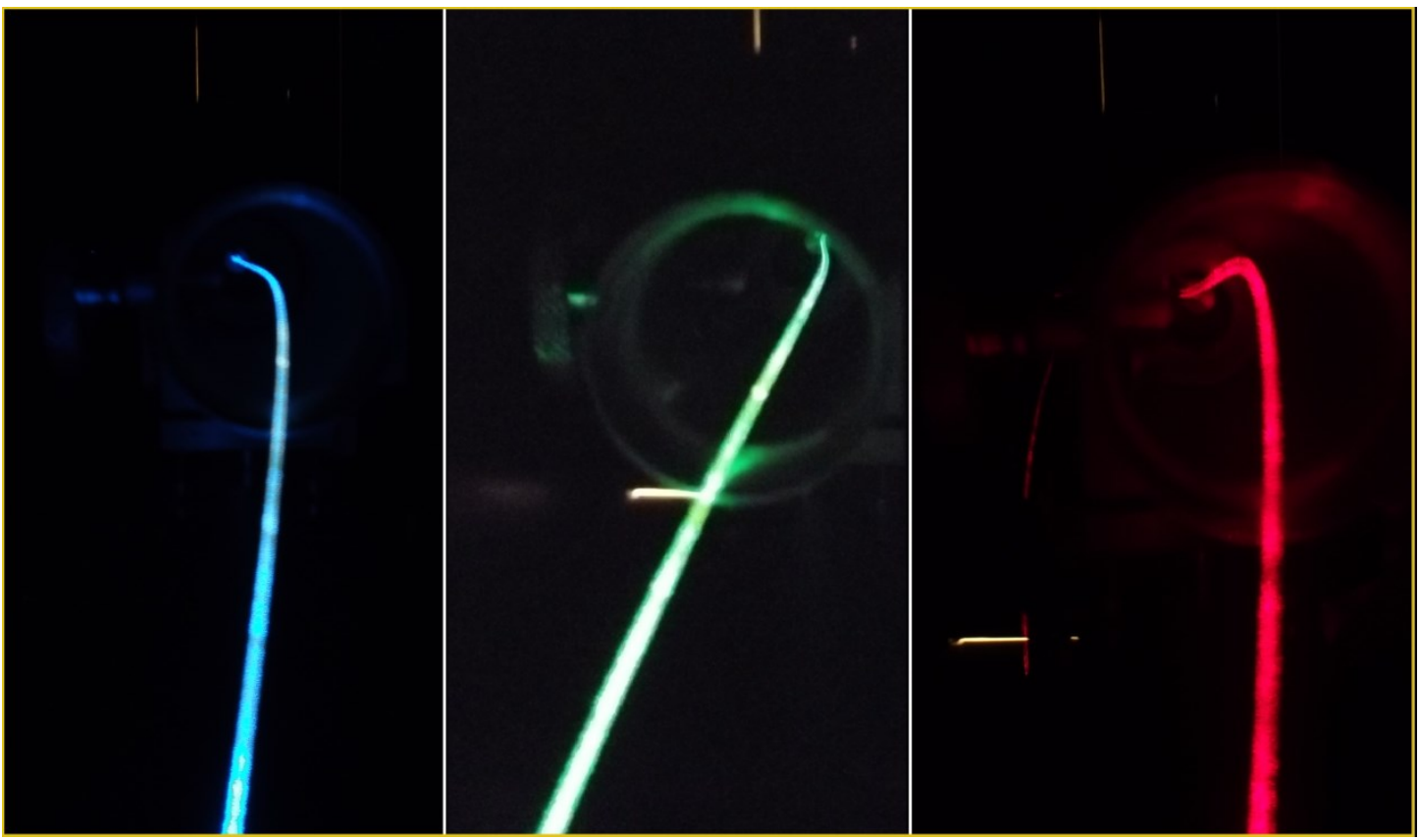

Figure 3.4.3 - After the light is coupled into the fibre, the input wavelength can simply be altered by using the digital control shown in Figure 3.4.1. Here I show the fixed end of the Goniometer (see $\mathbf{A}$ in Figure 3.3.1). The "glow" is not related to diffraction but to the scattering from multimode interference. Visible light will usually operate in multimode when traveling through standard SMF-28 fibre. 


\subsection{TFBG Fabrication - Grating Slant and Grating Pitch}

TFBGs are fabricated using the same tools and techniques as standard FBGs; from permanent refractive index changes induced in a fibre core by an interference pattern between two intense UV laser beams. There are several variants of this basic technique and they are described extensively in the literature ${ }^{5,23,24}$.

\subsubsection{Grating Slant}

Since this research is aimed at understanding visible light diffraction, I only describe here the modifications required to make "large-angle" TFBGs, which are necessary for visible outcoupling to work. These large angle gratings make it possible for some of these modes to reach the cladding, or even leave the fibre altogether. The higher the tilt angle, the more orders are observed, and the more pronounced the outcoupling effects become. See Figure 3.5.1 showing the setup used in TFBG fabrication.

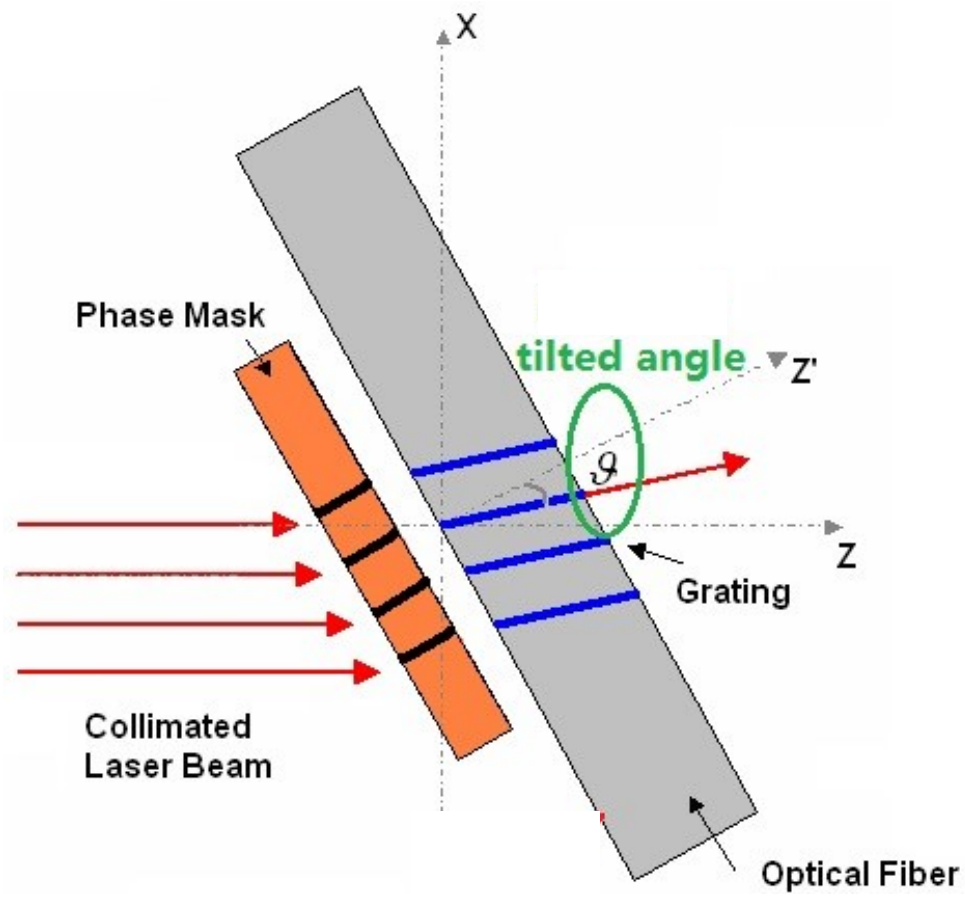

Figure 3.5.1 - TFBG fabrication setup: tilt angles reported in this research are those marked in green. 


\subsubsection{Grating Pitch}

Since the relationship between phase mask periodicity and TFBG grating pitch is discussed in Chapter 2, here I describe the relevance of testing grating pitch in the context of understanding visible light diffraction in TFBG. For a given phase mask, the fabrication setup shown in Figure 3.5.2 will control the grating slant (or tilt angle) of the fibre. However, by using different phase masks, we are able to control the grating pitch itself. This allows an additional experiment where the tilt is kept constant to isolate and test for the effects of TFBGs with different values of Bragg wavelength. Though the results of these experiments are preliminary, results in Chapter 4 show that these relationships are important since these two parameters, namely grating tilt and grating pitch, affect the output in different ways, further discussed in Chapter 5. Although drastic changes in the tilt may affect the grating pitch, within the fabrication ranges considered in this research (tilt angles between $25^{\circ}-35^{\circ}$ ), the grating pitch is assumed to remain unaffected by grating tilt.

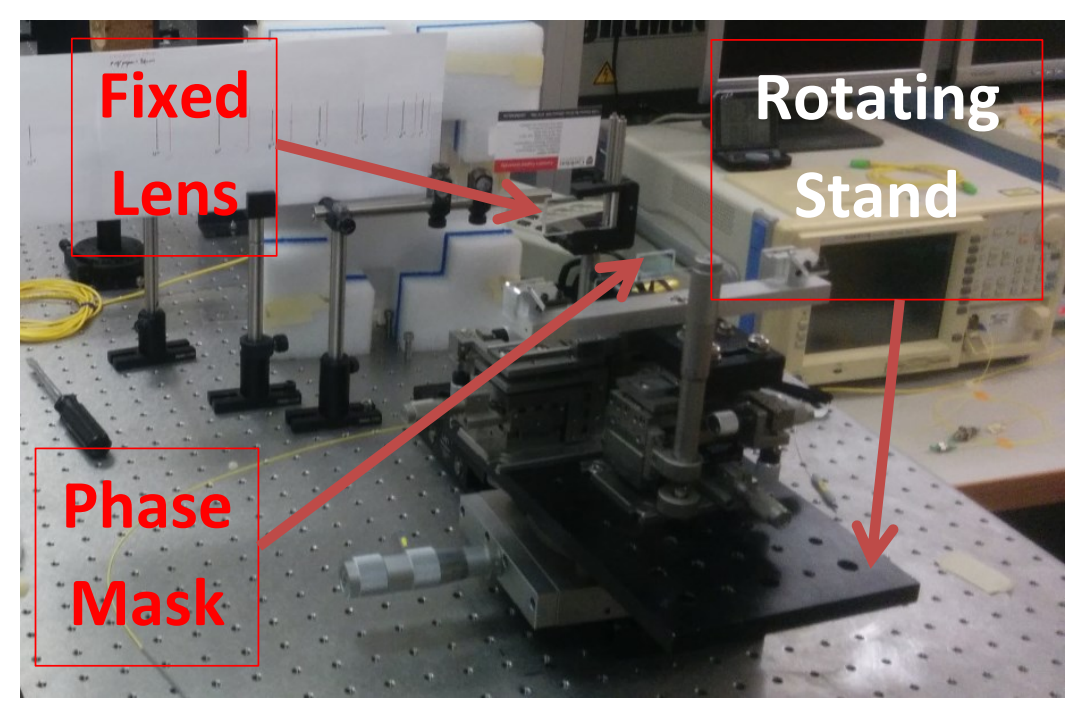

Figure 3.5.2 - In this configuration, the lens is fixed and the phase mask is rotated using the rotating stand. This setup can be used to produce gratings with tilts up to $30^{\circ}$. 


\subsubsection{Fiber Type}

Since this research was undertaken with the goal of maximising visible light outcoupling, many parameters were considered. None turned out to be as important as the type of fiber used in the inscription process. Shown in Figure 3.5.3 is a special type of cladding mode suppressed fiber (UVS-652) manufactured by a CorActive $^{\circledR}$, a specialty optical fiber manufacturer. As shown in Chapter 4, this fiber turned out to be a phenomenal test fiber for visible light outcoupling, showing drastic improvements in outcoupling efficiencies across the entire visible spectrum. These results were compared to standard SMF-28 fiber and UVS-INT fiber, another specialty type of fiber that exhibited major outcoupling, though not as pronounced as the UVS-652 shown here below.

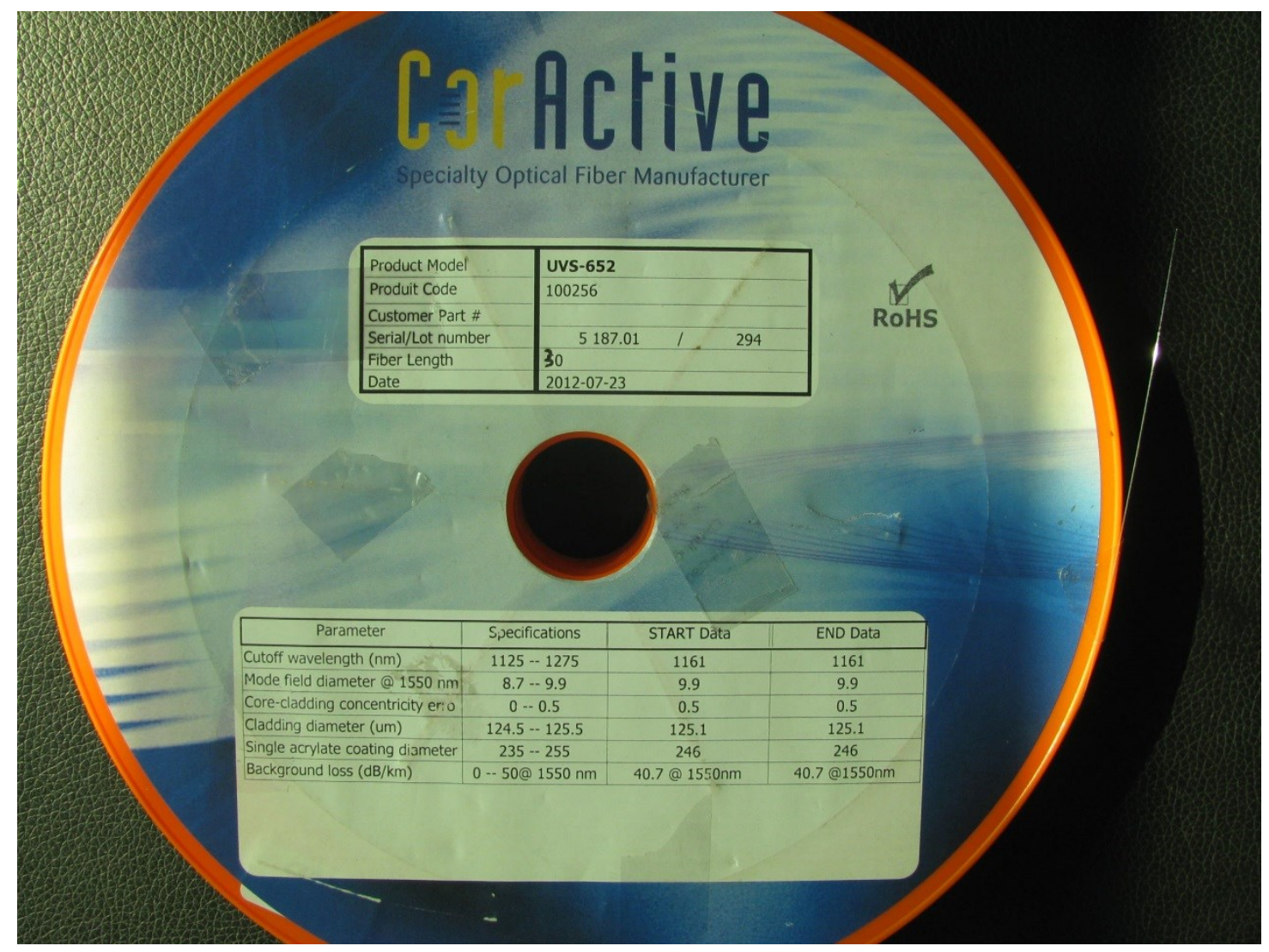

Figure 3.5.3 - UVS-652 Specialty Optical Fiber Product. Manufactured by CorActive ${ }^{\circledR}$. 


\subsection{Multimodal Theoretical Simulations Ignoring Dispersion Effects}

Since most of the experimental work in this project was in multi-mode operation (visible light in SMF-28 fiber), a computational mode solver software called FIMMWAVE ${ }^{\circledR}$ was used. This program was used to model the shapes of individual modes in order to predict the effect of single- versus multi-mode coupling. The fundamental and first harmonic modes are shown in Figure 3.6.1. The remainder of the simulations are shown in Appendix B for the sake of concision. This set of data is the modal analysis for only one m-number, from which the spectral width of a radiation peak for this m-number is measured as the sum of the modes. If the gratings were fabricated with grating pitches nearer to visible light wavelengths (400700nm) then I should expect much narrower spectral widths for individual radiation peaks, however this is a challenge yet unachieved in literature and far more work will required to fabricate single-mode visible light gratings that outcouples light at high efficiencies. It is also important to note that for the remainder of the thesis I will ignore the dispersion effects that cause higher losses in shorter wavelengths traveling in optical fibre. This is due to the very short distance (approximately 10-20 cm) traveled by incoming light before hitting the TFBG.
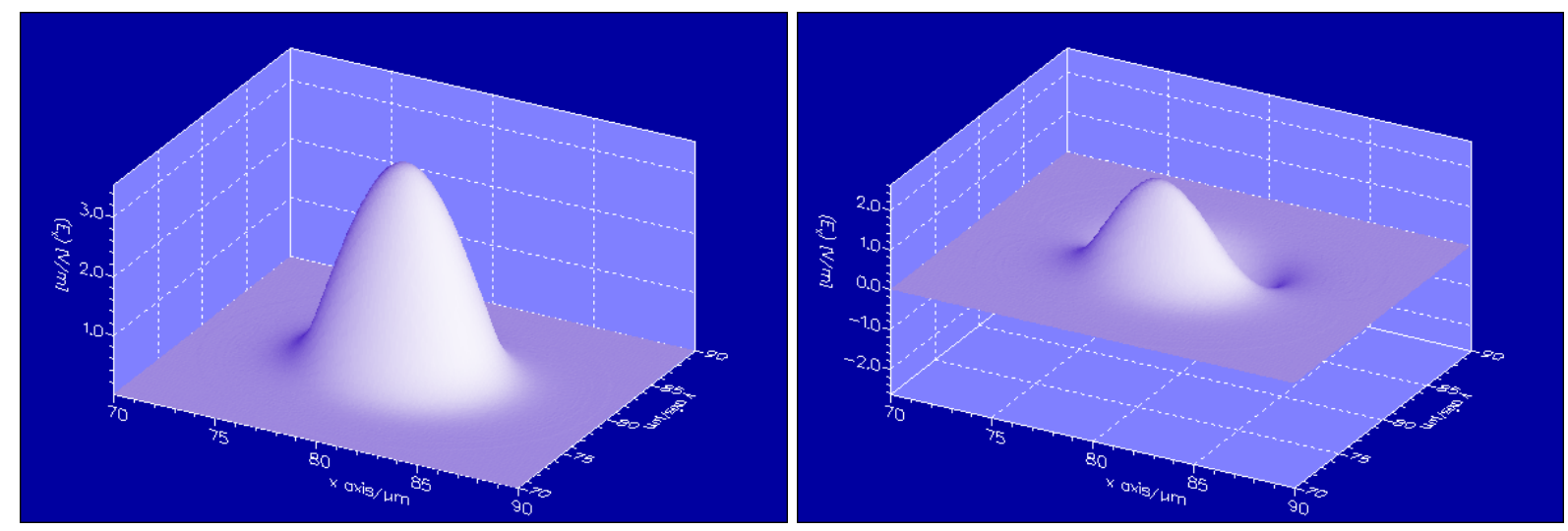

Figure 3.6.1 - Fundamental (left) and first-order (right) modes, for 632nm light traveling in SMF-28 fibre. 


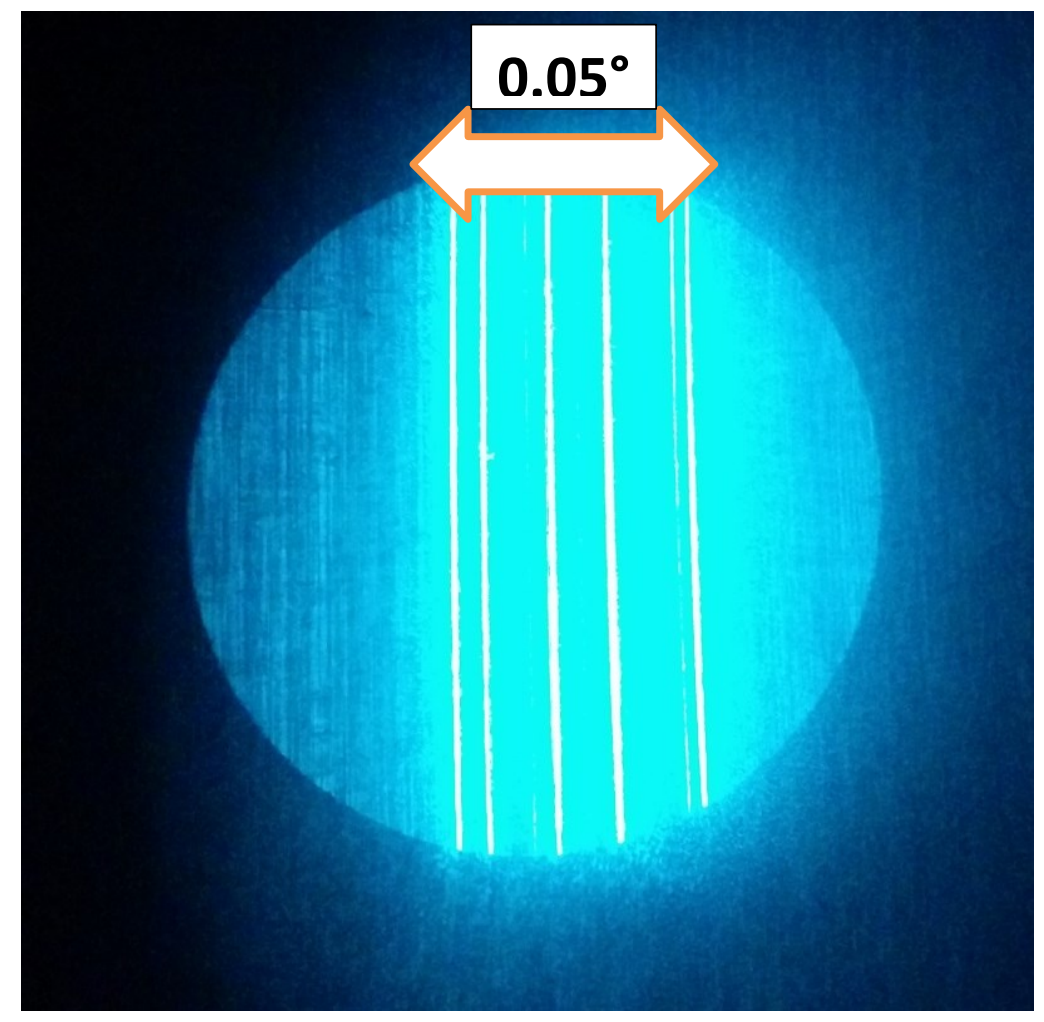

Figure 3.6.2 - A radiation profile showing spectral width of one diffracted mode. This spectral width was confirmed using FIMMWAVE ${ }^{\circledR}$ simulations with input wavelength $488.0 \mathrm{~nm}$.

This assumption should be valid, and it is also made in Kashyap's model ${ }^{17}$. The input power is adjusted at the insertion point of the light right before hitting the TFBG and the output power is measured directly from the radiated light. By minimising the effects of dispersion losses, the output efficiency is calculated in the same way for all colours tested.

To explain the angular spread of outtapped modes shown in Figure 3.6.2 one must consider the distribution characteristics of higher order modes in a multimode fiber ${ }^{19}$. A complete derivation of cladding modes power distribution is shown in Appendix A. This derivation represents the theoretical underpinning used to explain the relationship between parameters such as mode number and outcoupling behaviour. Computational simulators such as 
FIMMWAVE $^{\circledR}$ (see Appendix B) are also used to confirm that the angular spread shown in

Figure 3.6.2 is reasonable based on this relationship.

In summary, in order to validate the results later seen in this report, FIMMWAVE was used to understand the relationship between cladding modes and a broad radiation pattern observed of about $0.05^{\circ}$ (or $0.4 \mathrm{~nm}$ across) as shown in Figure 3.6.2. For ideal, single mode, operation there should theoretically be one sharp vertical line of radiation, so this image shows that this fiber supports a number of modes. Theoretically, due to the slight shifts in propagation constants of higher order modes, there would be slight shifts in the effective wavelengths of these modes as they make contact with a TFBG, which would change their exit angles slightly but measurably. The data and plots in Appendix B were extracted using FIMMWAVE, and the corresponding propagation constants were found and substituted into the phase matching equation in Section 2.5 (equation 2.5.2), predicting a spectral width of about $0.05^{\circ}$ for a $30^{\circ}$ TFBG, thus validating the span of output angles instead of just one sharp line. This effect, as mentioned, is related to the multimode operation of SMF-28 fibre in the visible region. Since higher-order cladding modes are bound to exist in such fibres at these wavelengths, the experimental observation is corroborated by the theoretical predictions. In conclusion, the observed spread makes sense according to our calculations.

\subsection{Conclusion}

Based on our findings, the phenomena of interest in visible light range do not occur until grating slant is at least $30^{\circ}$. The grating slant thus affects mainly the power but not the angle of any outcoupled light (this is explained in Section 2.5). Conversely, any variation in the grating 
Pitch strongly affects the angle of outcoupled light. In the following sections I isolate for these two parameters by explicitly showing the outcoupled radiation patterns as a function of grating slant as well as grating pitch. First I show the validation of our setup by comparing our results with theoretical predictions for various wavelengths across the visible spectrum for a TFBG slanted at $37.5^{\circ}$ and with a pitch of $512 \mathrm{~nm}$. The theoretical approach used was derived from the simple phase-matching condition shown in Chapter 2.

In this chapter I showed the original apparatus design and its validation. By implementing a validated setup for visible-light testing purposes a fundamental contribution was made toward the Advanced Photonic Components Group's broader goal of studying the interaction of both visible and IR light with TFBGs, with the ultimate purpose of improving our photonic sensors. Following the observation of near-perfect agreement between the empirical and predicted results, the validation hurdle was passed, paving the way for study of the effects of grating slant and pitch on the outcoupled radiation. The results of these effects are described in Chapter 4 and analyzed in Chapter 5. 


\section{Chapter 4}

\section{Results of Visible Diffraction Experiments: Angular and Power Distribution}

In this chapter the main body of findings of our research is presented. This body is divided into several sections. In section 4.1 I show visual results from the initial validation experiment, which confirmed the feasibility of our novel apparatus. In section 4.2, our first attempts at producing visible out-tapping were compared to reference gratings (Table 4.1.1). These reference gratings showed relatively drastic outcoupling efficiencies, and an exponential dependence of outcoupled irradiation on grating tilt. Statistical agreement is shown between phase matching predictions and empirical angular distribution values (see Appendix D).

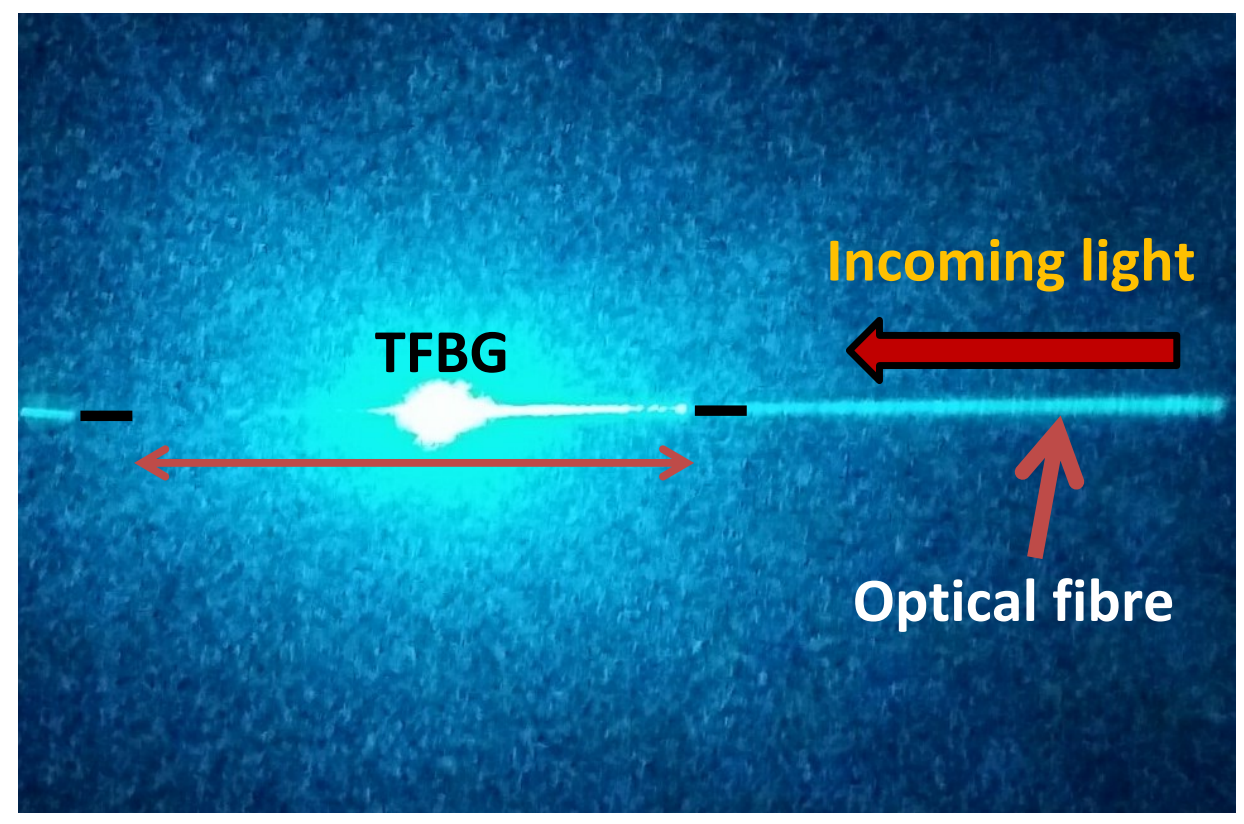

Figure 4.0.1 - Intense blue light outcoupling (white) observed for $30^{\circ} \mathrm{TFBG}$, fiber shown horizontally in blue. The radiation efficiency measured for this grating surpassed that of the TFBG-R-37.5 . 
In section 4.3, the results of reference gratings made with specialty optical fiber (see Figure 3.4.3) served as our first clue that our gratings may not have been written with optimal writing conditions. These gratings did not work efficiently due to incorrect laser orientation during fabrication, which caused incoherent TFBG fabrication. Upon rectifying these issues, an extreme improvement in outcoupled efficiencies was seen in our second generation gratings, surpassing those of the reference gratings. Finally, it is shown that in the linear optical regime the input power of light and its polarization have negligible effects on the distribution of outcoupled light. Parameter dependencies of outcoupled light on grating slant, grating pitch and input wavelength are summarized graphically. The important results of the chapter are summarized in the concluding section.

\subsection{Apparatus Feasibility and Initial Results}

When the concept of this research was devised, the first challenge was to design an apparatus capable of measuring the angular distribution of outcoupled diffraction orders as well as their respective power levels (or efficiencies). This was done by using the Goniometer described and shown in Sections 3.1-3.2. In addition, an optical power meter was used to measure input, transmitted and outcoupled power in order to calculate relative power efficiency values. The optical power meter was attached to the viewing end of the Goniometer and as it was rotated around the TFBG, the power readings were measured at regular angular intervals (see Figure 3.2.2). The results for this $10^{\circ} 1610 \mathrm{~nm}$ grating confirmed the feasibility of the apparatus and its readings, as shown in Figure 4.1.1. Azimuthal angles for HeNe were measured for validation. 
Table 4.1.1 - Type R gratings are out-of-lab-fabricated gratings that serve as references for the in-lab gratings (Types $A$ and $B$ ). *Type $A$ gratings were produced before - Type B gratings were produced after - a change was made to the fabrication setup which resulted in different behaviour.

\begin{tabular}{|c|c|c|c|}
\hline Grating Type & Fibre Type & Angles Tested & Bragg Wavelength \\
\hline \multirow{5}{*}{$\mathrm{R}$} & \multirow{5}{*}{ UVS-652 } & $20^{\circ}$ & \multirow{5}{*}{$1550 \mathrm{~nm}$} \\
\hline & & $25^{\circ}$ & \\
\hline & & $30^{\circ}$ & \\
\hline & & $35^{\circ}$ & \\
\hline & & $37.5^{\circ}$ & \\
\hline \multirow{3}{*}{$A^{*}$} & \multirow{3}{*}{ SMF-28 } & $6^{\circ}$ & \multirow{3}{*}{$1610 \mathrm{~nm}$} \\
\hline & & $10^{\circ}$ & \\
\hline & & $30^{\circ}$ & \\
\hline \multirow{3}{*}{ B } & \multirow{3}{*}{ SMF-28, UVS-INT, UVS-652 } & \multirow{3}{*}{$30^{\circ}$} & $1610 \mathrm{~nm}$ \\
\hline & & & 976nm (SMF-28 only) \\
\hline & & & 663nm (SMF-28 only) \\
\hline
\end{tabular}

Table 4.1.1 above shows the distinction between gratings used as reference, type "R" gratings, and gratings used for testing purposes, type " $A$ " and " $B$ " gratings. The reference gratings These gratings were provided by Professor Tuan GUO, from Jinan University in Guangzhou, China. They were made in Fibercore cladding mode suppressed fiber similar to the CORACTIVE 652 fiber. The rest of the gratings were fabricated in-lab. The main distinction between type $A$ and type $B$ gratings lies in the fabrication setup. Due to misalignment in the excimer laser the lower spatial coherence leads to smaller fringe patterns, which in turn forms supposed "rough" gratings instead of holographic gratings. Type A gratings are included in this thesis because understanding their abnormal behaviour is of theoretical interest. 


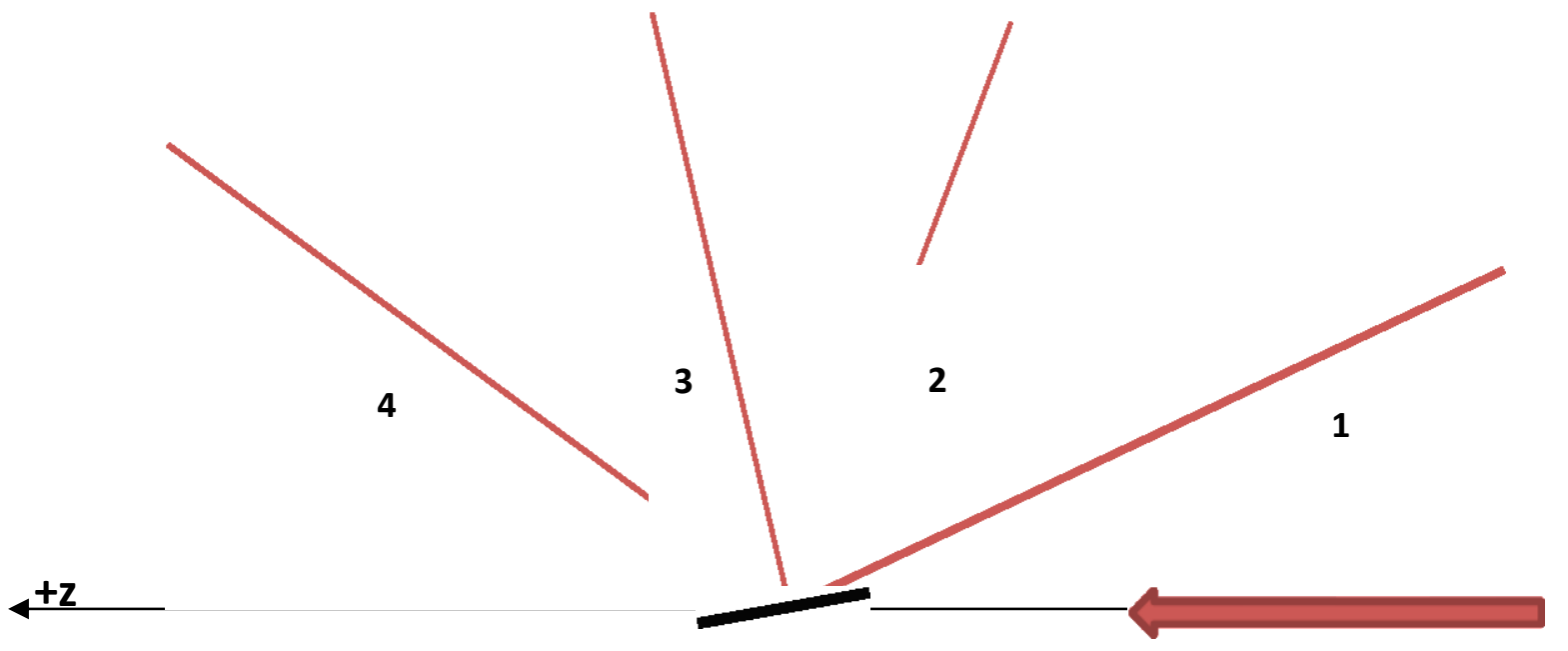

Figure 4.1.1 - Efficiency and azimuthal angles of scattered modes for sample $10^{\circ}$ grating using HeNe laser (632nm input wavelength). $\theta_{1}=25.2^{\circ}, \theta_{1}=69.4^{\circ}, \theta_{1}=102.9^{\circ}, \theta_{1}=143.6^{\circ}$ (angles referenced to negative $z$ axis direction). In this preliminary experiment, power readings were qualitative.

Three important observations were extracted from these results.

1) the apparatus was accurate enough to an error of less than $0.2^{\circ}$

This important result confirmed the feasibility of the apparatus in measuring angles, allowing further data to be acquired simply by repeating the experiment for different gratings and fibres. The power measurements were easily taken by placing a power meter at the "eye" of the Goniometer (Figure 4.1.2).

2) the angles shown in Figure 4.1.1 agreed with phase-matching predictions Even though the outcoupling efficiencies were later improved drastically, this showed an encouraging agreement between experiment and theory.

3) the diffracted mode with the largest efficiency was the forward-propagating mode The outcoupling pattern is shown in Figure 4.1.1. Although angles were consistent with phasematching, the theoretical expectation was that the backward-propagating mode should have the largest efficiency ${ }^{25}$ and that the shape should be crescent-like ${ }^{17}$. 


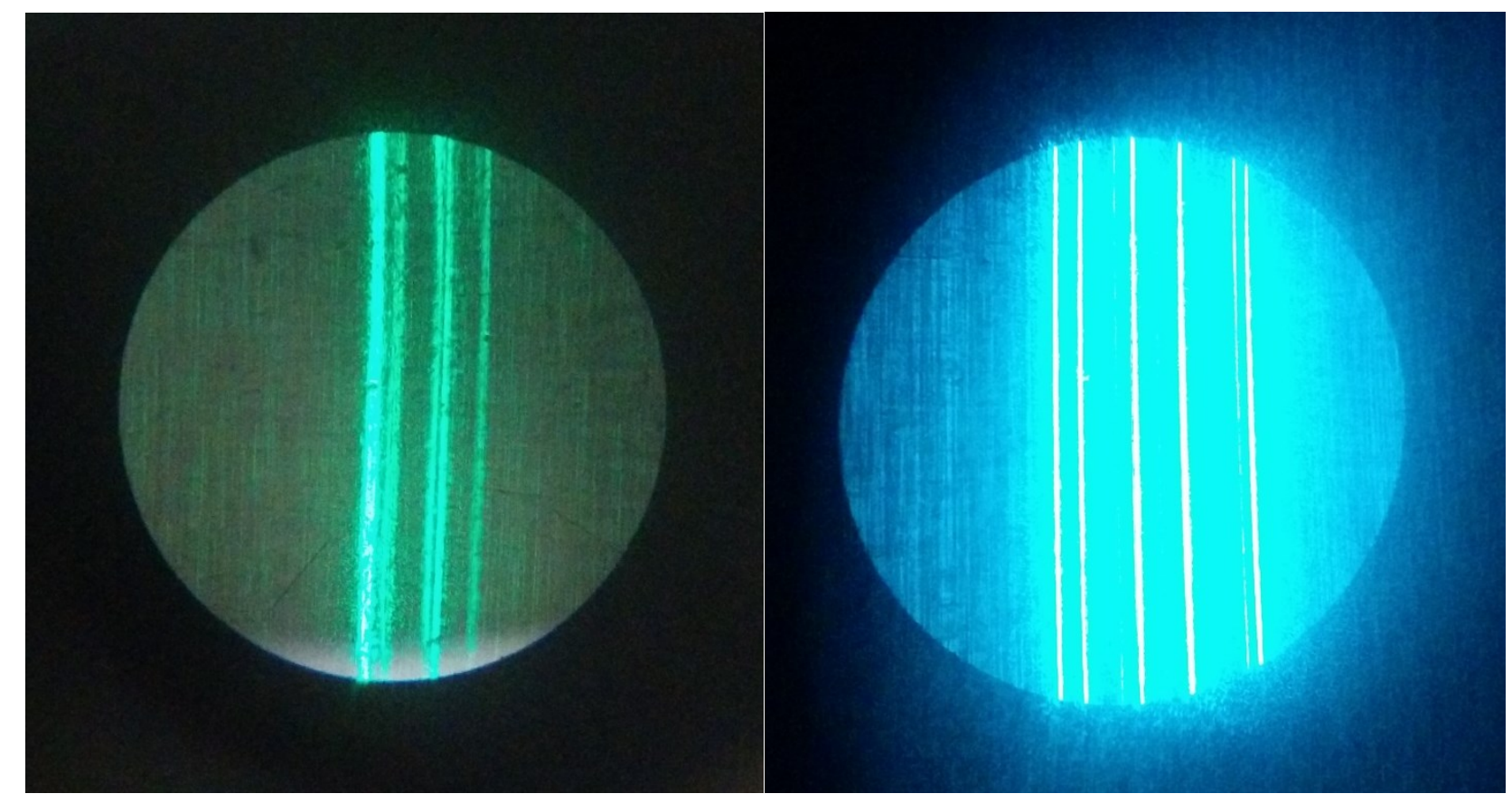

Figure 4.1.2 - Sample green $(514.5 \mathrm{~nm})$ and blue $(488.0 \mathrm{~nm})$ output radiation patterns are shown. An optical power meter was placed at in front of the slot shown, measuring the output power of each diffracted mode relative to input power.

\subsection{Phase Matching - Reference Gratings}

Since the gratings used in the preliminary tests in Section 4.1 did not agree well with theoretical predictions, more work was needed to understand whether the underlying issue was due to the gratings we had fabricated, from the apparatus itself, or from a previously unknown physical phenomenon. In order to isolate for these variables, holographic gratings were ordered and tested (TFBG-R). These gratings showed drastic improvements in two areas. First, the outcoupled power was extremely high, enough to see visible radiation several metres away on the wall in a dark room. Second, the shapes and angles of the diffracted modes agreed perfectly with theory. The crescent-like pattern reported in the literature ${ }^{17}$ was observed, while the agreement between predicted angles and observed angles are shown in Figure 4.2.1 below. Note the strong overlap between predicted (filled) and experimental values (empty). 


\subsubsection{Phase-Matching Predictions versus Observations}

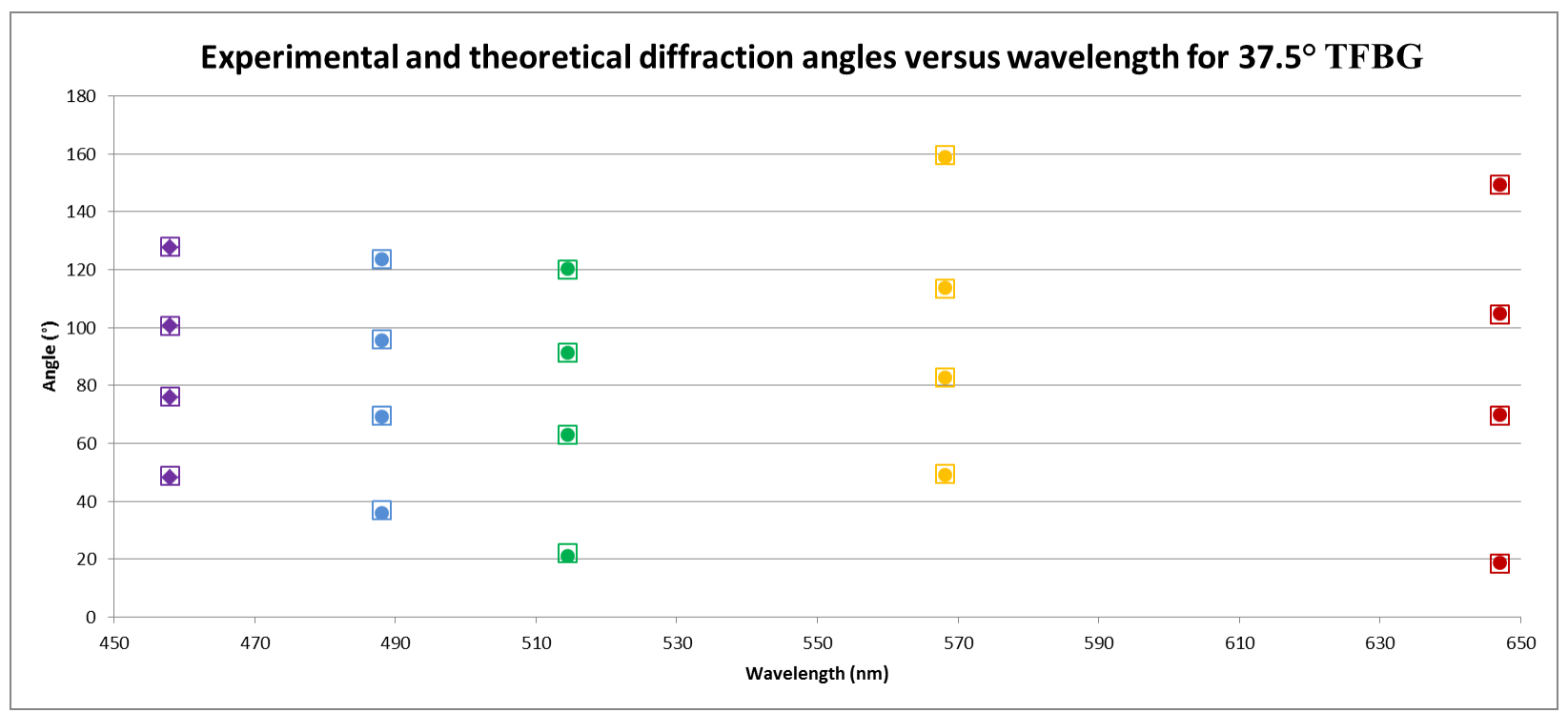

Figure 4.2.1 - The agreement is clear between predicted and observed angular distribution for several colours including purple $(457.9 \mathrm{~nm})$, blue $(488.0 \mathrm{~nm})$, green $(514.5 \mathrm{~nm})$, yellow $(568.2 \mathrm{~nm})$, red $(647.1 \mathrm{~nm})$ Predicted values are shown as filled circles, while observed values are shown as empty squares.

Figure 4.2.1 leaves no doubt as to the agreement between the phase-matching predictions of visible outcoupling in TFBG and observations made with our apparatus. Chapter $\mathbf{5}$ contains more rigorous statistical comparisons showing a Chi-square value of 0.00444 , meaning the results agree to a 95\% confidence interval. Furthermore, the variance in the observed results is $0.3^{\circ}$, which is smaller than the reading error, meaning the observed values are strongly statistically correlated and that this experiment should be easily reproducible.

\subsubsection{Relationship Between Output Efficiency and Grating Tilt}

A total of five gratings were tested as reference, ranging from $20^{\circ}$ to $37.5^{\circ}$. To track the relationship between output power and grating tilt (or slant), the backward-coupled mode was chosen and measured for each grating $\left(20^{\circ}, 25^{\circ}, 30^{\circ}, 35^{\circ}\right.$, and $\left.37.5^{\circ}\right)$ as shown in Figure 4.2.2. 
An extremely insightful result, Figure 4.2.2 shows that for grating angles greater than $30^{\circ}$, the output power displays a marked jump. This relationship makes it clear that grating tilt plays a large role in outcoupling efficiencies. Further work is required to verify whether this trend continues onward to $45^{\circ}$, or if there is an "ideal" tilt at which the outcoupled radiation peaks, however previous literature has confirmed that $45^{\circ}$ is expected to produce optimized radiation. $^{17}$

Though not shown here for the sake of brevity, all five gratings showed exactly the same angular distributions (see Figure 4.2.1) within reading errors. Therefore, for the range tested, I observed an exponential or power law relationship between grating tilt and efficiency - and a negligible relationship between grating tilt and angular distribution - of the outcoupled radiation pattern for visible light diffraction in tilted fiber Bragg gratings.

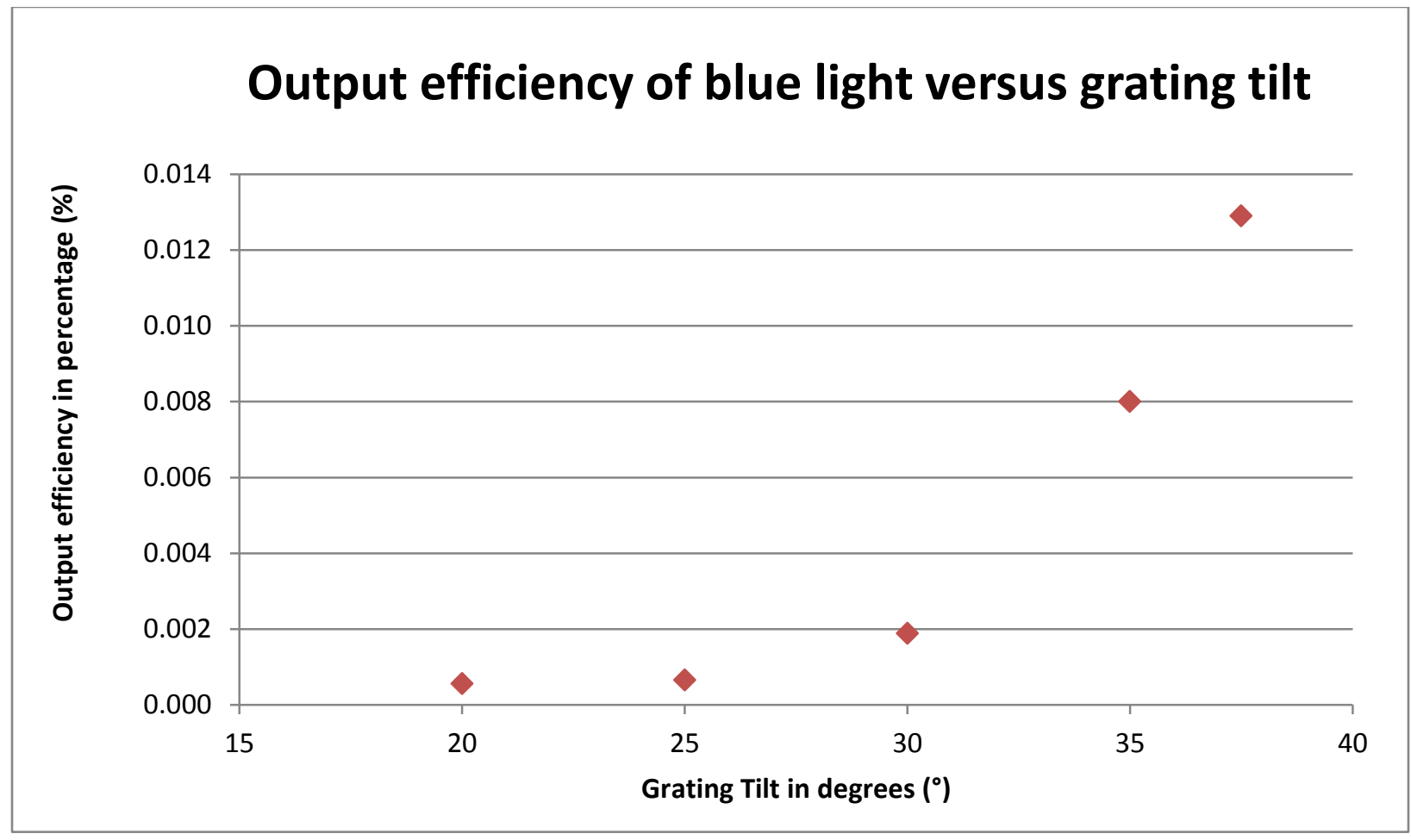

Figure 4.2.2 - Graph showing relationship between outcoupled power of backward-coupled mode versus grating angle. These results are for blue light $(488.0 \mathrm{~nm})$ for reference. 


\subsection{Large-Angle Grating Fabrication: Improved Design and Efficiency}

It was discovered that the laser orientation used in the fabrication process was sub-optimal, which would severely affect the quality of the gratings produced. Upon optimising this detail, the gratings showed behaviour consistent with Type-R gratings.

\subsubsection{Clues of Sub-Optimal Fabrication Process: "Low quality" TFBGs}

The corrections made to our gratings were a result of two separate sources of information. Initially, the first gratings tested (see Section 4.1) showed inconsistent behaviour with theoretical predictions (see Figure 4.3.1). Namely, the radiation pattern for these gratings was circular instead of crescent-like as seen for the gratings discussed in Section 4.2, and the initial gratings radiated primarily in the forward direction instead of the backward direction.

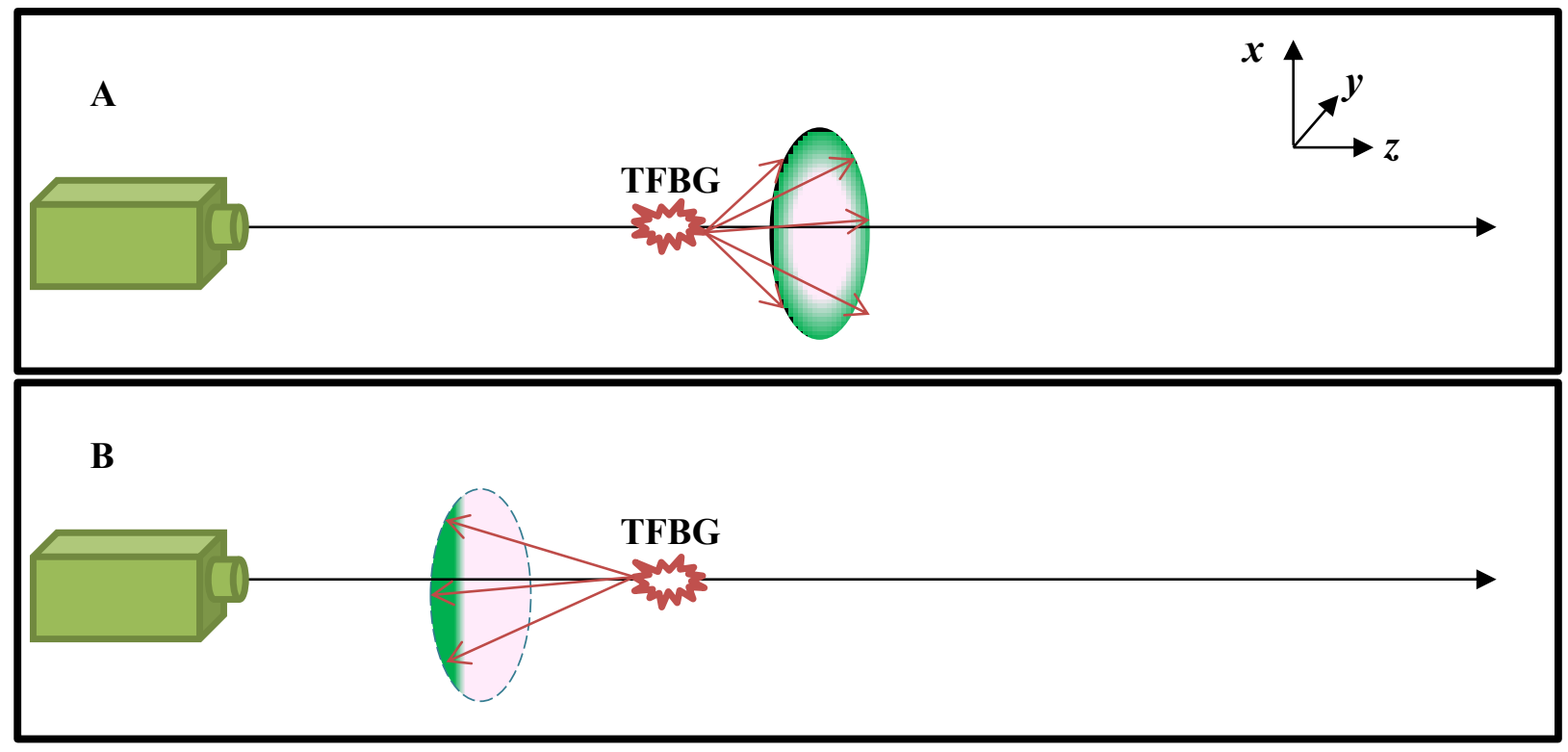

Figure 4.3.1 - A) The shape of the radiation profile was circular for the first generation of gratings, while B) the shape of the profile once corrections were made agreed with theory. Only the strongest radiation mode is shown, i.e. for A) the forward propagating mode - and for B) the backwardpropagating mode. 
Table 4.3.1 - The angles of radiation for four modes, and their respective efficiencies, are given for three gratings. Input power was fixed at $0.27 \mathrm{~W}$, efficiencies are included directly. All gratings have $30^{\circ}$ slants. Table A (top) is for green light $-514.5 \mathrm{~nm}$, and Table B (bottom) is for blue light $-488.0 \mathrm{~nm}$.

\begin{tabular}{|c|c|c|c|c|c|c|c|c|}
\hline Fibre Type & $\begin{array}{l}\theta_{1} \\
\left(^{\circ}\right)\end{array}$ & $\begin{array}{c}\varepsilon_{1} \\
(\%)\end{array}$ & $\begin{array}{l}\theta_{2} \\
\left({ }^{\circ}\right)\end{array}$ & $\begin{array}{c}\varepsilon_{2} \\
(\%)\end{array}$ & $\begin{array}{l}\theta_{3} \\
\left({ }^{\circ}\right)\end{array}$ & $\begin{array}{c}\varepsilon_{3} \\
(\%)\end{array}$ & $\begin{array}{l}\boldsymbol{\theta}_{4} \\
\left(^{\circ}\right) \\
\end{array}$ & $\begin{array}{c}\varepsilon_{4} \\
(\%)\end{array}$ \\
\hline SMF-28 & 30.0 & 0.0043 & 68.0 & 0.0001 & 95.0 & 0.0001 & 123.0 & 0.0009 \\
\hline UVS-INT & 30.7 & 0.0230 & 68.6 & 0.0003 & 95.4 & 0.0004 & 123.6 & 0.0027 \\
\hline UVS-652 & 30.0 & 0.0826 & 67.9 & 0.0012 & 94.7 & 0.0004 & 122.9 & 0.0015 \\
\hline Fibre Type & $\begin{array}{l}\theta_{1} \\
\left(^{\circ}\right) \\
\end{array}$ & $\begin{array}{c}\varepsilon_{1} \\
(\%) \\
\end{array}$ & $\begin{array}{l}\theta_{2} \\
\left({ }^{\circ}\right) \\
\end{array}$ & $\begin{array}{c}\varepsilon_{2} \\
(\%)\end{array}$ & $\begin{array}{l}\theta_{3} \\
\left(^{\circ}\right) \\
\end{array}$ & $\begin{array}{c}\varepsilon_{3} \\
(\%) \\
\end{array}$ & $\begin{array}{l}\boldsymbol{\theta}_{4} \\
\left({ }^{\circ}\right) \\
\end{array}$ & $\begin{array}{c}\varepsilon_{4} \\
(\%)\end{array}$ \\
\hline SMF-28 & 44.0 & 0.0011 & 74.0 & 0.0003 & 99.0 & 0.0004 & 127.0 & 0.0009 \\
\hline UVS-INT & 44.6 & 0.0059 & 74.5 & 0.0006 & 99.7 & 0.0004 & 127.2 & 0.0025 \\
\hline UVS-652 & 43.5 & 0.0285 & 73.4 & 0.0020 & 98.6 & 0.0012 & 126.2 & 0.0035 \\
\hline
\end{tabular}

This type of radiation is typical of surface relief gratings. Here they would result from poorly defined grating planes that would behave as omnidirectional scatterers instead of plane interfaces between regions of high and low refractive index. Those gratings were made with an incorrect orientation of the excimer laser beam.

\subsubsection{Comparing TFBGs}

As shown in Figure 4.3.1, the corrections made to the apparatus resulted in a marked change in the behaviour of visible light diffraction. Another important distinction was the drastic improvement in visible sidetapping efficiencies. In order to simultaneously account for the effect of fibre type on radiation intensity, three different fibres were used (see Table 4.1.1). The results are tabulated in Table 4.3.1 above, showing the four orders of diffraction modes for each grating and the corresponding power reading. Results for both green $(514.5 \mathrm{~nm})$ and blue (488.0nm) input light are shown. 
For red light and green light, the efficiencies of the TFBG-R-37.5 grating were very high for the backward-outcoupled mode, at $0.23 \%$ and $0.18 \%$, respectively. However, given the nature of the rise in efficiency as a function of tilt, the fact that our $30^{\circ}$ gratings displayed $0.083 \%$ efficiency for green light (nearly half the strength of TFBG-R-37.5 ${ }^{\circ}$ ) is encouraging. As shown in Figure 4.2.2, the dependence of efficiency on grating slant is shows a marked rise as the grating angle increases above $30^{\circ}$. For this reason, it is remarkable that our $30^{\circ}$ gratings showed even greater efficiencies for blue light outcoupling (shown in bold in Table 4.3.2) than even TFBG-R$37.5^{\circ}$ (see Figure 4.2.2). The exact reason for this improvement in efficiencies between our Type $B$ gratings and Type $R$ gratings may be explained by the small difference in Bragg wavelength (1610nm for Type B, 1550nm for Type R), however more work is required to test this hypothesis. Pictures were taken of both green and blue outcoupled radiation to visually confirm these results, shown in Figure 4.3.2 below.

\subsection{Input Power, Polarization, Wavelength and Grating Pitch}

In order to address the effects of input power, polarization, wavelength and grating pitch on output radiation, an experiment was devised to measure the efficiency and angular distribution of outcoupled radiation as a function of these parameters. It is shown that both input power and polarization have negligible impacts on angular distribution, while more work may be required to fully understand their effects on efficiency in the nonlinear regime. The pitch of the grating, which determines the Bragg wavelength of the TFBG, was used as a control parameter to address and further validate the phase-matching analysis in the context of fibre grating taps for visible light. Observations of the effect of input wavelength are also shown. 


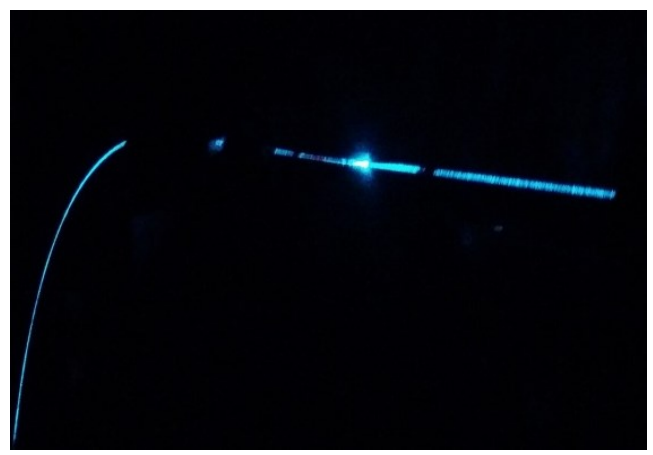

0.0285\% Efficiency

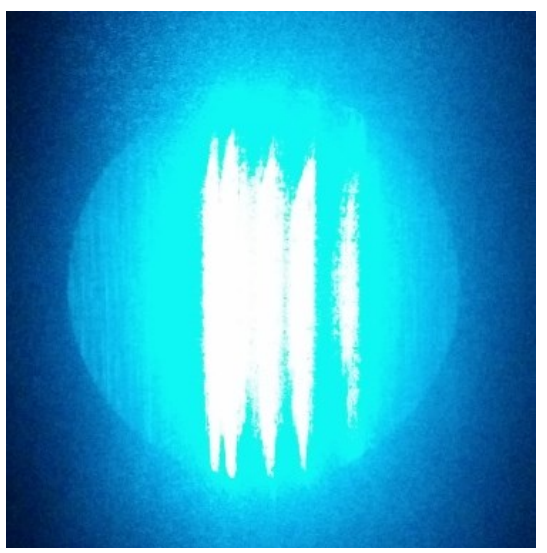

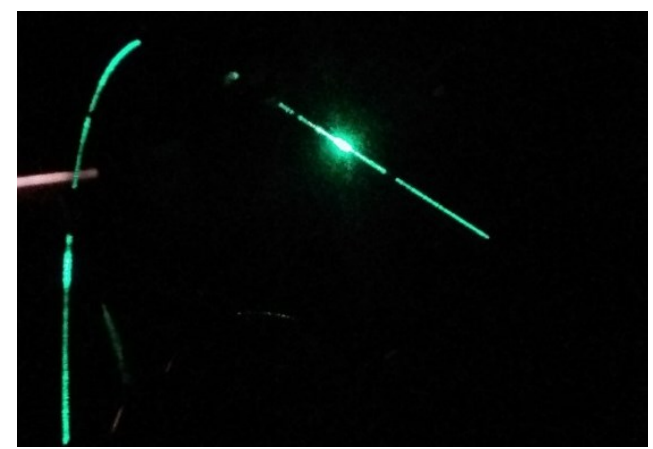

$0.0826 \%$ Efficiency

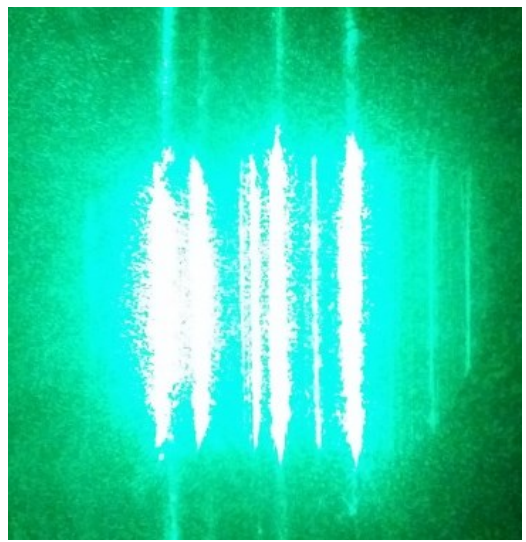

Figure 4.3.2 - Strong visible outcoupling of UVS-652 $1 \mathrm{~cm} 30^{\circ} 1610 \mathrm{~nm}$ TFBG. Shown on top are pictures taken from afar (intense outcoupling at TFBG in centre) and below through a spectroscope. It is important to note that these pictures represent the $m=1$ diffraction mode order only. The multimodal nature of the guided radiation results in several lines showing up for each $m$ number. The lines shown in these pictures do NOT represent different $m$ numbers, only the spectral width of one m-number due to multimode operation of visible wavelengths in a 1610nm TFBG.

\subsubsection{Independence of Angular Distribution on Input Power and Polarization}

The polarization of the incoming light was adjusted using a standard polarization rotator (see

Figure 4.4.1). Polarization was adjusted in increments of $5^{\circ}$ for a complete $360^{\circ}$ perspective. This experiment showed that the angular distribution of outcoupled light for all gratings tested (including type $A$, type $B$ and type $R$ gratings) was unaffected by polarization. This results establishes that, for the samples observed, the angular distribution of outcoupled radiation is independent of input polarization. Input power was varied in increments of $0.05 \mathrm{~mW}$ from $0.05 \mathrm{~mW}$ to $3.00 \mathrm{~mW}$ and then in increments of $10 \mathrm{~mW}$ from $10 \mathrm{~mW}$ to $270 \mathrm{~mW}$ to test its effect 
on angular distribution. The result of this experiment showed no correlation, which indicates that angular distribution of outcoupled radiation is independent of input power. Indeed, neither the angular distribution nor the radiation profiles were affected by either input power or polarization. In the nonlinear regime, input power could affect mode distribution so these results confirm that the experiments conducted in this research were in the linear regime.

\subsubsection{Effect of Input Power and Polarization on Sidetapping Efficiency}

From section 4.4.1, it is clear that input polarization has no considerable effect on either output efficiency or radiation profiles. However, it is important to note that future work could include further control of the polarization in such a way that the directionality of the output

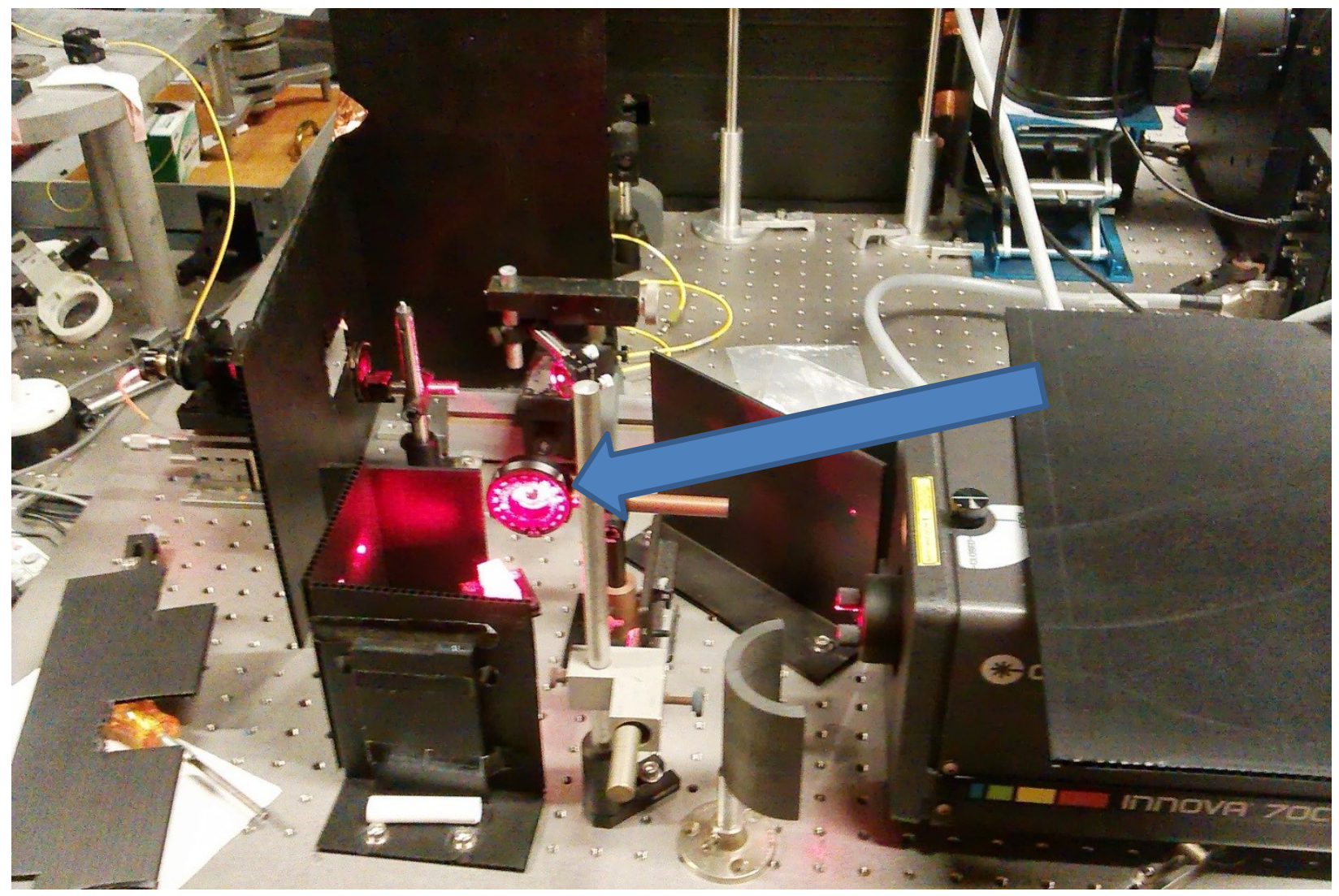

Figure 4.4.1 - Polarization rotator was incrementally adjusted to test effect on outcoupled radiation. Results showed that both output efficiency and angular distribution are independent of polarization. 
Table 4.4.1 - The angles of radiation for four modes, and their respective efficiencies for sample grating $\left(37.5^{\circ}\right)$. Input power was not fixed, values are shown for each colour. Efficiencies are calculated directly.

\begin{tabular}{|c|c|c|c|c|c|c|c|c|c|}
\hline $\begin{array}{c}\text { Input } \\
\text { Power }\end{array}$ & $\begin{array}{c}\text { Input } \\
\text { Wavelength }\end{array}$ & $\begin{array}{c}\boldsymbol{\theta}_{\mathbf{1}} \\
\left({ }^{\circ}\right)\end{array}$ & $\begin{array}{c}\boldsymbol{\varepsilon}_{\mathbf{1}} \\
(\%)\end{array}$ & $\begin{array}{c}\boldsymbol{\theta}_{\mathbf{2}} \\
\left(^{\circ}\right)\end{array}$ & $\begin{array}{c}\boldsymbol{\varepsilon}_{\mathbf{2}} \\
(\%)\end{array}$ & $\begin{array}{c}\boldsymbol{\theta}_{\mathbf{3}} \\
\left({ }^{\circ}\right)\end{array}$ & $\begin{array}{c}\boldsymbol{\varepsilon}_{\mathbf{3}} \\
(\%)\end{array}$ & $\begin{array}{c}\boldsymbol{\theta}_{4} \\
\left({ }^{\circ}\right)\end{array}$ & $\begin{array}{c}\boldsymbol{\varepsilon}_{4} \\
(\%)\end{array}$ \\
\hline $430 \mathrm{~mW}$ & $647.1 \mathrm{~nm}$ & 21.6 & $\mathbf{0 . 1 0 4 7}$ & 69.6 & 0.0033 & 104.5 & 0.0052 & 149.4 & 0.0116 \\
\hline $360 \mathrm{~mW}$ & $568.2 \mathrm{~nm}$ & 49.3 & 0.0015 & 82.6 & 0.0002 & 113.5 & 0.0004 & 159.5 & 0.0010 \\
\hline $400 \mathrm{~mW}$ & $514.5 \mathrm{~nm}$ & 23.0 & $\mathbf{0 . 1 2 0 0}$ & 62.9 & 0.0004 & 91.3 & 0.0002 & 120.0 & 0.0005 \\
\hline $450 \mathrm{~mW}$ & $488.0 \mathrm{~nm}$ & 37.0 & 0.0129 & 69.4 & 0.0003 & 95.8 & 0.0003 & 123.7 & 0.0008 \\
\hline $100 \mathrm{~mW}$ & $457.9 \mathrm{~nm}$ & 48.0 & 0.0005 & 76.1 & 0.0001 & 100.6 & 0.0002 & 127.8 & 0.0003 \\
\hline
\end{tabular}

may be controlled. Using a sample grating (TFBG-R-35) efficiencies for the backward-coupled mode were measured to be roughly the same (between $0.009-0.010 \%$ ) for input power levels ranging from $0.1 \mathrm{~W}$ to $0.43 \mathrm{~W}$ for $647.1 \mathrm{~nm}$ (red) input radiation. The range in these values is well within the error of the power readings, which was calculated to be $\pm 0.002 \%$. Thus our results indicate sidetapping efficiency is negligibly affected by input power and polarization.

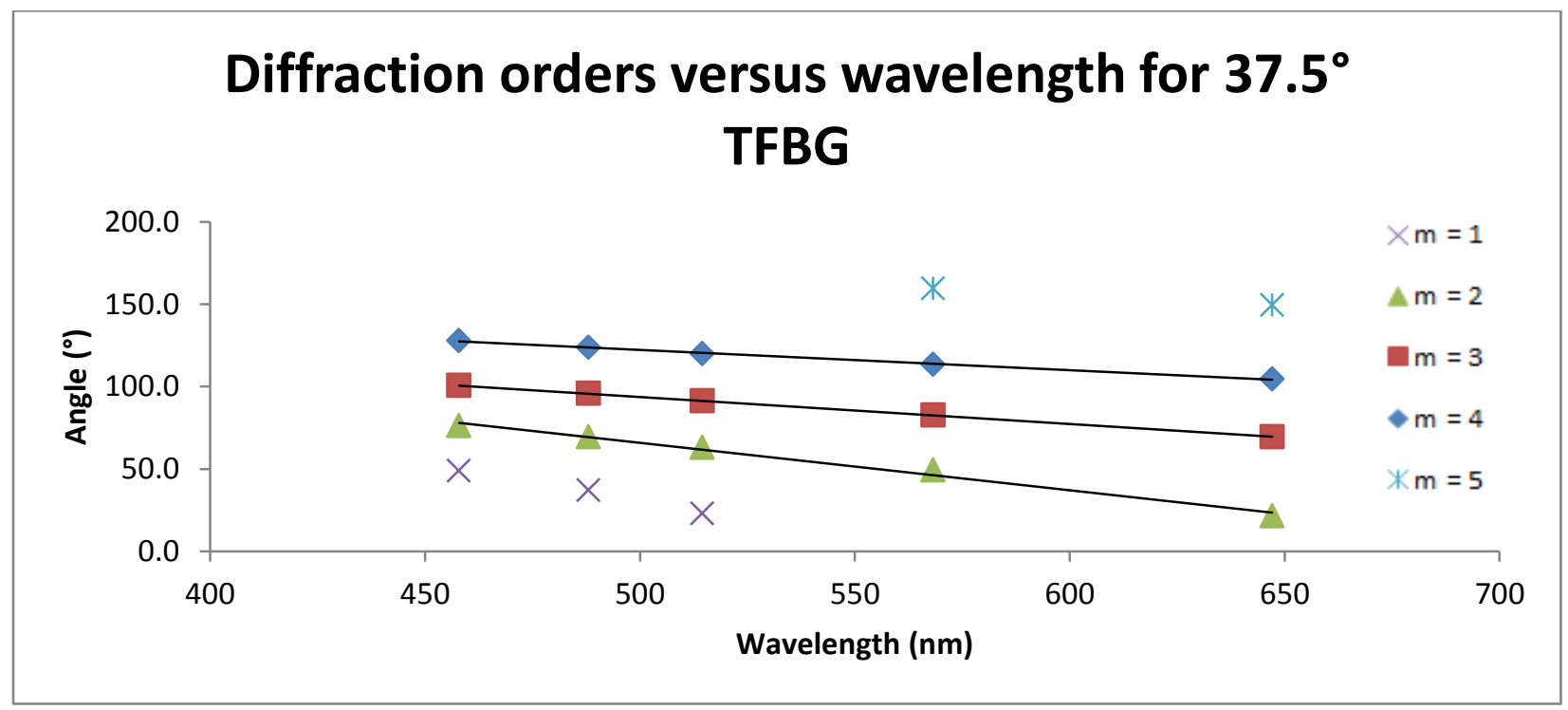

Figure 4.4.2 - The angles of outcoupled radiation are shown and collected into similar modes. That is, each mode is shown changing slightly as the wavelength is altered, exhibiting a linearly decreasing relationship to increasing wavelength. This can be verified by the phase-matching equation. 
Table 4.4.2 - The angles of radiation, experimental and theoretical, for the backward-sidetapped mode for various wavelengths of a $976 \mathrm{~nm}$ grating. Only theoretical values are shown for the $663 \mathrm{~nm}$ grating.

\begin{tabular}{|c|c|c|c|}
\hline \multirow{2}{*}{ Grating Pitch } & Input Wavelength & $\begin{array}{c}\text { Theoretical } \boldsymbol{\theta} \\
\left({ }^{\circ}\right)\end{array}$ & $\begin{array}{c}\text { Experimental } \boldsymbol{\theta} \\
\left({ }^{\circ}\right)\end{array}$ \\
\hline \multirow{2}{*}{$976 \mathrm{~nm}$} & $568.2 \mathrm{~nm}$ & 47.2 & 45.5 \\
\cline { 2 - 4 } & $514.5 \mathrm{~nm}$ & 40.7 & 38.4 \\
\cline { 2 - 4 } & $488.0 \mathrm{~nm}$ & 37.3 & 35.0 \\
\hline \multirow{2}{*}{$663 \mathrm{~nm}$} & $568.2 \mathrm{~nm}$ & 67.8 & $\mathrm{NA}$ \\
\cline { 2 - 4 } & $514.5 \mathrm{~nm}$ & 61.3 & NA \\
\cline { 2 - 4 } & $488.0 \mathrm{~nm}$ & 58.0 & NA \\
\hline
\end{tabular}

Using a sample grating (TFBG-R-37.5 $)$, all four orders of visible outcoupled light were measured for angles as well as power efficiency and the results are shown in Table 4.4.1 above and Figure 4.4.2 below. Angular distributions have a linear dependence on mode number.

\subsubsection{Effect of Grating Pitch}

To begin accounting for the effect of grating pitch on output radiation, three gratings with different Bragg wavelengths were chosen and tested. These gratings had Bragg wavelengths of $1610 \mathrm{~nm}, 976 \mathrm{~nm}$ and $663 \mathrm{~nm}$, respectively. Results agree weakly with phase-matching analysis.

The results for the $1610 \mathrm{~nm}$ have been extensively reported in Section 4.3 , so I discuss here only those for the $976 \mathrm{~nm}$ and $663 \mathrm{~nm}$ gratings. All gratings tested were in SMF-28 fiber. Both gratings showed significantly reduced outcoupling efficiencies and it was observed that the 663nm TFBG did not produce any outtapping at all. However, future work specialty optical fibres may yet produce different results and this is indeed part of the research path for this project. Therefore, only the experimental results for the $976 \mathrm{~nm}$ grating are reported in Table 
4.4.2 above, which shows the experimental and theoretical values of angular distribution for the main backward-sidetapped mode for $976 \mathrm{~nm}$, while also showing the theoretically expected angular values for the $663 \mathrm{~nm}$ grating in the absence of experimental data.

\subsection{Conclusion}

To summarize the salient points of this chapter, our results indicate that simple phasematching predictions are compatible with our experimental results, showing strong agreement. This indicates the success of our apparatus to empirically extract the angular distribution profiles of various TFBG configurations. The versatility of this apparatus is its most attractive feature, since it can easily isolate and thus compare the effects of many input variables including input power, polarization and wavelength as well as grating pitch while measuring output variables in a systematic way, including output power, angular distribution of diffracted radiation as well as azimuthal, radial and axial radiation profiles.

We are encouraged by the stark improvement in efficiencies and the progress made in the short span of this thesis work, and with our current understanding of the importance of photosensitive fibres it would be interesting to retest the effects of grating pitch to fabricate TFBGs that operate in single mode in the visible region. This should improve efficiencies even further. Finally, it would be necessary to produce $45^{\circ}$ gratings at some point, as this angle is expected to maximize radiation efficiency. 


\section{Chapter 5}

\section{Analysis and Significance of Angular and Power Distribution Results}

The main results are emphasized in this chapter and their significance in the context of future research is discussed. The results from the previous Chapter make it clear that the research of visible light sidetapping from Tilted Fibre Bragg Gratings has interesting research potential. In the short span of a 20-month thesis project, much progress was made in understanding the various components that affect and govern outcoupling radiation. The fabrication method of TFBG, the type of fibre used, the grating pitch, the grating tilt and the input wavelength will all affect the output radiation profile, which can be broken into relationships between input variables and output, namely:

a) the angular distribution of diffracted modes (see Figure 4.1.1 and Figure 4.3.1) and

b) the power or efficiency of outcoupled radiation, as represented in Figure 4.2.2 and Tables 4.3.1 and 4.4.1.

The main goal of this future research is in understanding and controlling blue light sidetapping for novel sensing solutions in DNA- and biomolecular-detection applications.

\subsection{Fabrication Techniques}

Although the first set of gratings made in Advanced Photonic Components Group (APCG) laboratory were "low quality" gratings (labeled TFBG-A in Table 4.1.1), this allowed us to study the effects of 
varying the TFBG fabrication setup. As shown in Figure 4.3.1, these weak gratings completely changed the expected output characteristics of diffracted light. The salient points distinguishing these gratings from 'good' gratings are that:

a) the angular distribution remained unaffected, but the main diffraction mode was forward-coupled, not backward-coupled (as observed for TFBG-B gratings),

b) the outcoupled efficiency was so weak as to render the outcoupling nearly impossible to measure (even the forward-coupled mode), and

c) the radiation profile, as shown in Figure 4.3.1, was circular, instead of crescent-shaped.

Points b) and c) above are actually quite interesting and the physical phenomenon underpinning this behaviour could be useful to understand exactly what is happening in the fibre as a grating is fabricated. What happens to the grating inside the fibre that so drastically alters the output behaviour? Can this behaviour be controlled and optimized after all?

Regardless, once the corrections to the excimer laser orientation were made, further comparisons between different types of optical fiber were investigated as described in Sections 3.4 and 4.3. To control the grating tilt, the rotating setup shown below could be adjusted while the focusing lens was kept fixed (see green arrows in Figure 5.1.1) until a grating reached a desired tilt. As far as limitations, the largest angles that could be achieved using this fabrication setup were $30^{\circ}$ gratings. Gratings shown in Section 4.3 were made using this technique. Preliminary work explored the option of rotating the lens in parallel with the rotating stage to attempt even larger angle gratings (see blue arrows in Figure 5.1.1), but so far these efforts have proven fruitless. In conclusion, the first technique worked best - shown in green in Figure 5.1.1 - and more work is required to produce even larger angle gratings. 


\subsubsection{Power Optimisation - Effect of Grating Tilt}

In order to optimize sidetapping efficiency, it is important to produce even larger-tilt gratings as discussed in the literature ${ }^{17}$ and corroborated by our own results in Figure 4.2.2. These results seem to show an exponential dependence of sidetapping efficiency on grating tilt angle; one of the more significant outcomes of this research. More work is required to investigate whether this exponential trend continues as grating tilt approaches $45^{\circ}$ or if efficiency levels off. Either way it suffices to have shown that for gratings above $25^{\circ}-30^{\circ}$, the trend definitely shows a drastic improvement in efficiency therefore, regardless of input wavelength, any attempt to optimize outcoupling should begin with the fabrication of $45^{\circ}$ TFBGs.

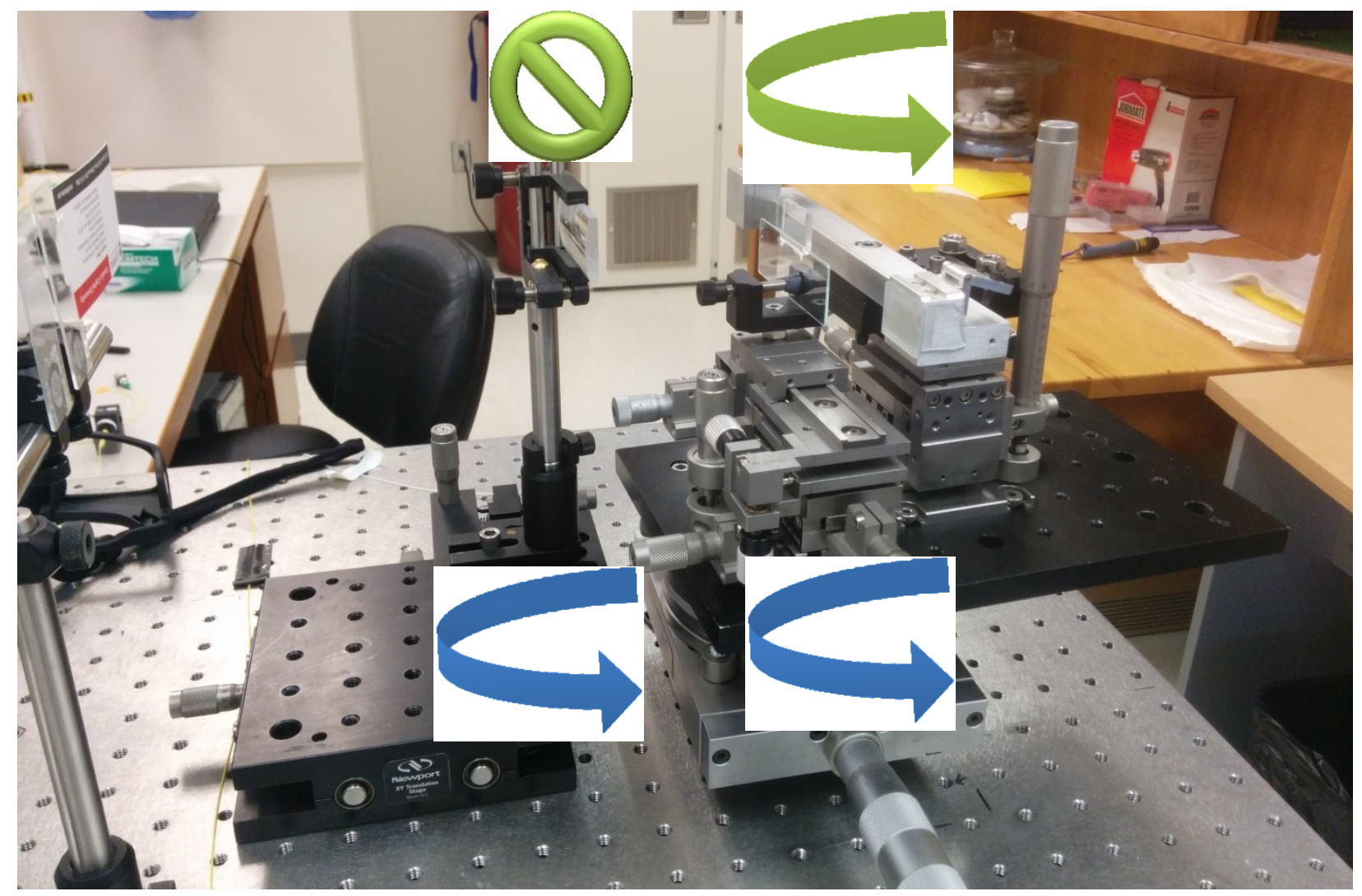

Figure 5.1.1 - The setup holding the phase mask (right) was rotated to allow fabrication of up to $30^{\circ}$ gratings, while the lens in the middle was tested in two configurations -1 ) fixed (green), and 2) rotated in parallel with the rotating plate (blue). It was found that the first setup was more feasible. 


\subsubsection{Power Optimisation - Effect of Grating Pitch}

I know from phase-matching predictions, which have been validated in the course of our research, that grating pitch strongly affects output power. In Section 4.4.4, I showed that for $30^{\circ}$ gratings with varying grating pitch, that the outcoupled power efficiency was significantly reduced. However, much more work is required to comprehensively study the effect of grating pitch on visible sidetapping. This future work is important for two reasons. First, repeated experiments may yet show significant if not improved sidetapping when photosensitive fibres are used (see Section 4.3). Second, if successful, these gratings should yield perfect or nearperfect single mode operation in the visible spectrum. This would be a huge advantage in optimising the scattered radiation by controlling both efficiency and output angle. Furthermore, this would introduce new possibilities hitherto unexplored, such as systems that could exploit polarization-selectivity. Much potential lies in this type of sensor if significant control over output radiation is achievable.

\subsubsection{Power Optimisation - Effect of Fibre Photosensitivity}

Though well explored in Section 4.3.1, it is useful to reiterate the main result: fibre photosensitivity in the cladding plays a major role in optimising output power. Comparing SMF28 fibres and UVS-652 fibres alone, Table 4.3.1 showed that photosensitivity in the cladding results in an increase in efficiency by a factor of 30 for blue light, and by factor of 20 for green light. These are very significant results especially since the main goal of this research was maximising the efficiency of blue light sidetapping. It is highly encouraging that only three types of fibre were compared; SMF-28, UVS-INT and UVS-652. 
Table 5.2.1 - Theoretical exit angles for blue light radiation for $1610 \mathrm{~nm}, 976 \mathrm{~nm}$ and $663 \mathrm{~nm}$ TFBGs..

\begin{tabular}{|c|c|c|}
\hline $\begin{array}{c}\text { Grating Pitch } \\
(\mathrm{nm})\end{array}$ & $\begin{array}{c}\text { Input Wavelength } \\
(\mathrm{nm})\end{array}$ & $\begin{array}{c}\text { Theoretical } \boldsymbol{\theta} \\
\left({ }^{\circ}\right)\end{array}$ \\
\hline 663 & 488.0 & 58.0 \\
\hline 976 & 488.0 & 37.3 \\
\hline 1610 & 488.0 & 43.5 \\
\hline
\end{tabular}

\subsection{Controlling Angular Distribution}

There are only two ways to control the scattering angles of visible light diffraction in TFBG: adjusting grating pitch, or adjusting input wavelength. In the context of this thesis work, we are primarily interested in blue light outcoupling, leaving only grating pitch as the technique of choice. If a certain exit angle is chosen, phase-matching predictions allow the calculation of an "ideal" grating pitch that should yield such radiation. For example, in Table 5.2.1 I compare the theoretical output angles of blue light $(488.0 \mathrm{~nm})$ for three gratings tested in this research. I note, however, that the relationship between grating pitch and scattered mode is not linear so any attempt to 'control' scattering angles should be very carefully studied. The reason this is not straight-forward is that more work is required to study the impact, if any, of scattering angle on blue-light sensing properties. For instance, although grating pitch clearly affects both scattering angle as well as multimode operation in the visible EM spectrum, it may be impossible to achieve a perfect balance and a trade-off may be necessary.

It is noted that although grating tilt does affect output efficiency, as summarized in Section 5.1.1, grating tilt does not affect the angular distribution profiles of outtapping. This result is clear from a phase-matching standpoint and simplifies any future work by limiting the focus to grating pitch alone. 


\subsection{Conclusion}

The most encouraging aspects of this thesis research are the accurate prediction of coupling angles from phase matching analysis, and the strong outcoupling of visible light traveling through a TFBG especially in photosensitive optical fibre. The main significance of this work is in its simple approach, which proved to be flexible and versatile. The simplicity of the apparatus described in Chapter 3 gave way to the myriad results in Chapter 4, culminating with the first reported blue light outcoupling for a relatively low-tilt grating (i.e. without using a $45^{\circ}$ TFBG).

How can we extend the knowledge gathered in this Master's thesis? What future work is required to address the limitations and restrictions encountered? Why is it important to pursue this research? Can this research be integrated into modern sensing systems to enhance current technologies, or branched out and used to produce completely novel ones?

The following chapter will attempt to summarize the main body of findings of this research work, and to answer some of these questions while discussing the context for future work on fibre grating taps for visible light. 


\section{Chapter 6}

\section{Discussion and Future Work}

At the beginning of this undertaking, the literature had shown some interest in visible outcoupling but as of yet no publicly available findings has confirmed or reported successful blue light outcoupling. In this respect, this thesis work has gone above and beyond expectations by successfully achieving intense blue light radiation from large angle TFBGs produced in-lab at the Advanced Photonic Components research group at Carleton University.

Nonetheless, including time constraints, certain limitations meant that particular areas were left unexplored. For example, there may be many benefits to shifting from multimode to single mode operation such as potentially increased power efficiency, improved sensitivity as well as polarization selectivity. Also, it is recommended by this author that a priority is placed on

future research to explore in more depth the effect of grating pitch on outcoupling properties, whether for blue light or other visible wavelengths. This would allow a build-up of empirical knowledge on the subject, which could eventually be summarized into a seminal understanding of visible light behaviour in optical fibres, SMF-28 or other, in general.

An increased understanding of the output power and angular distribution relationships to inputs such as grating tilt, grating pitch, input power, input wavelength, and azimuthal, axial and radial parameters could lead to completely novel solutions to sensing applications. However, it may be impossible or at least unfeasible to empirically test for all these parameters, which is why this author recommends an extensive effort to simulate the theoretical behaviour 
of the outcoupling behaviour of visible light. Although the angular distribution has been quite carefully understood and validated using simple phase-matching analysis in the course of this research, it is especially important to further our understanding, empirically and theoretically, on the output power behaviour of visible light as a function of the aforementioned variables.

As has been mentioned repeatedly in this document, it is essential for optimisation of outcoupled power to produce $45^{\circ} \mathrm{TFBGs}$, regardless of the pitch or other input parameters. This has been reported in the literature ${ }^{17}$ and any future expansion of this research will require this necessary step to improve outcoupling efficiency even further.

The drastic improvement in efficiency seen using photosensitive fibres as opposed to standard SMF-28 fibre is highly encouraging, especially since only two photosensitive fibres were tested (UVS-INT and UVS-652). If more research could be done on exploring a wider range of photosensitive fibres, even greater improvements in visible light efficiencies may yet be seen.

Finally, as a result of time limitations, no testing of these results directly into sensing properties was implemented. It is especially interesting that blue-light and the commonly used Ag nanocoatings in our laboratory could be combined to produce novel applications, as Ag is known to have a strong absorption peak at around $480 \mathrm{~nm}$. This is why it is paramount that the results of this and future research be applied directly to novel sensors, in order to validate their usefulness and exploit their potential in the future growth of research on the optimisation of visible light diffraction in tilted fibre Bragg gratings. 


\section{Bibliography}

1. Y. Shevchenko, G. Camci-Unal, D.F. Cuttica, M.R. Dokmeci, J. Albert and A. Khademhosseini, "Surface Plasmon Resonance Fiber Sensor for Real-Time and Label-Free Monitoring of Cellular Behaviour," Biosens. Bioelectron. 56 (2014)

2. Y. Shevchenko, T.J. Francis, D.A.D. Blair, M.C.DeRosa and J. Albert, "In situ Biosensing With a Surface Plasmon Resonance Fiber Grating Aptasensor," Anal. Chem. 83 (2011)

3. A. Bialiayeu, A. Bottomley, D. Prezgot, A. lanoul, and J. Albert, "Plasmon-enhanced refractometry using silver nanowire coatings on tilted fibre Bragg gratings," Nanotechnology 23 (2012)

4. A. Bialiayeu, C. Caucheteur, N. Ahamad, A. lanoul, and J. Albert, "Self-optimized Metal Coatings for Fiber Plasmonics by Electroless Deposition," Opt. Exp. 19 (2011)

5. Kalli, A. O. (1999). Fiber Bragg Grating, fundamentals and applications in telecommunications and sensing. House Publishers. Boston: Artech House Publisher.

6. Meltz, Morey, and Glenn "Formation of Bragg gratings in optical fibers by a transverse holographic method," Opt. Lett. 15 (1989)

7. Meltz et al. "Design and performance of bidirectional fibre Bragg grating", OSAOFC $^{\circledR}(1991)$

8. J. Albert, S. Lepinay, C. Caucheteur, and M.C. DeRosa, "High resoluition gratingassisted surface plasmon resonance fiber optic biosensor," Invited, Methods (2013)

9. T.K. Gayloard and M.G. Moharam, "Planar Dielectric Diffraction Theories," App. Phys. B 28 (1982)

10. M. Kuznetsov and H.A. Haus, "Radiation Loss in Dielectric Waveguide Structures by the Volume Current Method", J. Quant. Elect. 10 (1983)

11. Y. Li, M. Forggatt and T. Erdogan, "Volume Current Method for Analysis of Tilted Fiber Gratings," J. Light. Tech. 10 (2001)

12. C. Olson, D.G. Hall, "Radiation Patterns of Higher Azimuthal Order Spatial Modes from a Concentric-Circle-Grating Waveguide Cavity Using the Volume-Current Method," J. Quant. Elect. 33 (1998) 
13. Y. Li et al, "Influence of the longitudinal mode field in grating scattering from weakly guided optical fiber waveguides," Opt. Lett. 29 (2004)

14. S. Ramachandran and M.F. Yan, "Static and tunable dispersion management with higher order mode fibers," J. Opt. Fiber. Commun. Rep. 3 (2006)

15. R.B. Walker, S.J. Mihailov, D.G. Grobnic, P. Lu and X. Bao, "Direct evidence of tilted Bragg grating azimuthal radiation mode coupling mechanisms," Opt. Lett. 17 (2009)

16. M.J. Holmes, R. Kashyap, R. Wyatt, "Physical Properties of Optical Fiber Sidetap Grating Filters: Free-Space Model," J. Quant. Elect. 5 (1999)

17. L. Kotacka, J. Chauve, R. Kashyap, "Angular and azimuthal distribution of side scattered light from fiber Bragg gratings," Invited, Photonics North (2004)

18. M.J. Holmes, R. Kashyap, R. Wyatt, and R.P. Smith, "Ultra narrow-band optical fibre sidetap filters," ECOC (1998)

19. Lu Yan, R.A. Barankov, P. Steinvurzel, and S. Ramachandran, "Side-tap modal channel monitor for mode division multiplexed (MDM) systems," OFCC (2012)

20. R. Falciai, M. Morganti, C. Trono, "Fibre Bragg grating location and spatial period determination in DBR lasers by side-scatter detection of pump radiation," Elect. Lett. 41 (2005)

21. R.R. Maier, J.S. Barton, J.D. Jones, "Fiber Bragg grating location by a side-scatter technique based on cladding-mode." Appl. Opt. 43 (2004)

22. Chun Yang, "A novel method to measure modal power distribution in multimode fibers using tilted fiber bragg gratings," IEEE Phot. Tech. Lett. 17 (2005)

23. J. Albert, L.Y. Shao and C. Caucheteur, "Tilted Fiber Bragg Grating Sensors," Laser Photonic. Rev. 7 (2013)

24. M. Gagne, P. Sharma, R. Kashyap, "New UV Laser Sources for Fiber Bragg Grating Fabrication," WFOPC (2011)

25. Tamir, T., (1975). "Integrated Optics." Springer-Verlag, Berlin and New York (p. 98) 


\section{Appendix A: Derivation of Cladding Modes Power Distribution}

To find the coupling efficiency from the forward propagating core mode to backward coupled cladding modes in a TFBG, one must consider the power distribution characteristics that the blazing of the grating planes exhibits. The guided power in a multimode fiber can be expressed as follows ${ }^{22}$ :

$$
P_{G, m}=\sum_{i=1}^{N_{m}} P_{G, m, i}
$$

Radiation power is similarly defined:

$$
P_{R, m}=\sum_{i=1}^{N_{m}} P_{R, m, i}
$$

The relation between resonant (or degenerated) mode groups (DMG) and guided power can be represented by the following relation:

$$
\sum_{i=1}^{N_{m}} P_{G, m, i}=\sum_{i=1}^{N_{m}} K_{m, i} P_{R, m, i}
$$

where $P_{G, m}$ and $P_{R, m}$ are the guided and radiation power of the $m^{\text {th }}$ DMG, respectively, and $P_{G, m, i}$ and $P_{R, m, i}$ are the $i^{\text {th }}$ degenerated mode (DM) in the $m^{\text {th }} \mathrm{DMG}$, and $K_{m, i}$ is the reciprocal of the coupling coefficient for the $i^{\text {th }}$ DM. The coupling coefficient for a specific mode is defined as:

$$
K_{m}=\frac{\sum_{i=1}^{N_{m}} K_{m, i} P_{R, m, i}}{P_{R, m}}
$$

The total power of all the guided modes or essentially the total coupled power in the fiber of input light before reaching the TFBG is given by:

$$
P_{t}=\sum_{i=1}^{N_{m}} P_{G, m}=\sum_{i=1}^{N_{m}} K_{m} P_{R, m}
$$




\section{Appendix A: Derivation of Cladding Modes Power Distribution}

We define the fraction of power coupled into a specific cladding mode $m$ as the modal power reflection coefficient, or coupling strength, through:

$$
R_{m, d B}=-10 \log \left(\frac{P_{R, m}}{P_{t}}\right)
$$

In order to find this coupling strength within a TFBG we consider that the grating tilt is equivalent to introducing a spatially dependent phase $\Phi(r, \varphi, \theta)$ to the wavefunction $\Psi(r, \varphi)$ in the grating with uniform refractive index modulation of $\widetilde{\Delta n}$. At small tilt angles, the RI modulation is:

$$
\Delta n=\widetilde{\Delta n} \cos \left[\frac{2 \pi z}{\Lambda}+\Phi(r, \varphi, \theta)\right]
$$

where

$$
\Phi(r, \varphi, \theta)=\frac{2 \pi r \cos \varphi \tan \theta}{\Lambda}
$$

Since the fundamental core mode has only radial dependence, $\Psi(r)$ will be used instead of $\Psi(r, \varphi)$ to represent this mode. We represent the modal field distributions with $\Psi_{l m}(r, \varphi)$. The coupling strength between the core mode and the modal distribution is described by first considering the effective refractive index modulation:

$$
\widetilde{\Delta N}=\int_{0}^{2 \pi} \int_{0}^{\infty} \widetilde{\Delta n} \Psi(r) \Psi(r, \varphi) e^{j \Phi} r d r d \varphi
$$

We define an overlap integral:

$$
O L=\int_{0}^{2 \pi} \int_{0}^{\infty} \Psi(r) \Psi(r, \varphi) e^{j \Phi} r d r d \varphi
$$




\section{Appendix A: Derivation of Cladding Modes Power Distribution}

So equation (A.7) becomes:

$$
\widetilde{\Delta N}=\widetilde{\Delta n} \cdot O L
$$

The peak reflection amplitude at a wavelength $\lambda$ is then given by:

$$
R=\tanh ^{2}(k l)
$$

where $I$ is the grating length and

$$
k=\frac{\pi \widetilde{\Delta N}}{\lambda}
$$

This effective index modulation can thus be used to measure the relative coupling strength between the core mode and resonant modes. 


\section{Appendix B: FIMMWAVE ${ }^{\circledR}$ Simulations Used For Spectral Width Validation}

\begin{tabular}{|r|c|c|c|c|c|c|c|}
\hline mode & neff & 10 & 20 & 25 & 30 & 35 & 37.5 \\
\hline 1 & 1.462654 & 78.39 & 78.04 & 77.78 & 77.46 & 77.09 & 76.89 \\
\hline 2 & 1.462654 & 78.39 & 78.04 & 77.78 & 77.46 & 77.09 & 76.89 \\
\hline 3 & 1.461392 & 78.38 & 78.03 & 77.77 & 77.45 & 77.08 & 76.87 \\
\hline 4 & 1.461390 & 78.38 & 78.03 & 77.77 & 77.45 & 77.08 & 76.87 \\
\hline 5 & 1.461390 & 78.38 & 78.03 & 77.77 & 77.45 & 77.08 & 76.87 \\
\hline 6 & 1.461388 & 78.38 & 78.03 & 77.77 & 77.45 & 77.08 & 76.87 \\
\hline 7 & 1.459772 & 78.37 & 78.02 & 77.75 & 77.43 & 77.06 & 76.86 \\
\hline 8 & 1.459772 & 78.37 & 78.02 & 77.75 & 77.43 & 77.06 & 76.86 \\
\hline 9 & 1.459769 & 78.37 & 78.02 & 77.75 & 77.43 & 77.06 & 76.86 \\
\hline 10 & 1.459769 & 78.37 & 78.02 & 77.75 & 77.43 & 77.06 & 76.86 \\
\hline 11 & 1.459264 & 78.37 & 78.01 & 77.75 & 77.43 & 77.06 & 76.85 \\
\hline 12 & 1.459264 & 78.37 & 78.01 & 77.75 & 77.43 & 77.06 & 76.85 \\
\hline 13 & 1.457870 & 78.36 & 78.00 & 77.74 & 77.42 & 77.05 & 76.84 \\
\hline 14 & 1.457870 & 78.36 & 78.00 & 77.74 & 77.42 & 77.05 & 76.84 \\
\hline 15 & 1.457143 & 78.35 & 77.99 & 77.73 & 77.41 & 77.04 & 76.83 \\
\hline 16 & 1.457142 & 78.35 & 77.99 & 77.73 & 77.41 & 77.04 & 76.83 \\
\hline 17 & 1.457141 & 78.35 & 77.99 & 77.73 & 77.41 & 77.04 & 76.83 \\
\hline 18 & 1.457141 & 78.35 & 77.99 & 77.73 & 77.41 & 77.04 & 76.83 \\
\hline
\end{tabular}

Figure B.1 - Table showing mode number (see below for visual representation - not to be confused with m number), effective index for each mode, and the outcoupling angle for each mode for gratings between $10^{\circ}$ and $37.5^{\circ}$, where the tilt is shown at the top of the table. This table shows that for all value of tilt, the expected "spread", or variation in angle, for one $\mathrm{m}$ number (in this case $\mathrm{m}=2$ and wavelength of $632 \mathrm{~nm}$ ) is about $0.05^{\circ}$. 
Appendix B: FIMMWAVE ${ }^{\circledR}$ Simulations Used For Spectral Width Validation
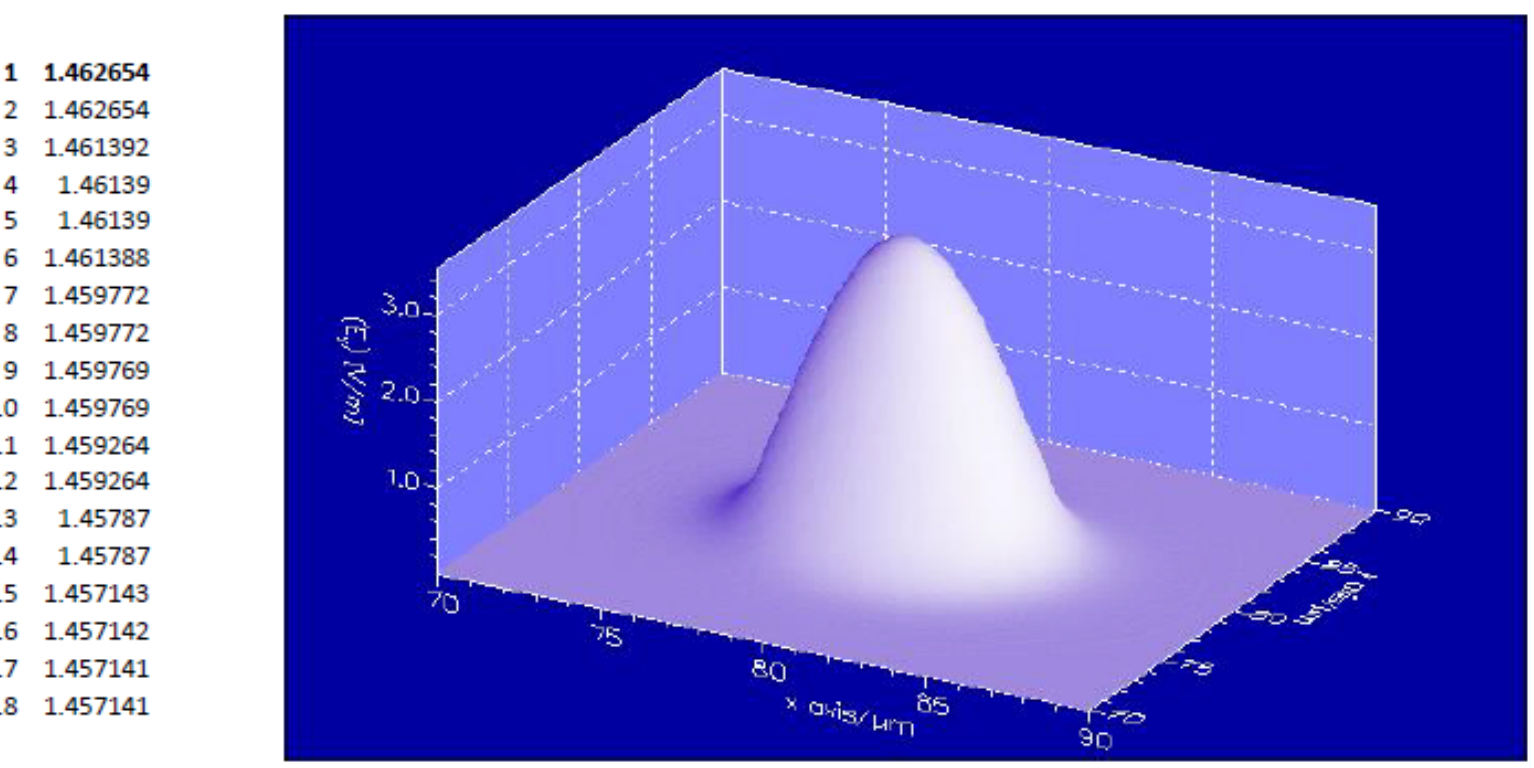

11.462654

21.462654

31.461392

$4 \quad 1.46139$

$5 \quad 1.46139$

$6 \quad 1.461388$

$7 \quad 1.459772$

81.459772

91.459769

$10 \quad 1.459769$

$11 \quad 1.459264$

$12 \quad 1.459264$

$13 \quad 1.45787$

$14 \quad 1.45787$

$15 \quad 1.457143$

$16 \quad 1.457142$

$17 \quad 1.457141$

$18 \quad 1.457141$

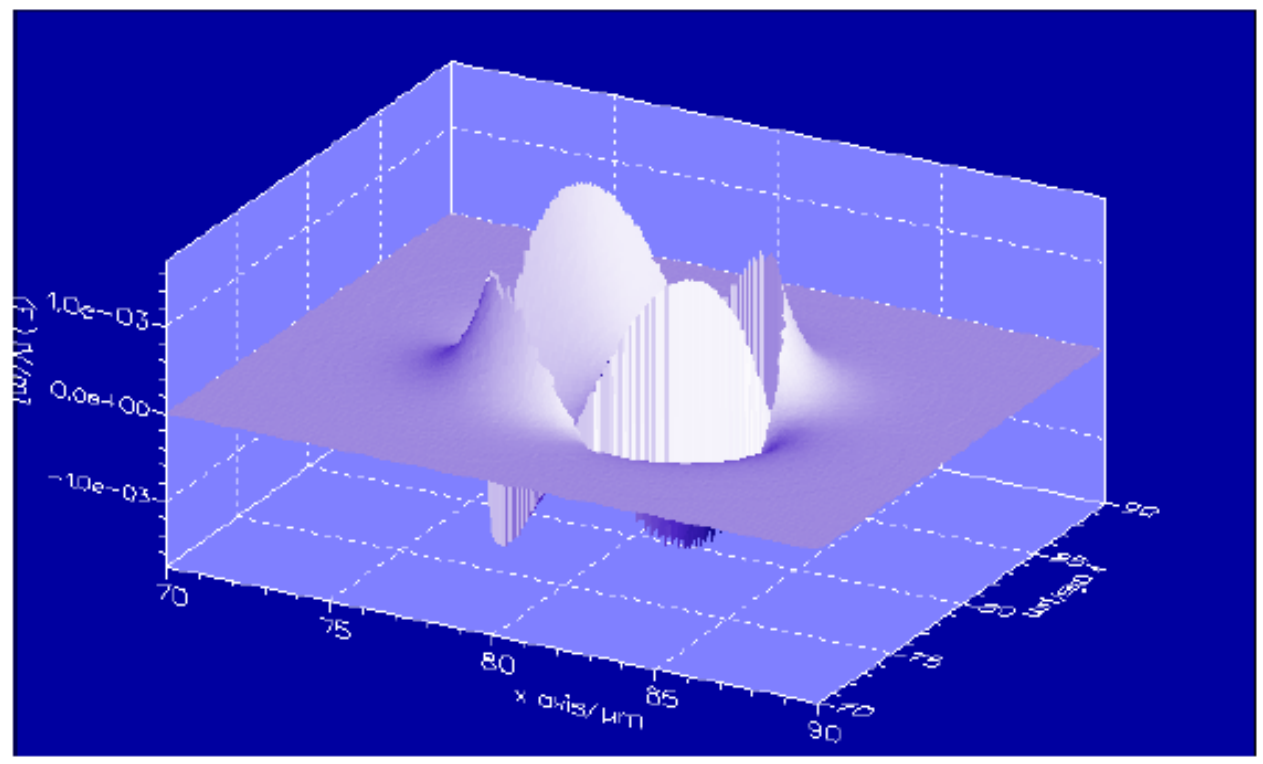


Appendix B: FIMMWAVE ${ }^{\circledR}$ Simulations Used For Spectral Width Validation

$1 \quad 1.462654$

21.462654

$3 \quad 1.461392$

$4 \quad 1.46139$

$5 \quad 1.46139$

$\begin{array}{ll}6 & 1.461388\end{array}$

$\begin{array}{ll}7 & 1.459772\end{array}$

$8 \quad 1.459772$

$9 \quad 1.459769$

$10 \quad 1.459769$

$\begin{array}{ll}11 & 1.459264\end{array}$

$12 \quad 1.459264$

$\begin{array}{ll}13 & 1.45787\end{array}$

$14 \quad 1.45787$

$\begin{array}{ll}15 & 1.457143\end{array}$

$\begin{array}{ll}16 & 1.457142\end{array}$

$\begin{array}{ll}17 & 1.457141\end{array}$

$\begin{array}{ll}18 & 1.457141\end{array}$

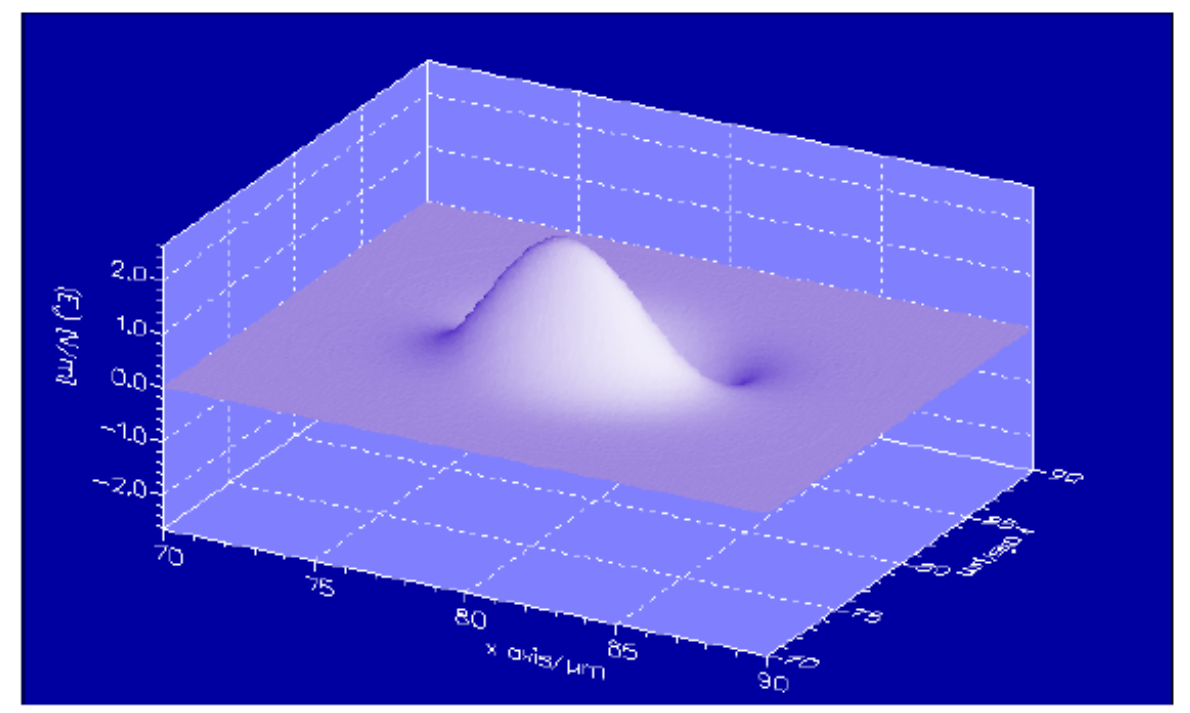

$1 \quad 1.462654$

$2 \quad 1.462654$

$3 \quad 1.461392$

$4 \quad 1.46139$

$5 \quad 1.46139$

$\begin{array}{ll}6 & 1.461388\end{array}$

$\begin{array}{ll}7 & 1.459772\end{array}$

$8 \quad 1.459772$

$9 \quad 1.459769$

$10 \quad 1.459769$

$\begin{array}{ll}11 & 1.459264\end{array}$

$12 \quad 1.459264$

$\begin{array}{ll}13 & 1.45787\end{array}$

$14 \quad 1.45787$

$\begin{array}{ll}15 & 1.457143\end{array}$

$\begin{array}{ll}16 & 1.457142\end{array}$

$17 \quad 1.457141$

$\begin{array}{ll}18 & 1.457141\end{array}$

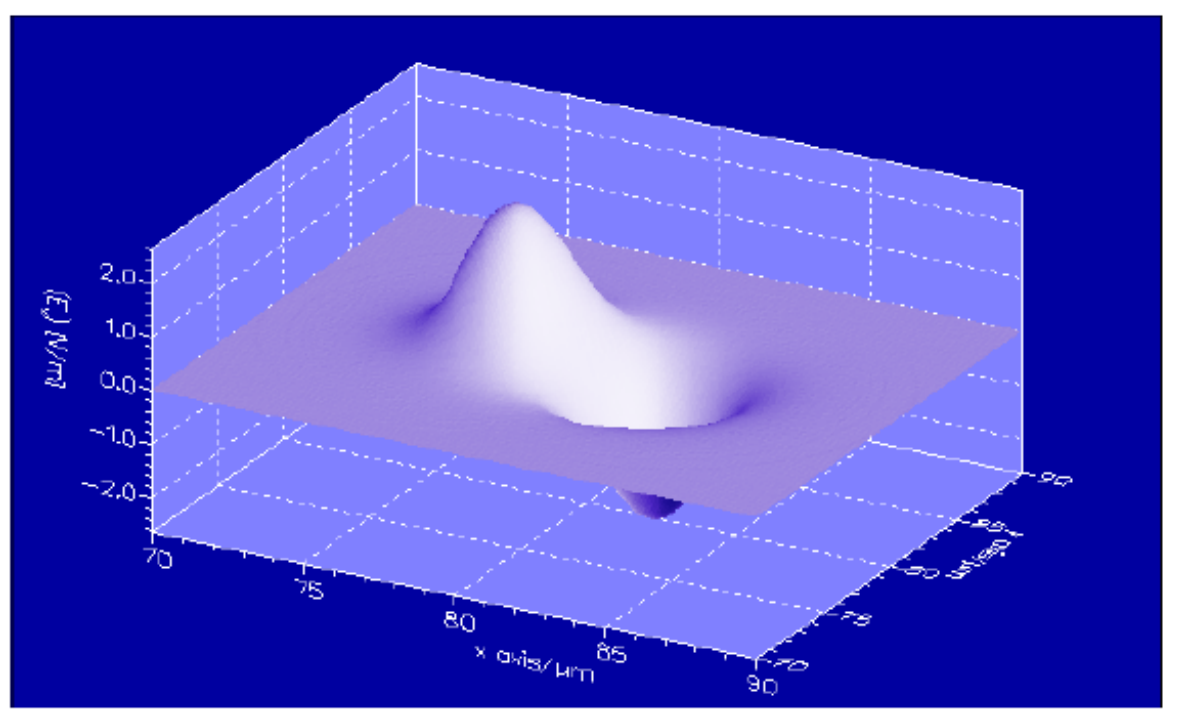




\section{Appendix B: FIMMWAVE ${ }^{\circledR}$ Simulations Used For Spectral Width Validation}

11.462654

21.462654

$3 \quad 1.461392$

$4 \quad 1.46139$

$5 \quad 1.46139$

$6 \quad 1.461388$

$7 \quad 1.459772$

$8 \quad 1.459772$

$9 \quad 1.459769$

101.459769

111.459264

121.459264

$\begin{array}{ll}13 & 1.45787\end{array}$

$\begin{array}{ll}14 & 1.45787\end{array}$

$\begin{array}{ll}15 & 1.457143\end{array}$

$\begin{array}{ll}16 & 1.457142\end{array}$

$\begin{array}{ll}17 & 1.457141\end{array}$

$\begin{array}{ll}18 & 1.457141\end{array}$

1.462654

21.462654

$\begin{array}{ll}3 & 1.461392\end{array}$

$4 \quad 1.46139$

$5 \quad 1.46139$

$\begin{array}{ll}6 & 1.461388\end{array}$

$\begin{array}{ll}7 & 1.459772\end{array}$

81.459772

$9 \quad 1.459769$

$\begin{array}{ll}10 & 1.459769\end{array}$

111.459264

121.459264

$\begin{array}{ll}13 & 1.45787\end{array}$

$14 \quad 1.45787$

$15 \quad 1.457143$

$\begin{array}{ll}16 & 1.457142\end{array}$

$17 \quad 1.457141$

$\begin{array}{ll}18 & 1.457141\end{array}$
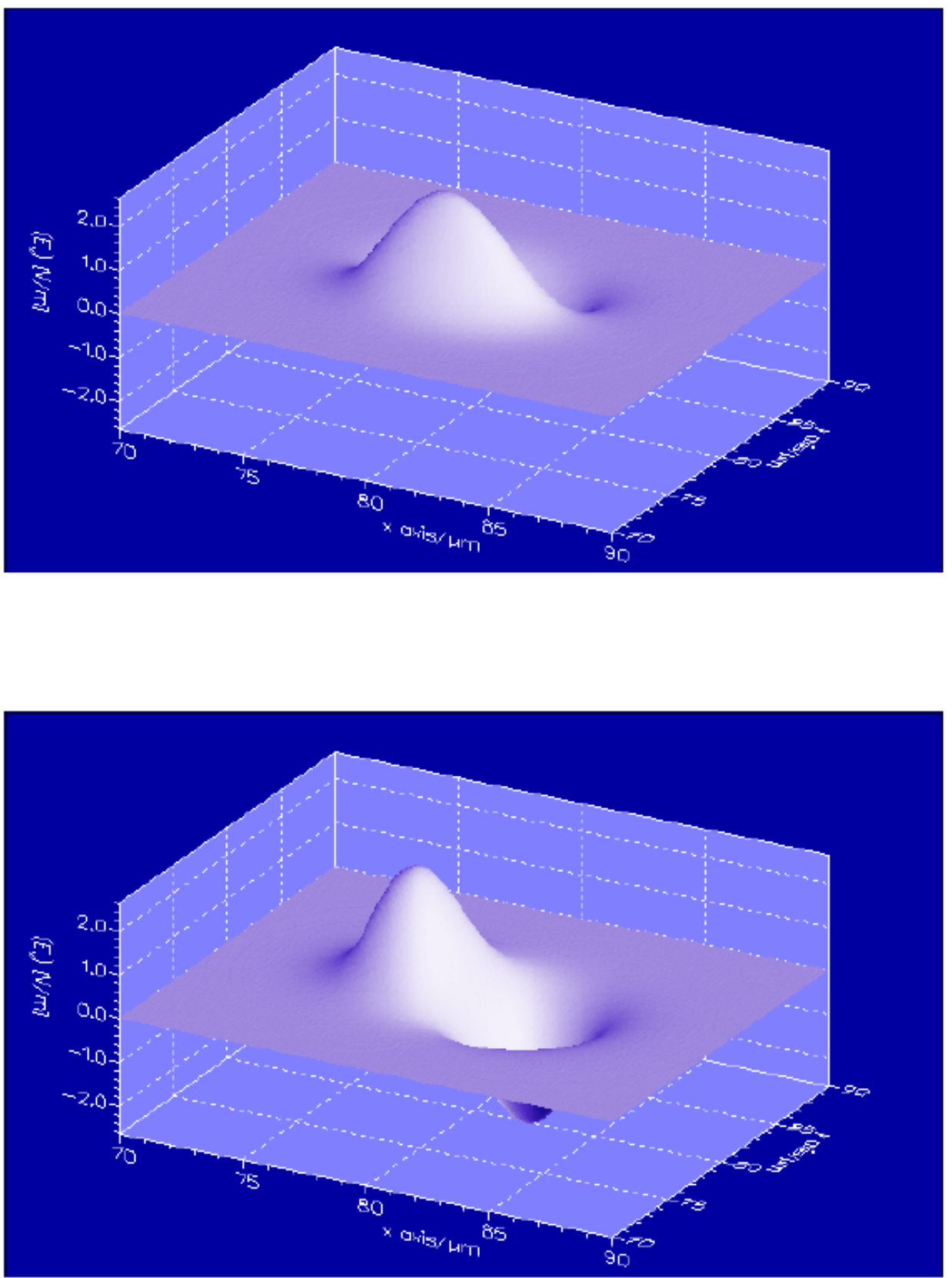


\section{Appendix B: FIMMWAVE ${ }^{\circledR}$ Simulations Used For Spectral Width Validation}

11.462654

21.462654

$3 \quad 1.461392$

$4 \quad 1.46139$

$5 \quad 1.46139$

$6 \quad 1.461388$

$7 \quad 1.459772$

$8 \quad 1.459772$

$9 \quad 1.459769$

$10 \quad 1.459769$

111.459264

$12 \quad 1.459264$

$13 \quad 1.45787$

$14 \quad 1.45787$

$\begin{array}{ll}15 & 1.457143\end{array}$

$16 \quad 1.457142$

$\begin{array}{ll}17 & 1.457141\end{array}$

$\begin{array}{ll}18 & 1.457141\end{array}$

$1 \quad 1.462654$

21.462654

$3 \quad 1.461392$

$4 \quad 1.46139$

$5 \quad 1.46139$

$\begin{array}{ll}6 & 1.461388\end{array}$

$\begin{array}{ll}7 & 1.459772\end{array}$

81.459772

9 1.459769

$10 \quad 1.459769$

111.459264

$\begin{array}{ll}12 & 1.459264\end{array}$

$13 \quad 1.45787$

$\begin{array}{ll}14 & 1.45787\end{array}$

$\begin{array}{ll}15 & 1.457143\end{array}$

$16 \quad 1.457142$

$\begin{array}{ll}17 & 1.457141\end{array}$

$18 \quad 1.457141$
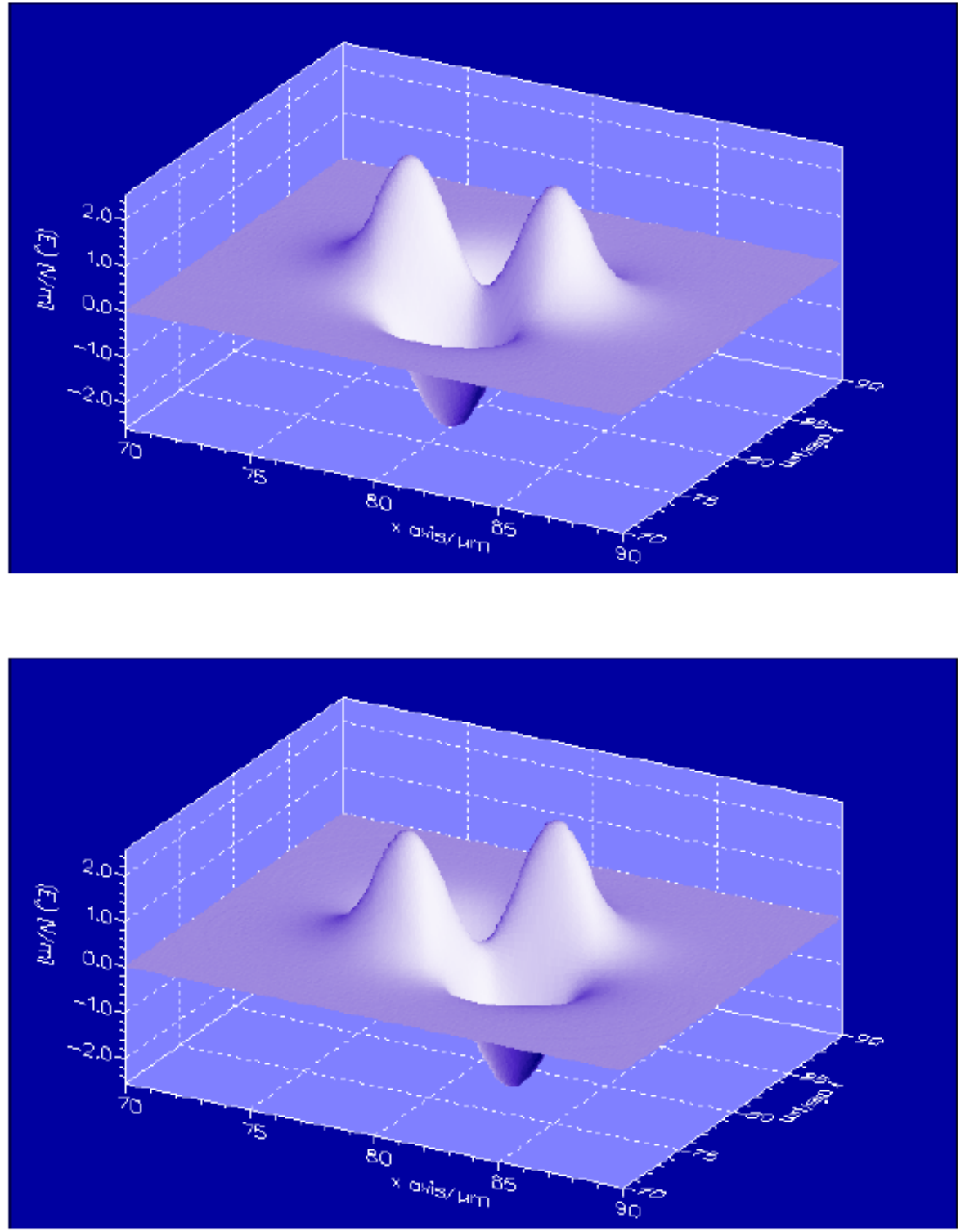
Appendix B: FIMMWAVE ${ }^{\circledR}$ Simulations Used For Spectral Width Validation
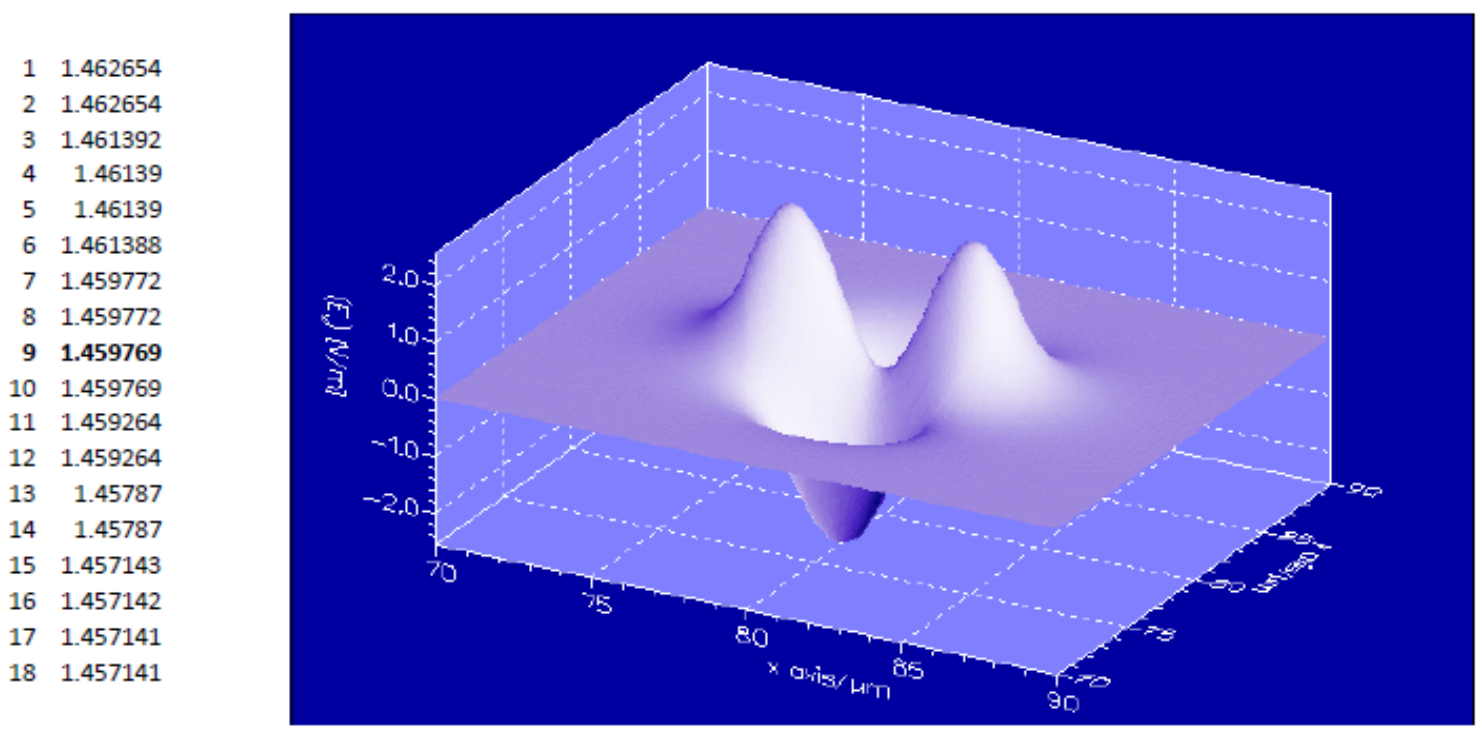

11.462654

$2 \quad 1.462654$

31.461392

$\begin{array}{ll}4 & 1.46139\end{array}$

$\begin{array}{ll}5 & 1.46139\end{array}$

$\begin{array}{ll}6 & 1.461388\end{array}$

$\begin{array}{ll}7 & 1.459772\end{array}$

81.459772

91.459769

$\begin{array}{ll}10 & 1.459769\end{array}$

$\begin{array}{ll}11 & 1.459264\end{array}$

$\begin{array}{ll}12 & 1.459264\end{array}$

$\begin{array}{ll}13 & 1.45787\end{array}$

$\begin{array}{ll}14 & 1.45787\end{array}$

$\begin{array}{ll}15 & 1.457143\end{array}$

$\begin{array}{ll}16 & 1.457142\end{array}$

$\begin{array}{ll}17 & 1.457141\end{array}$

$\begin{array}{ll}18 & 1.457141\end{array}$

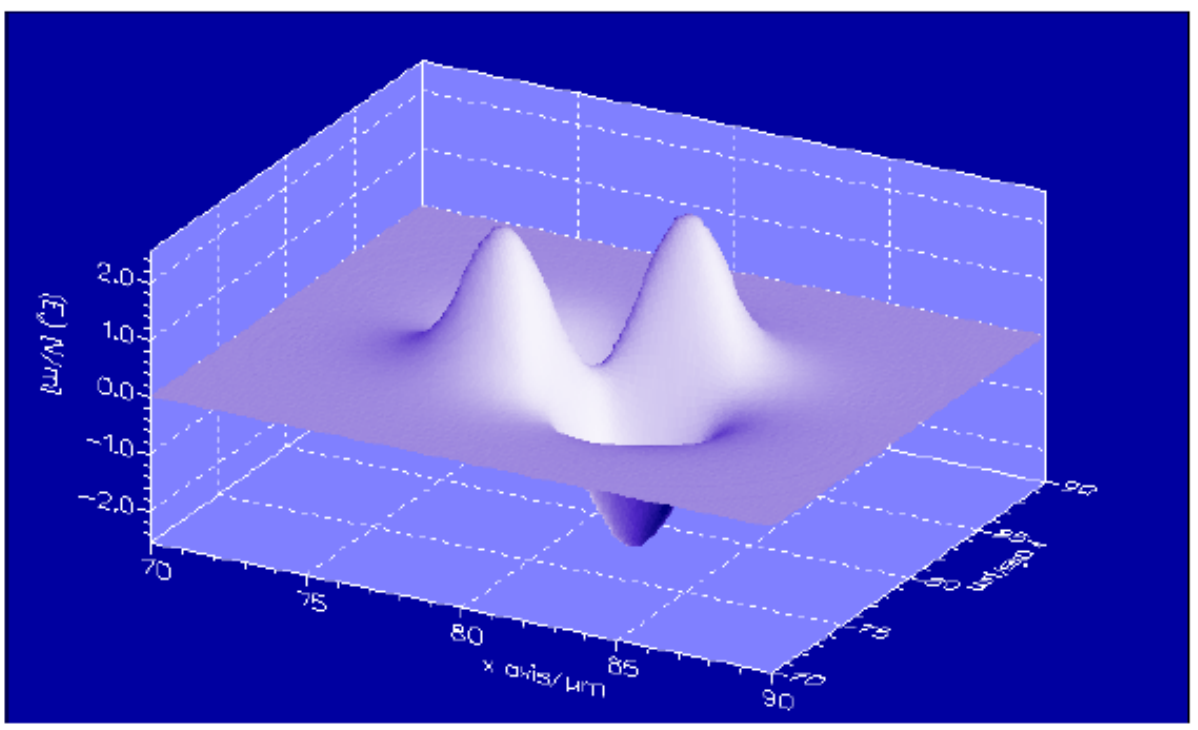




\section{Appendix B: FIMMWAVE ${ }^{\circledR}$ Simulations Used For Spectral Width Validation}

11.462654

21.462654

$3 \quad 1.461392$

$4 \quad 1.46139$

$5 \quad 1.46139$

$\begin{array}{ll}6 & 1.461388\end{array}$

$\begin{array}{ll}7 & 1.459772\end{array}$

$8 \quad 1.459772$

91.459769

$10 \quad 1.459769$

$11 \quad 1.459264$

$12 \quad 1.459264$

$13 \quad 1.45787$

$14 \quad 1.45787$

$15 \quad 1.457143$

$16 \quad 1.457142$

171.457141

$18 \quad 1.457141$

1.462654

$2 \quad 1.462654$

$\begin{array}{ll}3 & 1.461392\end{array}$

$4 \quad 1.46139$

$5 \quad 1.46139$

$6 \quad 1.461388$

$7 \quad 1.459772$

$8 \quad 1.459772$

$9 \quad 1.459769$

$10 \quad 1.459769$

$11 \quad 1.459264$

$12 \quad 1.459264$

$13 \quad 1.45787$

$14 \quad 1.45787$

$\begin{array}{ll}15 & 1.457143\end{array}$

$16 \quad 1.457142$

$\begin{array}{ll}17 & 1.457141\end{array}$

$18 \quad 1.457141$
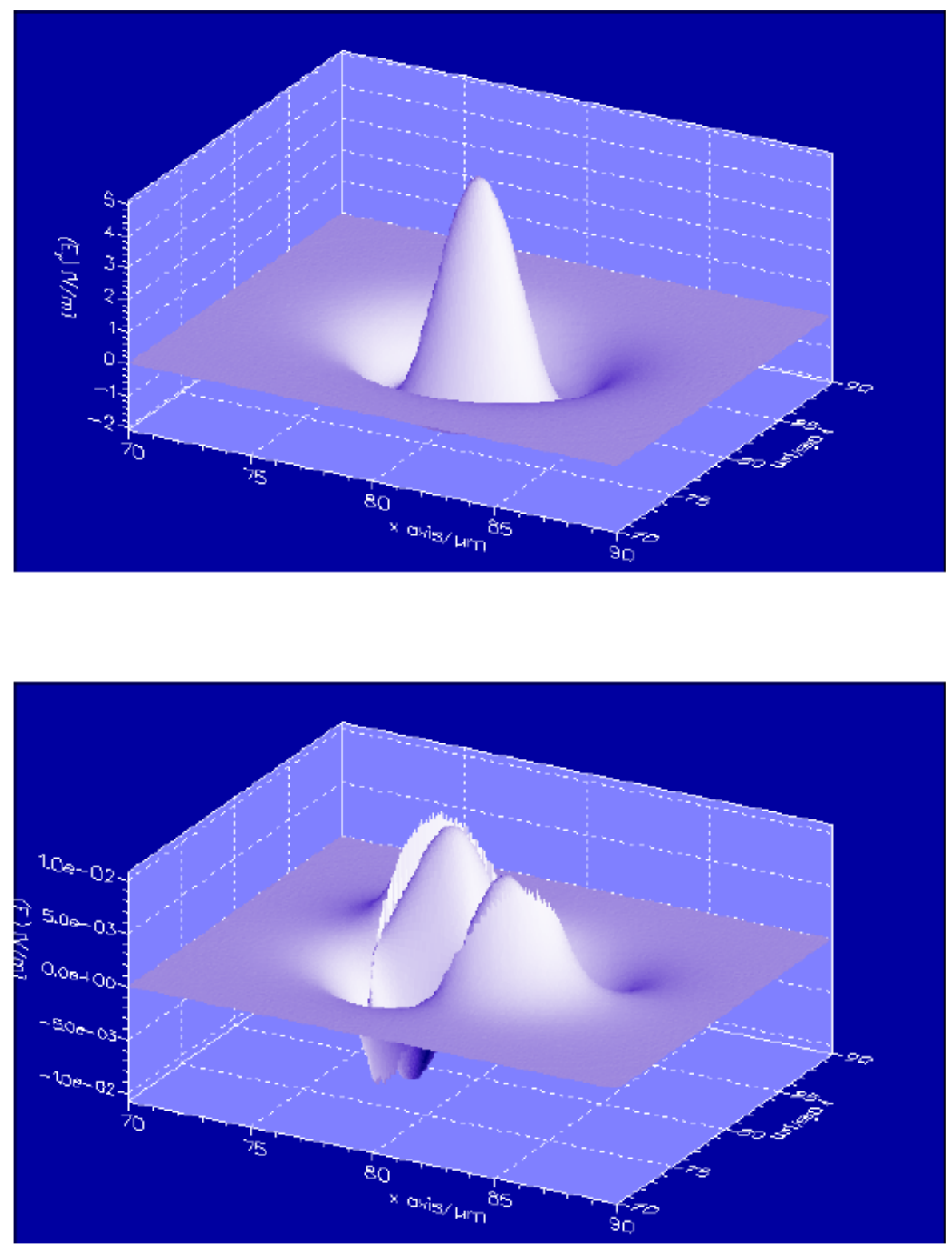
Appendix B: FIMMWAVE ${ }^{\circledR}$ Simulations Used For Spectral Width Validation

$1 \quad 1.462654$

$2 \quad 1.462654$

$3 \quad 1.461392$

$4 \quad 1.46139$

$5 \quad 1.46139$

$6 \quad 1.461388$

$\begin{array}{ll}7 & 1.459772\end{array}$

$8 \quad 1.459772$

$\begin{array}{ll}9 & 1.459769\end{array}$

101.459769

$11 \quad 1.459264$

$12 \quad 1.459264$

$13 \quad 1.45787$

$14 \quad 1.45787$

$15 \quad 1.457143$

$16 \quad 1.457142$

$\begin{array}{ll}17 & 1.457141\end{array}$

$18 \quad 1.457141$

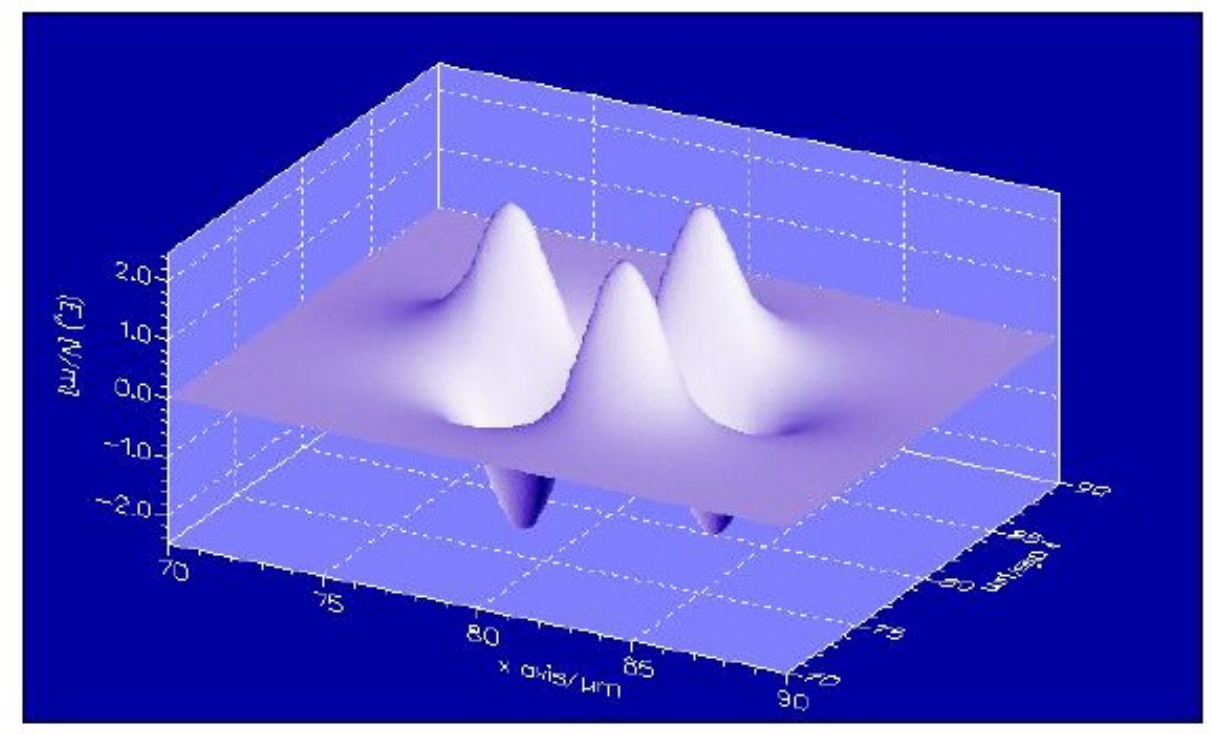

11.462654

21.462654

$3 \quad 1.461392$

$4 \quad 1.46139$

$5 \quad 1.46139$

$6 \quad 1.461388$

$\begin{array}{ll}7 & 1.459772\end{array}$

$8 \quad 1.459772$

$9 \quad 1.459769$

$10 \quad 1.459769$

111.459264

121.459264

$\begin{array}{ll}13 & 1.45787\end{array}$

$14 \quad 1.45787$

$15 \quad 1.457143$

$\begin{array}{ll}16 & 1.457142\end{array}$

$17 \quad 1.457141$

$18 \quad 1.457141$

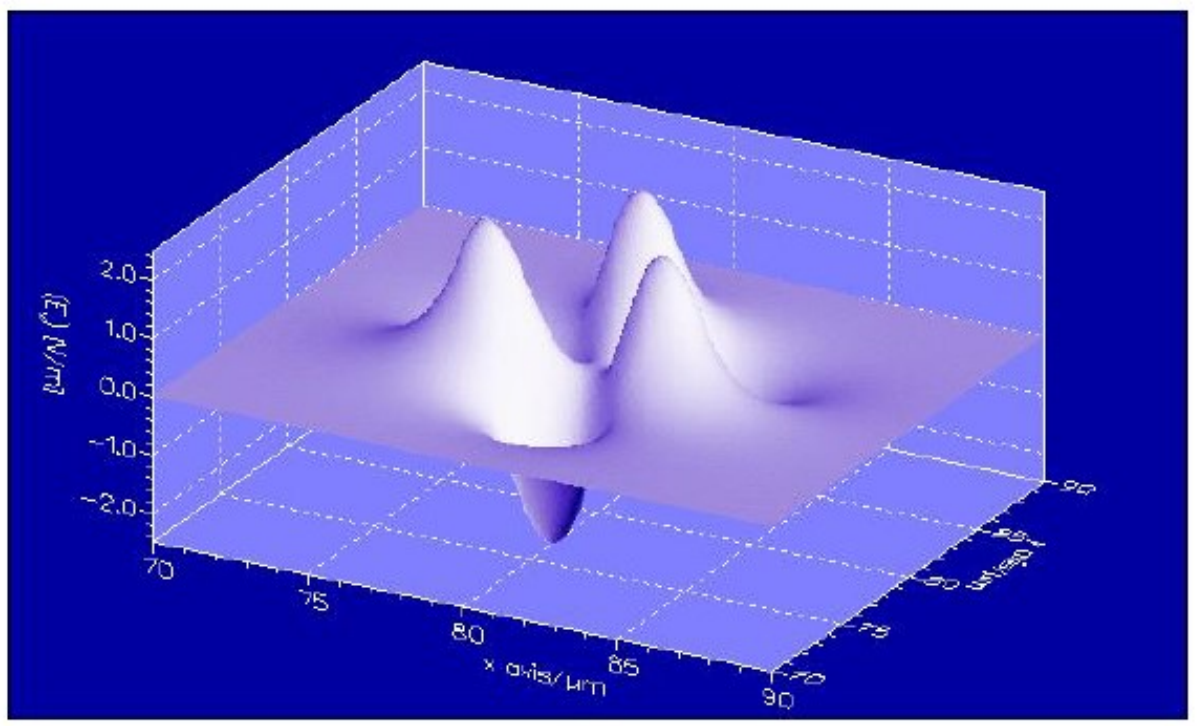




\section{Appendix B: FIMMWAVE ${ }^{\circledast}$ Simulations Used For Spectral Width Validation}

11.462654

21.462654

$3 \quad 1.461392$

$4 \quad 1.46139$

$5 \quad 1.46139$

$6 \quad 1.461388$

$7 \quad 1.459772$

81.459772

$9 \quad 1.459769$

$10 \quad 1.459769$

$11 \quad 1.459264$

$12 \quad 1.459264$

$13 \quad 1.45787$

$14 \quad 1.45787$

$15 \quad 1.457143$

$16 \quad 1.457142$

$17 \quad 1.457141$

$18 \quad 1.457141$

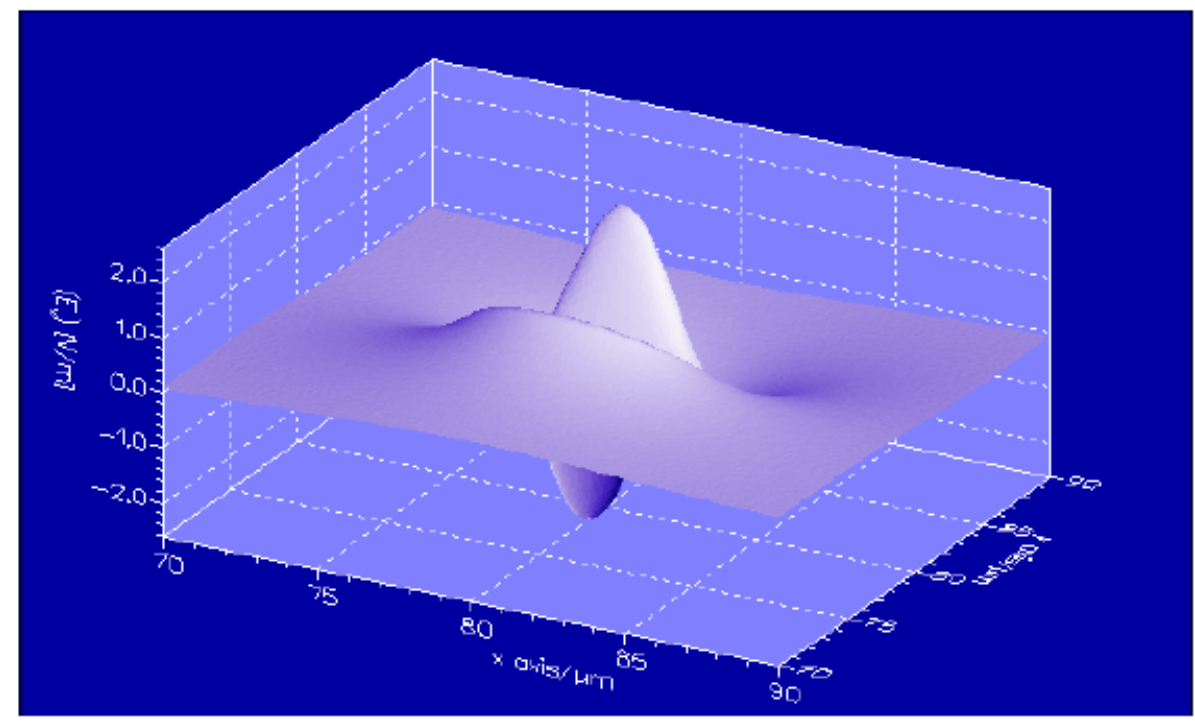

11.462654

21.462654

31.461392

$4 \quad 1.46139$

1.46139

$6 \quad 1.461388$

$\begin{array}{ll}7 & 1.459772\end{array}$

$8 \quad 1.459772$

91.459769

$10 \quad 1.459769$

$11 \quad 1.459264$

121.459264

$13 \quad 1.45787$

$14 \quad 1.45787$

$15 \quad 1.457143$

$16 \quad 1.457142$

$17 \quad 1.457141$

$18 \quad 1.457141$

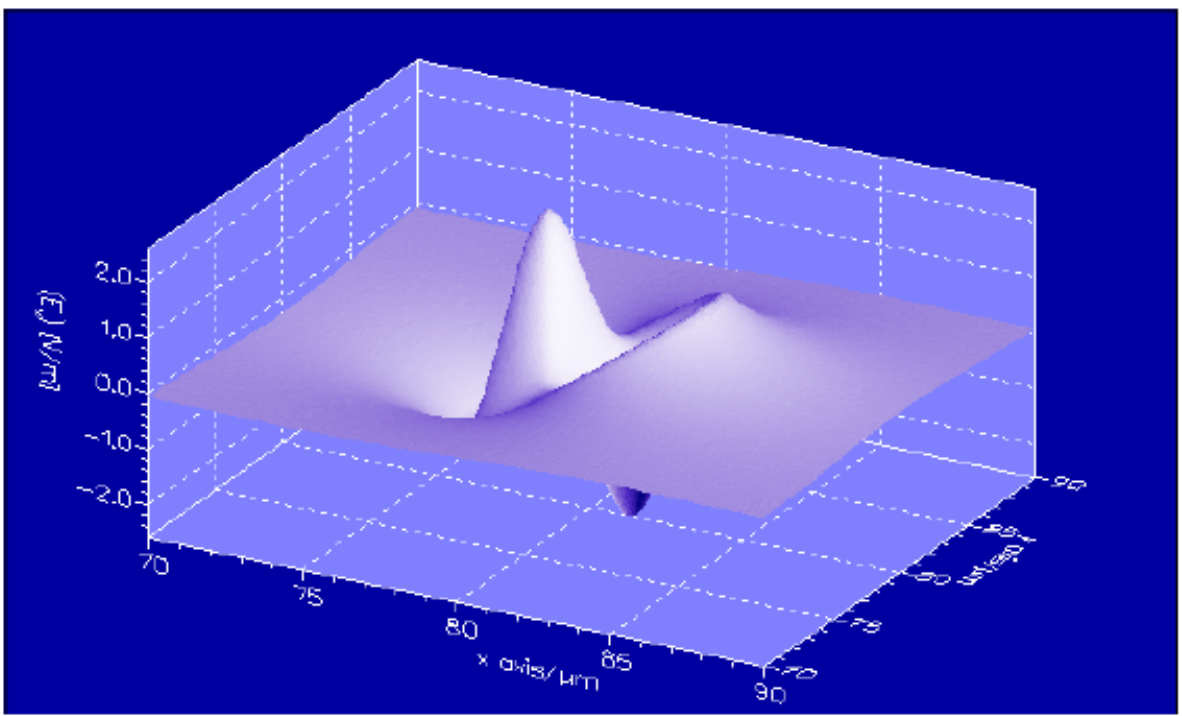




\section{Appendix B: FIMMWAVE ${ }^{\circledR}$ Simulations Used For Spectral Width Validation}

$1 \quad 1.462654$

21.462654

$\begin{array}{ll}3 & 1.461392\end{array}$

$4 \quad 1.46139$

$5 \quad 1.46139$

$6 \quad 1.461388$

$\begin{array}{ll}7 & 1.459772\end{array}$

$8 \quad 1.459772$

$9 \quad 1.459769$

$10 \quad 1.459769$

111.459264

121.459264

$13 \quad 1.45787$

$14 \quad 1.45787$

151.457143

$16 \quad 1.457142$

$17 \quad 1.457141$

$18 \quad 1.457141$

11.462654

21.462654

$3 \quad 1.461392$

$4 \quad 1.46139$

$5 \quad 1.46139$

$\begin{array}{ll}6 & 1.461388\end{array}$

$\begin{array}{ll}7 & 1.459772\end{array}$

$8 \quad 1.459772$

$9 \quad 1.459769$

$10 \quad 1.459769$

$11 \quad 1.459264$

$12 \quad 1.459264$

$13 \quad 1.45787$

$14 \quad 1.45787$

$15 \quad 1.457143$

$16 \quad 1.457142$

$17 \quad 1.457141$

$18 \quad 1.457141$
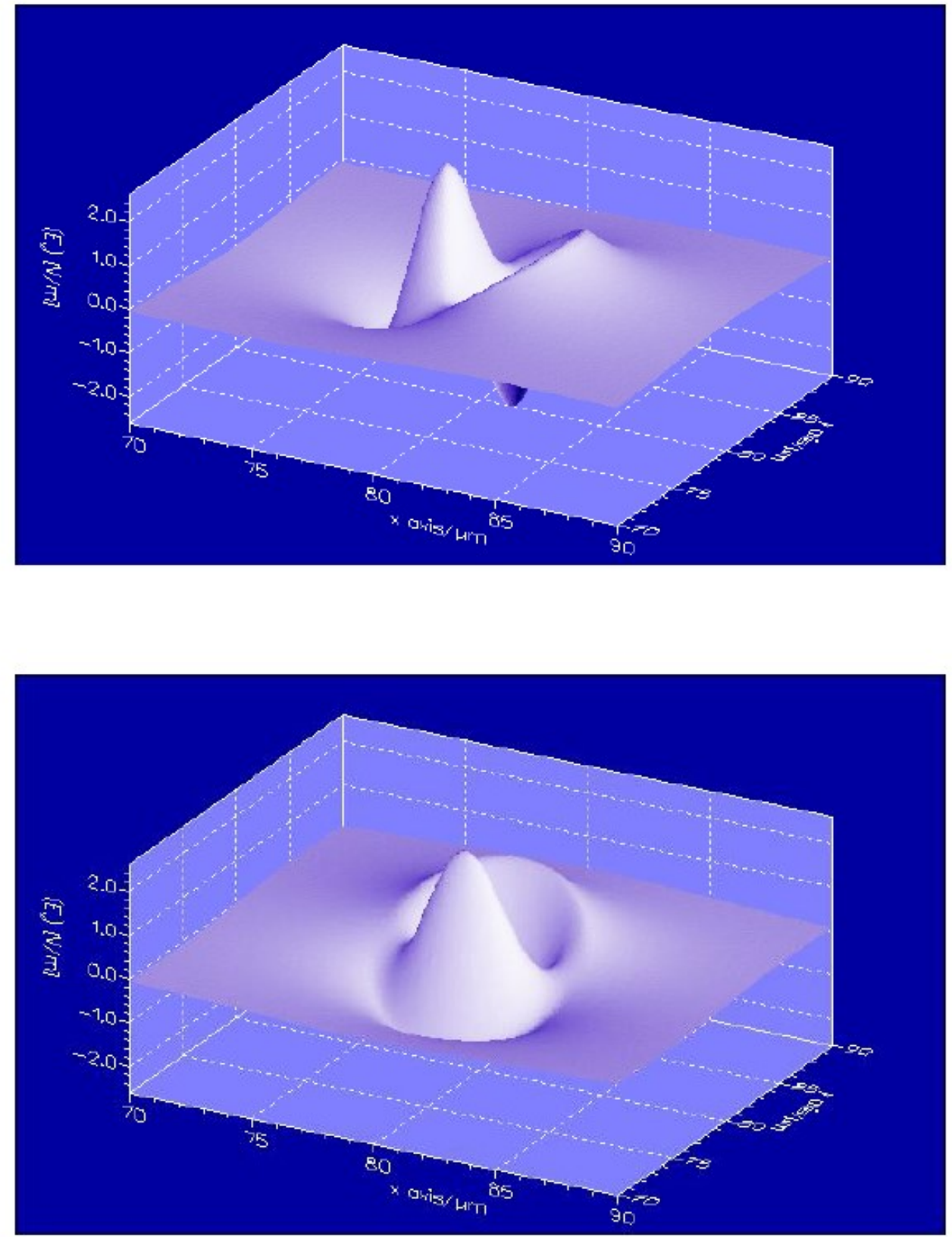


\section{Appendix C: Derivation of Coupled Mode Analysis}

We begin by assuming an input mode $\left(E_{1}, H_{1}\right)$ entering a waveguide $\left(E_{2}, H_{2}\right)$

$$
\begin{array}{cc}
\vec{V} \times \vec{E}_{1}=-j \omega \mu \vec{H}_{1} & \vec{V} \times \vec{E}_{2}=-j \omega \mu \vec{H}_{2} \\
\vec{V} \times \vec{H}_{1}=-j \omega \mu \vec{E}_{1}+\vec{P}_{1} & \vec{V} \times \vec{H}_{2}=-j \omega \mu \vec{E}_{2}
\end{array}
$$

$\left(\vec{E}_{2}, \vec{H}_{2}\right)$ is a mode of the waveguide $\rightarrow$ i.e. one of the eigenmodes of the problem.

$\left(\vec{E}_{1}, \vec{H}_{1}\right)$ is a field generated by $\vec{P}_{1}$.

$$
\begin{gathered}
\nabla\left(\vec{E}_{1} \times \vec{H}_{2}+\vec{E}_{2} \times \vec{H}_{1}\right)=-j \omega \vec{P}_{1} \cdot \vec{E}_{2} \\
\iint_{\infty} d x d y \frac{\partial}{\partial z}\left[\vec{E}_{1} \times \vec{H}_{2}+\vec{E}_{2} \times \vec{H}_{1}\right\rfloor_{z}=-j \omega \iint_{\infty} d x d y \vec{P}_{1} \cdot \vec{E}_{2}
\end{gathered}
$$

The eigenmodes are given by $\left(\vec{e}_{n}, n_{n}\right)$ pairs of transverse fields (neglecting $\hat{z}$ fields). The backward propagating eigenmode is identical to the forward one except that $\vec{h}_{n}=-\vec{h}_{n}$ in order to have $\vec{e}_{n} \times n_{n}= \pm \hat{z}$.

If we choose $\left(\vec{E}_{2}, \vec{H}_{2}\right)$ as a backward propagating mode n:

$$
\left(\vec{E}_{2}\right)_{t}=\vec{e}_{n} \exp \left(+J \beta_{n} z\right) \quad\left(\vec{H}_{2}\right)_{t}=-h_{n} \exp \left(+j \beta_{n} z\right)
$$

For field 1, the most general solution (of the Helmholtz equation) is:

$$
\begin{aligned}
& \vec{E}_{1}^{\prime}=\sum_{m} a_{m} \vec{e}_{m} \exp \left(-J \rho_{m} z\right)+v_{m} \vec{e}_{m} \exp \left(+J \rho_{m} z\right) \\
& \vec{H}_{1}=\sum_{m} a_{m} \vec{h}_{m} \exp \left(-j \beta_{m} z\right)-b_{m} \vec{h}_{m} \exp \left(+j \beta_{m} z\right)
\end{aligned}
$$

$\vec{e}$ and $\vec{h}$ have the same amplitude factors because they are solved simultaneously.

If we replace into LHS of (4): 


\section{Appendix C: Derivation of Coupled Mode Analysis}

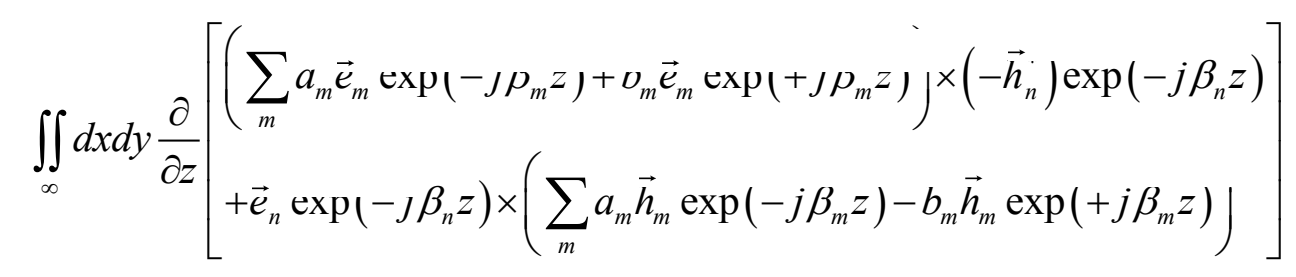

Note $\vec{h}_{m}=\vec{h}_{m}$ and $\vec{e}_{m}=\vec{e}_{m}$ and (C.8) $=0$ if $n \neq m$.

$$
\begin{aligned}
& \iint_{\infty} d x d y \frac{\partial}{\partial z}\left[\begin{array}{lll}
-a_{m}\left(\vec{e}_{m} \times n_{n}\right)^{e} & \cdots & -v_{m}\left(\vec{e}_{m} \times n_{n}\right)^{e} \\
+a_{m}\left(\vec{e}_{n} \times n_{m}\right)^{e} \cdots \cdots & -v_{m}\left(\vec{e}_{n} \times n_{m}\right)^{e \cdots \cdots}
\end{array}\right] \\
& \iint_{\infty} d x d y \frac{\partial}{\partial z}\left[-2 b_{m}\left(\vec{e}_{m} \times n_{n}\right)^{\left.e^{\jmath N_{n}} \beta_{m}\right) z}\right]
\end{aligned}
$$

Using RHS, changing syntax for clarifying integrals and rearranging we obtain:

$$
\frac{\partial}{\partial z}\left(-2 b_{m} e^{-j\left(\beta_{n}-\beta_{m}\right) z}\right) \iint_{\infty}\left(\vec{e}_{m} \times n_{n}\right) a x a y=-j \omega \int_{\infty} \Gamma_{1} \cdot \vec{e}_{n} e^{\cdots \cdots} \text { axay }
$$

Using $m=n$ we further arrange (9) and obtain:

$$
b_{m}^{\prime}=\frac{j \omega}{2} \frac{\iint_{\infty} \vec{P}_{1} \cdot \vec{e}_{m} e^{\cdots \cdots} a x d y}{\iint_{\infty}\left(\vec{e}_{m} \times n_{m}\right) u x d y}
$$

Where $b_{m}^{\prime}=\frac{\partial}{\partial z} b_{m}$ and similarly for $a_{m}$ :

$$
a_{m}^{\prime}=-\frac{j \omega}{2} \frac{\iint_{\infty} \vec{P}_{1} \cdot \vec{e}_{m} e^{\cdots \cdots} a x d y}{\iint_{\infty}\left(\vec{e}_{m} \times n_{m}\right) a x d y}
$$

We note that for some derivations, the denominator (including the factor of 2 ) is arbitrarily set to 4 . Note also that in some texts $a_{\mu}$ and $b_{\mu}$ are used instead of $A_{\mu}$ and $B_{\mu}$.

Using the same eigenfunctions to write down $\vec{P}$ : 


\section{Appendix C: Derivation of Coupled Mode Analysis}

$$
\vec{P}_{t}=\Delta \varepsilon E_{t}=\Delta \varepsilon \sum_{v}\left(a_{v}+b_{v}\right) E_{t v}
$$

In the case of a single mode excitation (forward, with amplitude $a_{0}$ ):

$$
\vec{P}_{t}=\Delta \varepsilon \vec{e}_{0 t} e^{\cdots}=a_{0} \varepsilon_{o} \vec{e}_{0 t} \Delta n t e^{\cdots=}=a_{0} \varepsilon_{o} \Delta n \Delta \Delta r \vec{e}_{0 t} e^{\cdots}
$$

Note this is valid for $\mathrm{x}$ or $\mathrm{y}$ polarized excitation.

Replace into (C.12) and (C.13):

$$
\begin{aligned}
& b_{m}^{\prime}=\frac{j \omega \varepsilon_{0} \not \chi_{n}}{\not 2} \frac{\iint_{\infty} a_{0} \vec{e}_{0} \cdot \vec{e}_{m} e^{\left.3_{m}\right) z} \Delta n d x d y}{\iint_{\infty}\left(\vec{e}_{m} \times n_{m}\right)^{u x d y}} \\
& a_{m}^{\prime}=\frac{-j \omega \varepsilon_{0} \not \chi_{n}}{\not 2} \frac{\iint_{\infty} a_{0} \vec{e}_{0} \cdot \vec{e}_{m} e^{\left.{ }^{3}\right) z} \Delta n d x d y}{\iint_{\infty}\left(\vec{e}_{m} \times n_{m}\right) a x d y}
\end{aligned}
$$

Cleaning up, we obtain for the change in amplitude of the backward going mode $b_{m}^{\prime}$ :

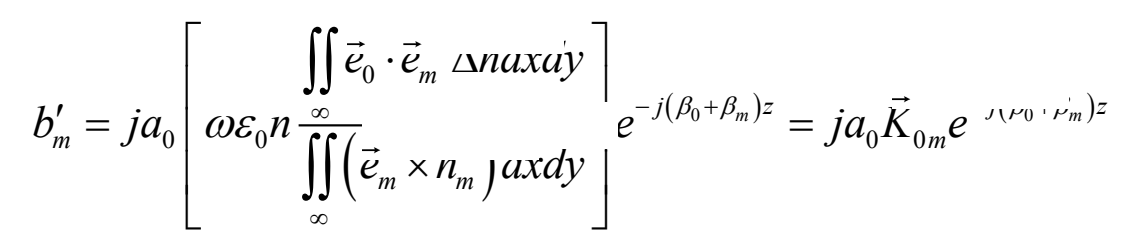

Note $\beta_{0}$ is positive for backward mode and $\beta_{m}$ is positive for forward mode. The change in amplitude of the $a_{0}$ mode is given by (due to $b_{m} e^{j \beta_{m} z} \vec{e}_{m}$ ):

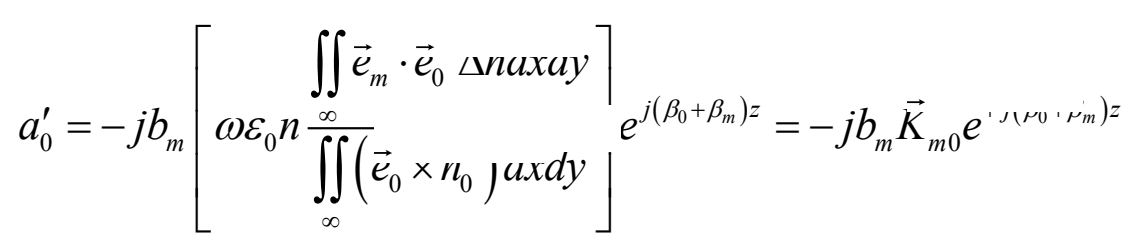

With positive $\beta_{0}$ and $\beta_{m}$, and where, for both (C.18) and (C.19): 


\section{Appendix C: Derivation of Coupled Mode Analysis}

$$
\vec{K}_{m n}=\omega \varepsilon_{0} n \frac{\iint_{\infty} \vec{e}_{m} \cdot \vec{e}_{n} \Delta \text { rlaxuy }}{\iint_{\infty}\left(\vec{e}_{n} \times n_{n}\right) a x d y}
$$

For sinusoidal perturbations:

$$
\Delta n=\Delta n_{0} \cos \frac{2 \pi z}{\Lambda}=\Delta n_{0} \cos \mathrm{K} z=\Delta n_{0}\left(\frac{e^{i \mathrm{~K} z}+e^{-i \mathrm{~K} z}}{2}\right)
$$

Substituting into (C.19) and (C.18), respectively, while noting that positive $\mathrm{K}$ term integrates to zero when used in (C.21) and vice-versa for (C.19), we obtain:

$$
\begin{aligned}
& a_{0}^{\prime}=-j b_{m}\left[\omega \varepsilon_{0} n \frac{\iint_{\infty} \vec{e}_{m} \cdot \vec{e}_{0} \Delta n_{0} a x d y}{\iint_{\infty}\left(\vec{e}_{0} \times n_{0}\right) a x d y} \frac{e^{j\left(\beta_{0}+\beta_{m}-\mathrm{K}\right) z}}{2}\right. \\
& b_{m}^{\prime}=+j a_{0}\left[\omega \varepsilon_{0} n \frac{\left.\iint_{\infty}^{\infty} \vec{e}_{0} \cdot \vec{e}_{m} \Delta r_{0} a x d y\right]}{\iint_{\infty}\left(\vec{e}_{m} \times n_{m}\right) a x d y} \frac{e^{-j\left(\beta_{0}+\beta_{m}-\mathrm{K}\right) z}}{2}\right.
\end{aligned}
$$

Here we have as the phase-matching condition $2 \delta=\beta_{0}+\beta_{m}-\mathrm{K} \cong 0$, and with $m n=m 0$ or $0 m$ :

$$
K_{m n}=\frac{\omega \varepsilon_{0} n}{2} \frac{\iint_{\infty} \vec{e}_{m} \cdot \vec{e}_{n} \Delta r_{0} u x d y}{\iint_{\infty}\left(\vec{e}_{n} \times n_{n}\right) u x d y}
$$

For the Bragg mode (C.24) reduces to:

$$
K_{m m}=\frac{\omega \varepsilon_{0} n}{2} \Delta n_{0} Z_{0} \frac{\iint_{\infty} \vec{e}_{m} \cdot r_{m} a x a y}{\iint_{\infty}\left(\vec{e}_{m} \times n_{m}\right) a x d y}
$$




\section{Appendix C: Derivation of Coupled Mode Analysis}

Which further reduces to the well-known Bragg condition:

$$
K_{m m}=\frac{\not 2 \pi f \varepsilon_{0} \not \Delta \Delta n_{0}}{\not 2} \sqrt{\frac{\mu_{0}}{\varepsilon_{0}}} \frac{1}{\not h} \eta=\pi f \sqrt{\varepsilon_{0} \mu_{0}} \Delta n_{0} \eta=\frac{\pi \Delta n_{0}}{\lambda_{0}} \eta
$$

So Bragg mode and backward going mode coupling is summarized through:

$$
\begin{aligned}
& a_{0}^{\prime}=-j b_{m} K_{m 0} e^{j 2 \delta z} \\
& b_{m}^{\prime}=+j a_{0} K_{0 m} e^{-j 2 \delta z} \\
& A(z)=a_{0}(z) e^{-j \delta z} \\
& B(z)=b_{m}(z) e^{j \delta z}
\end{aligned}
$$

Define:

Relating this set of equations (C.27-30) we find:

$$
\begin{aligned}
& \frac{d A}{d z}+j \delta A=-j K_{m 0} B \\
& \frac{d B}{d z}-j \delta B=+j K_{0 m} A
\end{aligned}
$$

Standard solution from above only works if $K_{0 m}=K_{m 0}$, which is true only if $\iint_{\infty} \vec{e}_{m} \times n_{m}=\mathrm{Jj} \vec{e}_{0} \times n_{0}$. 


\section{Appendix D: Statistical Comparison of Empirical/Theoretical Values}

In order to confirm the statistical significance we use a Chi Squared Distribution

$$
\chi^{2}=\frac{1}{N-1} \sum_{m, \lambda} \frac{\left(\alpha_{m, \lambda-\text { theory }}-\alpha_{m, \lambda \text {-experimental }}\right)^{2}}{\alpha_{m, \lambda-\text { theory }}}
$$

where for the data in Figure 4.2.1 we get a chi squared value of

$$
\chi^{2}=0.00444
$$

This value of chi squared implies a statistical significance at the $95^{\text {th }}$ percentile.

Yet another validation is the sample variance calculated using

$$
s^{2}=\frac{1}{N-1} \sum_{m, \lambda}\left(\alpha_{m, \lambda-\text { theory }}-\alpha_{m, \lambda \text {-experimental }}\right)^{2}
$$

to get a variance of

$$
s^{2}=0.1
$$

The square root of this value is $0.3^{\circ}$. This value is called the "root mean square", or RMS, and is in this case an average measure of variation between the experimental and expected values. We note that the RMS value is larger than the reading error of the angle measurements. In summary, the observed results show a near-certain agreement with expected values. 
Appendix E: Diagrams of Visible Diffraction Orders for 1550nm TFBG

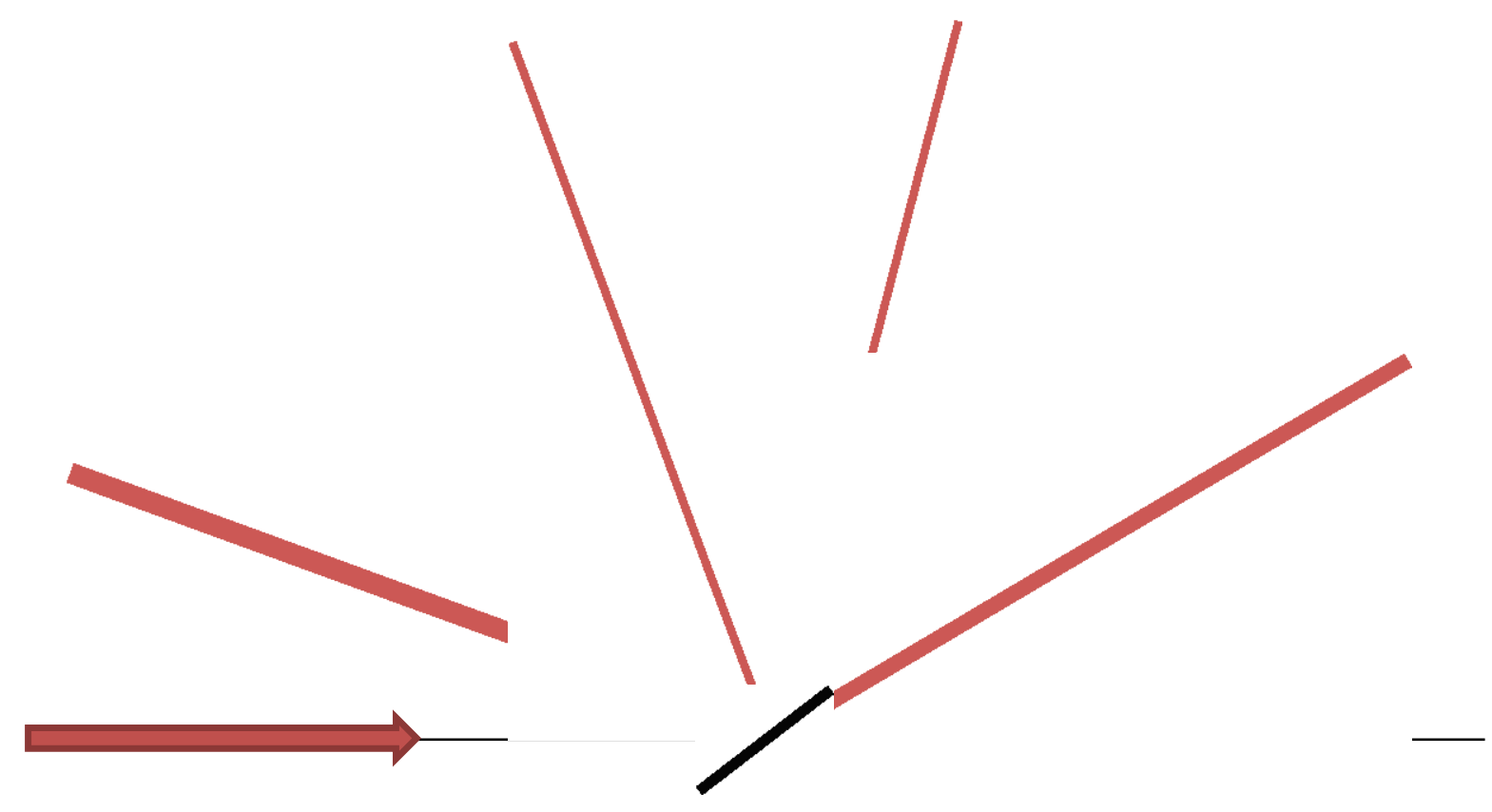

$647.1 \mathrm{~nm}$

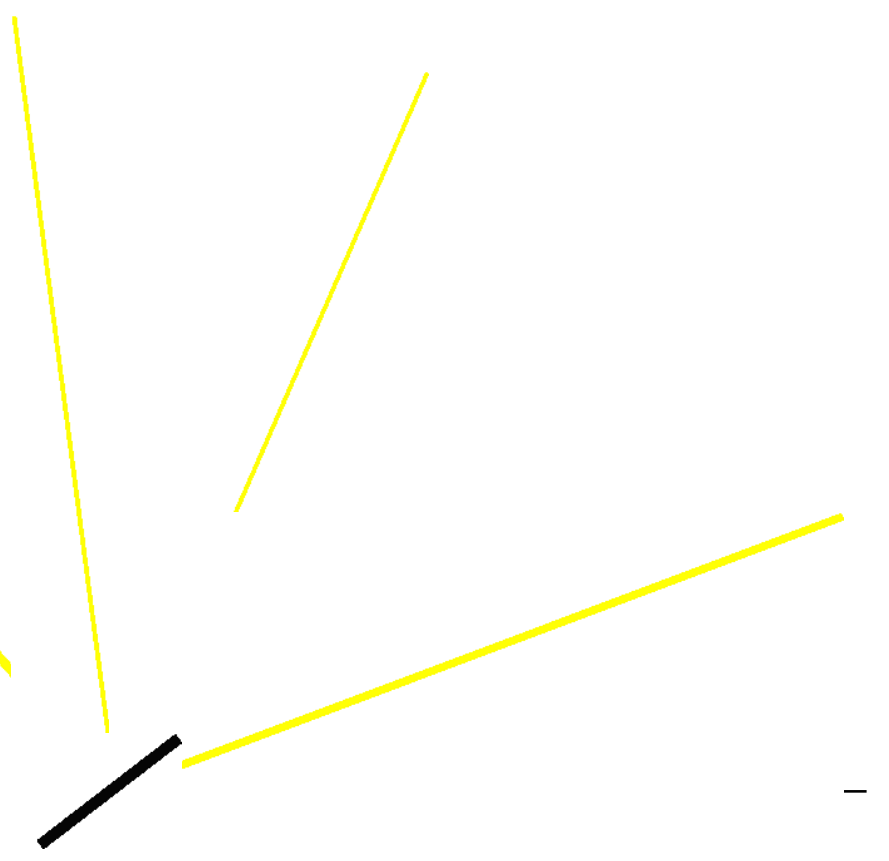

$568.2 \mathrm{~nm}$ 
Appendix E: Diagrams of Visible Diffraction Orders for 1550nm TFBG

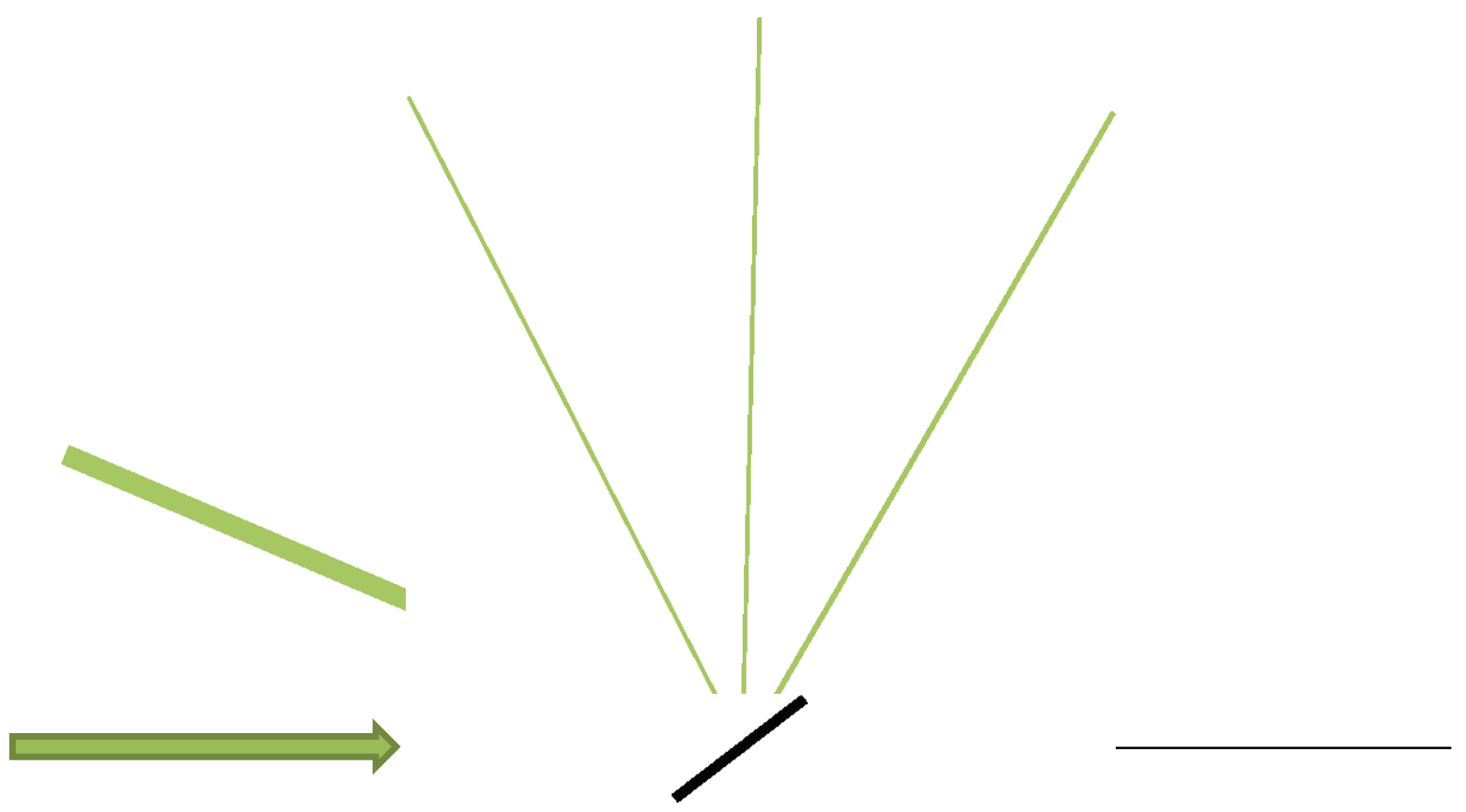

$514.5 \mathrm{~nm}$

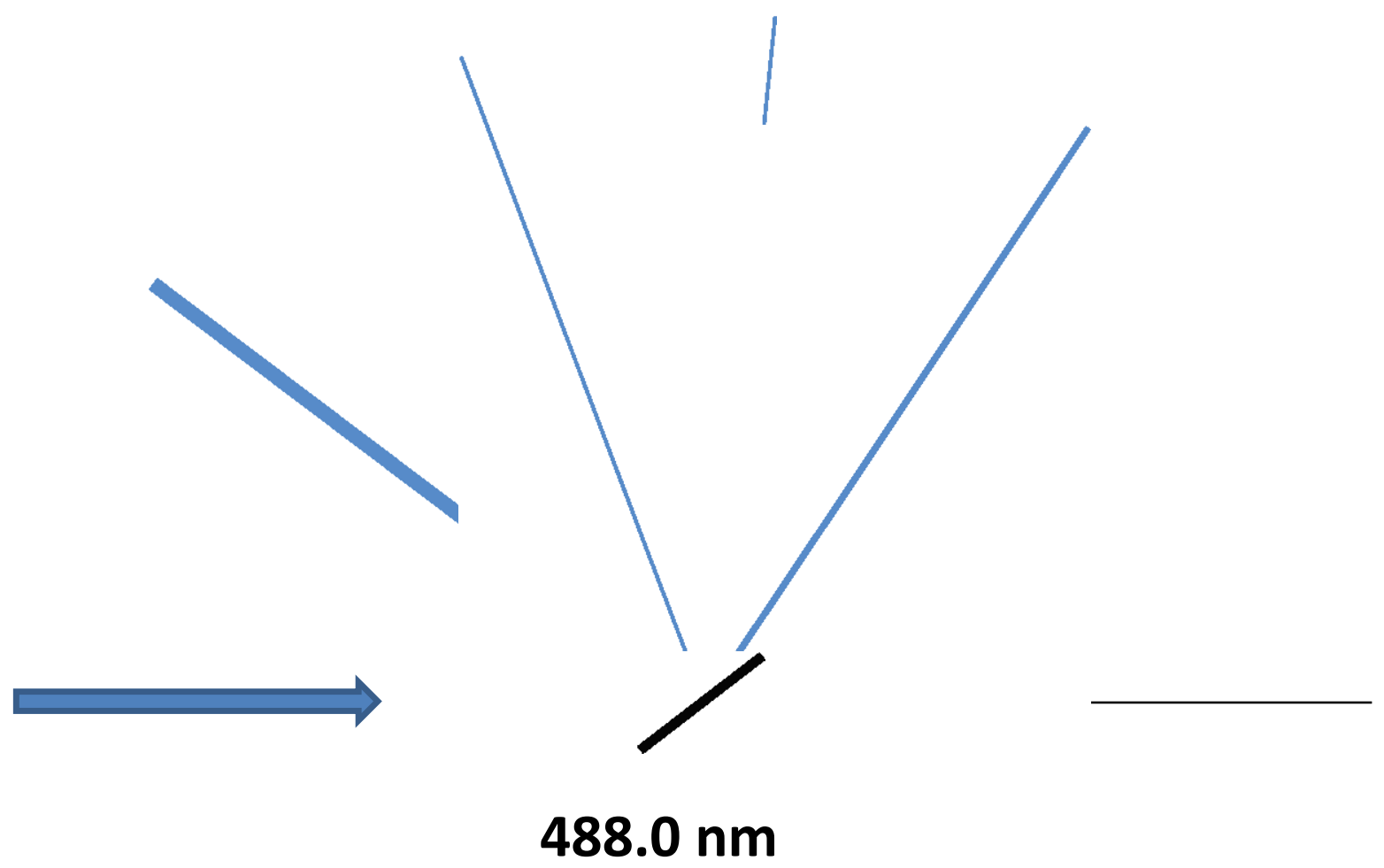


Appendix E: Diagrams of Visible Diffraction Orders for 1550nm TFBG

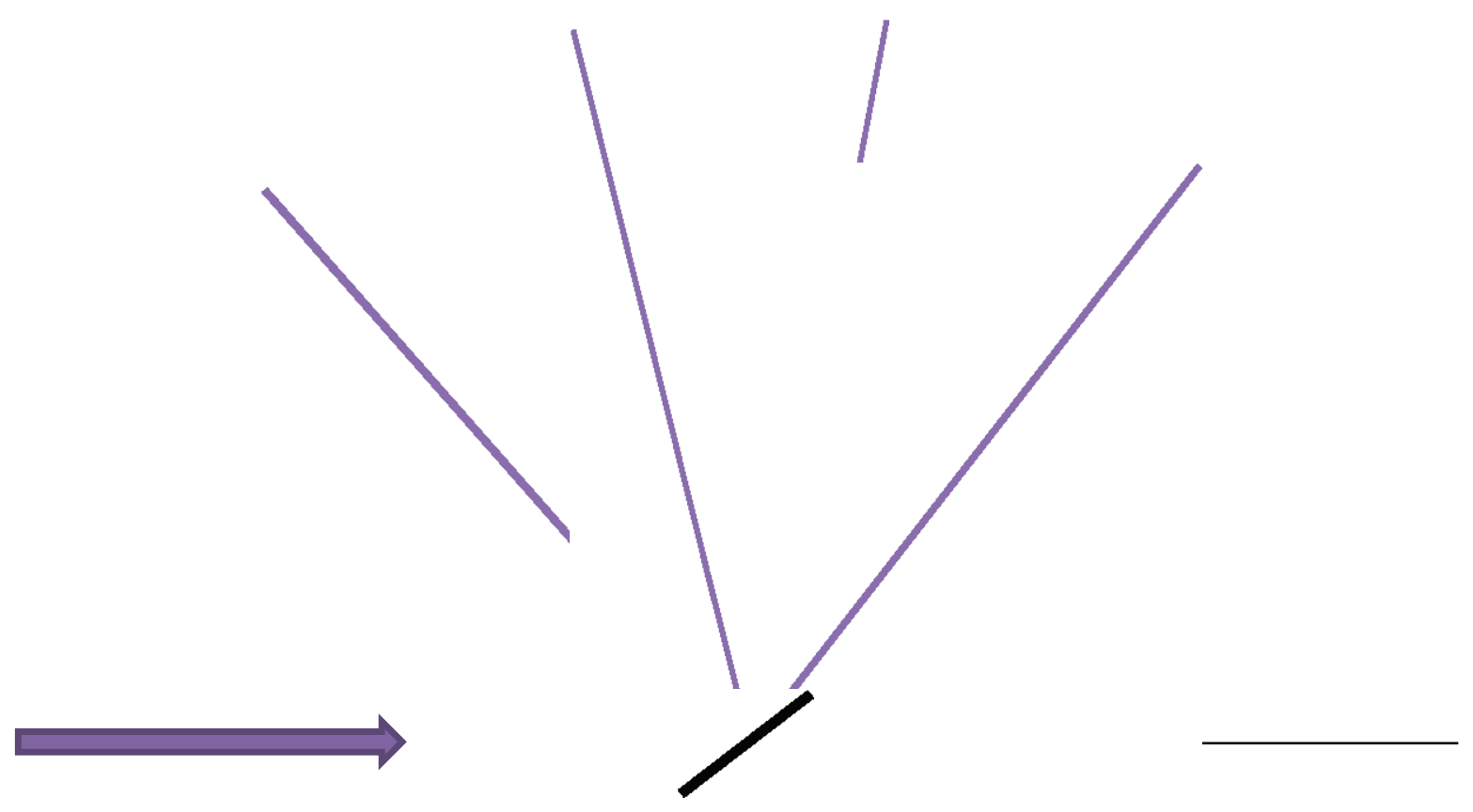

457.9nm 